\title{
Synthesis of Functionalized Diarylmethanes via Copper-Catalyzed Cross-Coupling of Arylmagnesium Reagents with Benzylic Phosphates
}

\author{
Christiane C. Kofink, Paul Knochel* \\ Department Chemie und Biochemie, Ludwig-Maximilians-Universität, Butenandtstrasse 5-13, 81377, München \\ (Germany). \\ Paul.Knochel@cup.uni-muenchen.de
}

\section{Supporting Information}

\section{General considerations}

Unless otherwise indicated, all reactions were carried out with magnetic stirring and in case of air- or moisture-sensitive compounds in flame-dried glassware under nitrogen. Syringes were used to transfer the reagents and the solvents were purged with nitrogen prior to use. Reactions were monitored by gas chromatography (GC and GC-MS) or thin layer chromatography.

Solutions of organomagnesium compounds were prepared, if not especially mentioned, by the reaction of $\mathrm{Mg}$ with aryl bromides in THF, titrated with a standard solution of $\mathrm{I}_{2}$ in $0.5 \mathrm{M} \mathrm{LiCl}$ in THF and diluted with THF to the mentioned concentration. 


\section{Typical Procedures for the Preparation of Functionalized Diarylmethanes:}

\section{Typical Procedure 1 (TP 1).}

In a flame dried Schlenk-tube under nitrogen the Grignard reagent (1.5 equiv.) in DME is cooled to $-15{ }^{\circ} \mathrm{C}$ and $\mathrm{CuCl}(0.1$ equiv. $)$ is added. After 5 min at $-15{ }^{\circ} \mathrm{C}$ triethylphosphite ( 0.2 equiv.) is added and the mixture again stirred for $5 \mathrm{~min}$. Meanwhile a second flame dried schlenk tube is prepared, containing the benzylic phosphate (1.0 equiv.) in DME and tetrabutylammonium iodide ( 0.1 equiv.). This mixture is heated to $60{ }^{\circ} \mathrm{C}$ and then the mixture of the Grignard reagent, $\mathrm{CuCl}$ and triethylphosphite is added via canula over $30 \mathrm{~min}$ to the hot mixture of the benzylic phosphate and tetrabutylammonium iodide. The reaction mixture is stirred at $60{ }^{\circ} \mathrm{C}$ until the $\mathrm{GC}$ of a hydrolyzed aliquot shows completion of the reaction, then quenched with sat. $\mathrm{NH}_{4} \mathrm{Cl}$ solution, extracted with ether, and the product is purified by column chromatography.

\section{Typical Procedure 2 (TP 2).}

In a flame dried Schlenk-tube under nitrogen an aromatic bromide or iodide (1.5. equiv.) is dissolved in DME and cooled to the mentioned temperature. Then $i \mathrm{PrMgCl}$ or $i \mathrm{PrMgCl} \cdot \mathrm{LiCl}$ (1.6 equiv.) is added dropwise via syringe. After completion of the exchange, the Grignard reagent (1.5 equiv.) in $\mathrm{DME}$ is cooled to $-15{ }^{\circ} \mathrm{C}$ and $\mathrm{CuCl}(0.1$ equiv.) is added. After $5 \mathrm{~min}$ at $-15^{\circ} \mathrm{C}$ triethylphosphite ( 0.2 equiv.) is added and the mixture again stirred for 5 min. Meanwhile a second flame dried schlenk tube is prepared, containing the benzylic phosphate (1.0 equiv.) in DME and tetrabutylammonium iodide (0.1 equiv.). This mixture is heated to $60{ }^{\circ} \mathrm{C}$ and then the mixture of the Grignard reagent, $\mathrm{CuCl}$ and triethylphosphite is added via canula over $30 \mathrm{~min}$ to the hot mixture of the benzylic phosphate and tetrabutylammonium iodide. The reaction mixture is stirred at $60{ }^{\circ} \mathrm{C}$ until the $\mathrm{GC}$ of a hydrolyzed aliquot shows completion of the reaction, then quenched with sat. $\mathrm{NH}_{4} \mathrm{Cl}$ solution, extracted with ether, and the product is purified by column chromatography. 


\section{Ethyl 4-benzylbenzoate (2a)}<smiles>CCOC(=O)c1ccc(Cc2ccccc2)cc1</smiles>

Prepared according to the TP 2. To a solution of ethyl 4-iodobenzoate $(207 \mathrm{mg}, 0.75 \mathrm{mmol})$ in $0.5 \mathrm{~mL}$ DME, $i \operatorname{PrMgCl}(0.89 \mathrm{~mL}, 0.80 \mathrm{mmol}, 0.9 \mathrm{M}$ in THF $)$ is added dropwise via syringe at $-20{ }^{\circ} \mathrm{C}$. The solution is stirred for $20 \mathrm{~min}$, than $\mathrm{CuCl}(5.0 \mathrm{mg}, 10 \mathrm{~mol} \%)$ and $\mathrm{P}(\mathrm{OEt})_{3}(17 \mathrm{mg}$, $20 \mathrm{~mol} \%$ ) is added. This mixture is added over $30 \mathrm{~min}$ via canula to a prewarmed mixture of benzyl diethyl phosphate (122 mg, $0.50 \mathrm{mmol})$ and TBAI (19 $\mathrm{mg}, 10 \mathrm{~mol} \%)$. The reaction mixture is heated at $60^{\circ} \mathrm{C}$ for $1 \mathrm{~h}$. The usual workup and purification by flash chromatography (pentane/ether 19:1) yielded 2a as colourless liquid (96 mg, 80\%).

${ }^{1} \mathbf{H}-\mathbf{N M R}\left(\mathrm{CDCl}_{3}, 300 \mathrm{MHz}, 25^{\circ} \mathrm{C}\right): \delta=7.87(\mathrm{~d}, J=8.4 \mathrm{~Hz}, 2 \mathrm{H}), 7.22-7.06(\mathrm{~m}, 7 \mathrm{H}), 4.26(\mathrm{q}$, $J=7.2 \mathrm{~Hz}, 2 \mathrm{H}), 3.92(\mathrm{~s}, 2 \mathrm{H}), 1.28(\mathrm{t}, J=7.1 \mathrm{~Hz}, 3 \mathrm{H})$.

${ }^{13} \mathrm{C}-\mathrm{NMR}\left(\mathrm{CDCl}_{3}, 75 \mathrm{MHz}, 25{ }^{\circ} \mathrm{C}\right): \delta=166.5,146.3,140.1,129.7,128.9,128.5,128.4$, $126.3,60.7,41.9,14.3$.

IR (KBr): 2982 (w), 1711 (vs), 1610 (w), 1602 (w), 1495 (w), 1270 (vs), 1176 (m), 1098 (s), $1020(\mathrm{~m}), 854(\mathrm{w}), 739(\mathrm{~m}), 695(\mathrm{~m})$.

MS (70 eV , EI), m/z (\%): 240 (60, M+), 195 (100), 167 (86), 152 (21), 128 (3), 91 (7), 82 (5).

HRMS $m / z$ : calcd. for $\mathrm{C}_{16} \mathrm{H}_{16} \mathrm{O}_{2}: 240.1150$; found: 240.1141 . 


\section{Ethyl 4-benzylbenzoate (2a)}

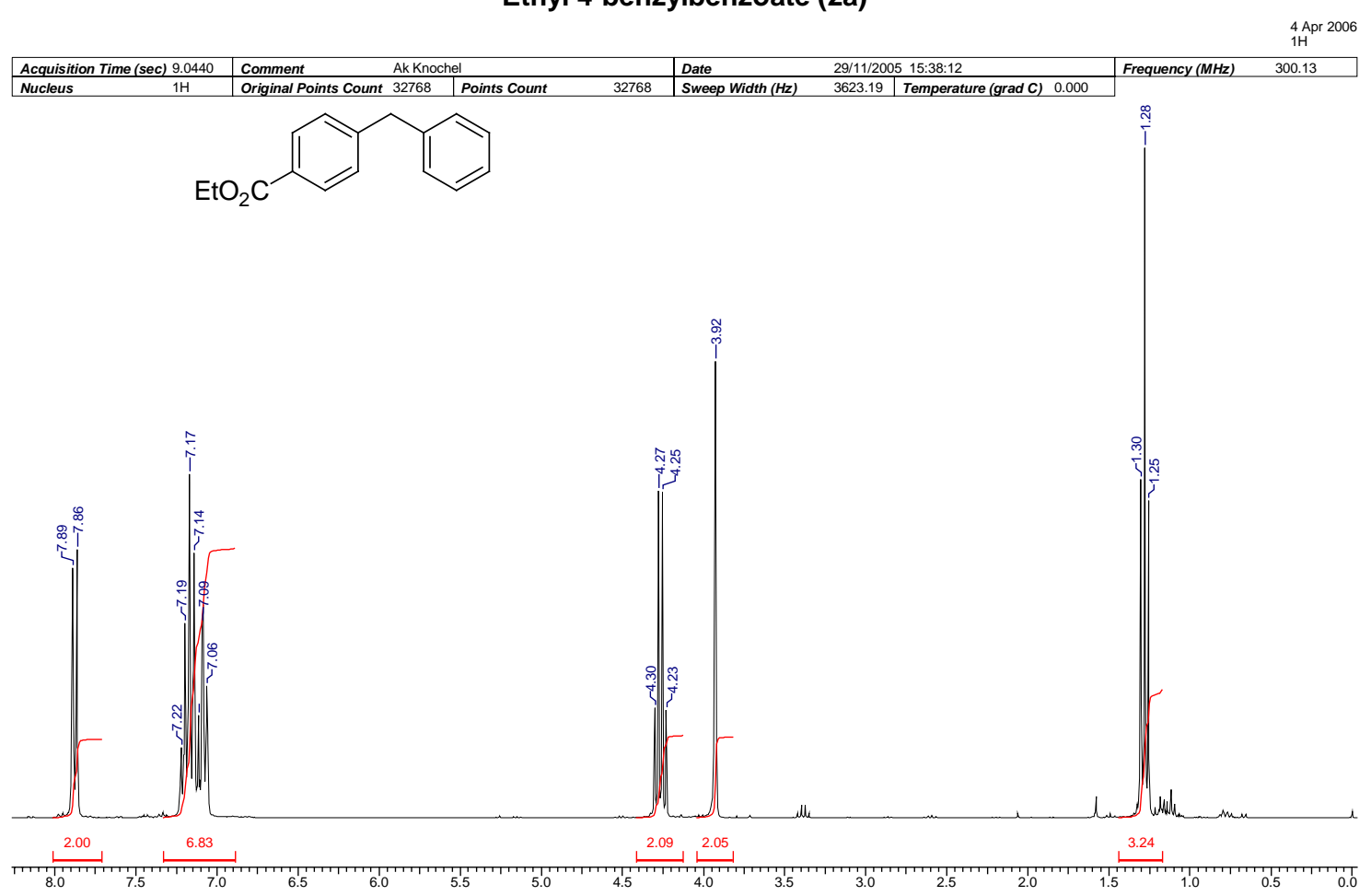

\section{Ethyl 4-benzylbenzoate (2a)}

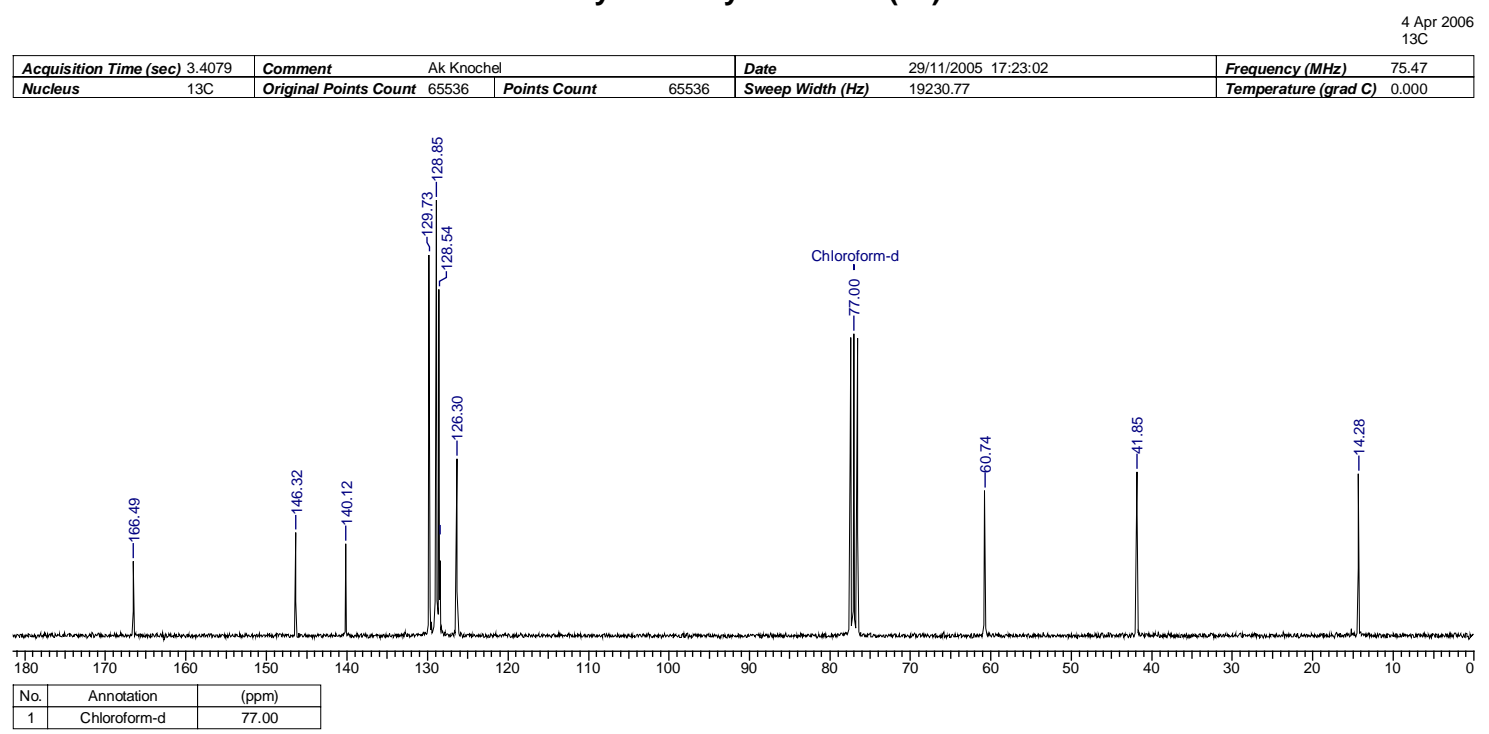




\section{Ethyl 4-(4-methylbenzyl)benzoate (2b)}

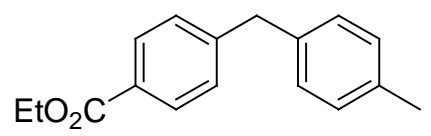

Prepared according to the TP 2. To a solution of ethyl 4-iodobenzoate $(207 \mathrm{mg}, 0.75 \mathrm{mmol})$ in $0.5 \mathrm{~mL}$ DME, $i \operatorname{PrMgCl}(0.89 \mathrm{~mL}, 0.80 \mathrm{mmol}, 0.9 \mathrm{M}$ in THF $)$ is added dropwise via syringe at $-20{ }^{\circ} \mathrm{C}$. The solution is stirred for $20 \mathrm{~min}$, than $\mathrm{CuCl}(5.0 \mathrm{mg}, 10 \mathrm{~mol} \%)$ and $\mathrm{P}(\mathrm{OEt})_{3}(17 \mathrm{mg}$, $20 \mathrm{~mol} \%$ ) is added. This mixture is added over $30 \mathrm{~min}$ via canula to a prewarmed mixture of diethyl 4-methylbenzyl phosphate (129 mg, $0.50 \mathrm{mmol})$ and TBAI (19 mg, $10 \mathrm{~mol} \%)$. The reaction mixture is heated at $60{ }^{\circ} \mathrm{C}$ for $1 \mathrm{~h}$. The usual workup and purification by flash chromatography (pentane/ether 19:1) yielded $\mathbf{2 b}$ as colourless liquid (112 $\mathrm{mg}, 88 \%$ ).

${ }^{1} \mathbf{H}$-NMR $\left(\mathrm{CDCl}_{3}, 300 \mathrm{MHz}, 25{ }^{\circ} \mathrm{C}\right): \delta=7.86(\mathrm{~d}, J=8.4 \mathrm{~Hz}, 2 \mathrm{H}), 7.14(\mathrm{~d}, J=8.5 \mathrm{~Hz}, 2 \mathrm{H})$, 7.01-6.94 (m, 4H), 4.25 (q, J=7.2 Hz, 2H), $3.87(\mathrm{~s}, 2 \mathrm{H}), 3.08(\mathrm{~s}, 3 \mathrm{H}), 1.27$ (t, J= $7.1 \mathrm{~Hz}, 3 \mathrm{H})$. ${ }^{13} \mathrm{C}-\mathrm{NMR}\left(\mathrm{CDCl}_{3}, 75 \mathrm{MHz}, 25{ }^{\circ} \mathrm{C}\right): \delta=166.5,146.6,140.1,137.1,135.8,129.7,129.2$, $128.8,128.3,60.7,41.4,20.9,14.3$.

IR (KBr): 2981 (w), 1713 (vs), 1609 (m), 1514 (w), 1366 (w), 1271 (vs), 1176 (m), 1103 (s), $1020(\mathrm{~m}), 869(\mathrm{w}), 738(\mathrm{~m})$.

MS (70 eV, EI), m/z (\%): 254 (90, M+ ), 239 (26), 209 (76), 181 (100), 165 (50), 105 (9), 77 (5).

HRMS $m / z$ : calcd. for $\mathrm{C}_{17} \mathrm{H}_{18} \mathrm{O}_{2}: 254.1307$; found: 254.1306 . 
Ethyl 4-(4-methylbenzyl)benzoate (2b)

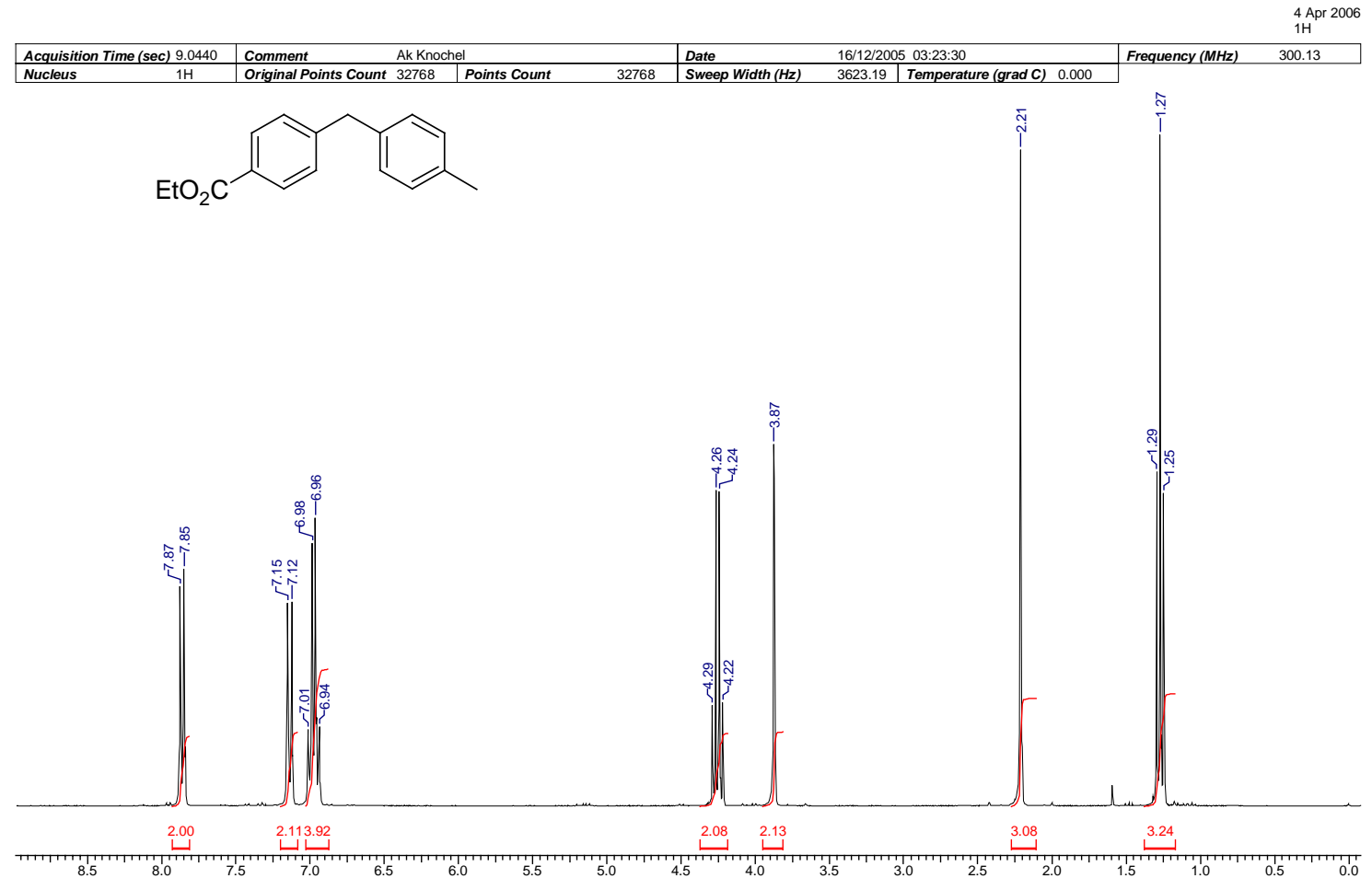

Ethyl 4-(4-methylbenzyl)benzoate (2b)

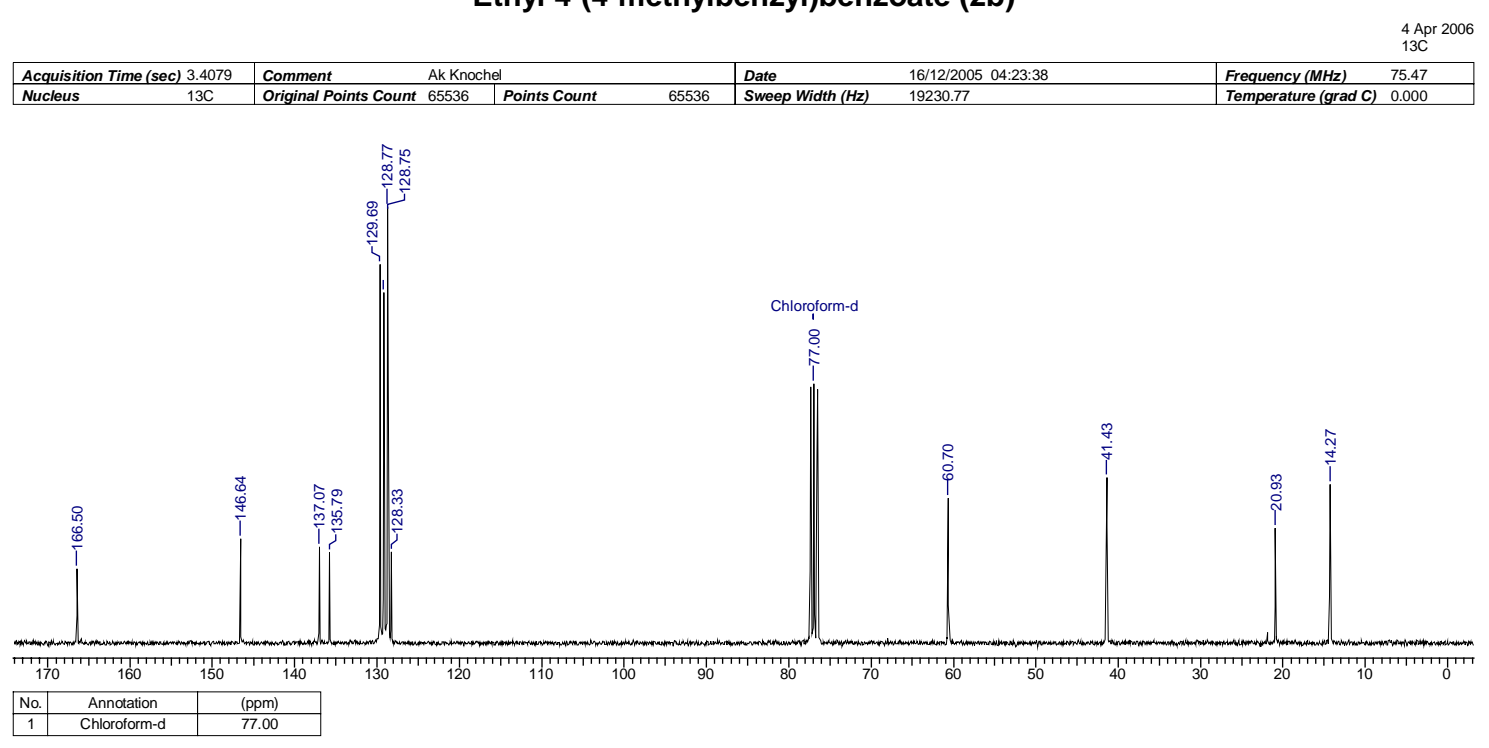




\section{Ethyl 4-(4-bromobenzyl)benzoate (2c)}

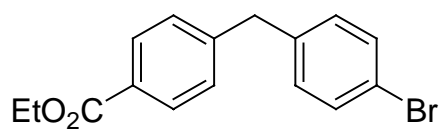

Prepared according to the TP 2. To a solution of ethyl 4-iodobenzoate(207 $\mathrm{mg}, 0.75 \mathrm{mmol})$ in $0.5 \mathrm{~mL}$ DME, $i \mathrm{PrMgCl}(0.89 \mathrm{~mL}, 0.80 \mathrm{mmol}, 0.9 \mathrm{M}$ in THF $)$ is added dropwise via syringe at $-20{ }^{\circ} \mathrm{C}$. The solution is stirred for $20 \mathrm{~min}$, than $\mathrm{CuCl}(5.0 \mathrm{mg}, 10 \mathrm{~mol} \%)$ and $\mathrm{P}(\mathrm{OEt})_{3}(17 \mathrm{mg}$, $20 \mathrm{~mol} \%$ ) is added. This mixture is added over $30 \mathrm{~min}$ via canula to a prewarmed mixture of 4-bromobenzyl diethyl phosphate (162 mg, $0.50 \mathrm{mmol})$ and TBAI (19 mg, $10 \mathrm{~mol} \%)$. The reaction mixture is heated at $60{ }^{\circ} \mathrm{C}$ for $1 \mathrm{~h}$. The usual workup and purification by flash chromatography (pentane/ether 9:1) yielded 2c as colourless liquid (115 mg, 72\%).

${ }^{1} \mathbf{H}$-NMR $\left(\mathrm{CDCl}_{3}, 300 \mathrm{MHz}, 25{ }^{\circ} \mathrm{C}\right): \delta=7.83(\mathrm{~d}, J=8.2 \mathrm{~Hz}, 2 \mathrm{H}), 7.31(\mathrm{~d}, J=8.2 \mathrm{~Hz}, 2 \mathrm{H})$, $7.12(\mathrm{~d}, J=8.2 \mathrm{~Hz}, 2 \mathrm{H}), 6.94(\mathrm{~d}, J=8.2 \mathrm{~Hz}, 2 \mathrm{H}), 4.27(\mathrm{q}, J=7.1 \mathrm{~Hz}, 2 \mathrm{H}), 3.87(\mathrm{~s}, 2 \mathrm{H}), 1.28$ (t, $J=7.1 \mathrm{~Hz}, 3 \mathrm{H})$.

${ }^{13} \mathrm{C}-\mathrm{NMR}\left(\mathrm{CDCl}_{3}, 75 \mathrm{MHz}, 25{ }^{\circ} \mathrm{C}\right): \delta=166.4,145.6,139.1,131.6,130.6,129.8,128.8$, 128.7, 120.2, 60.8, 41.2, 14.3 .

IR (KBr): 2981 (w), 1712 (vs), 1610 (m), 1487 (m), 1403 (w), 1271 (vs), 1176 (m), 1102 (s), $1020(\mathrm{~m}), 101(\mathrm{~s}), 927(\mathrm{w}), 754(\mathrm{~m})$.

MS (70 eV, EI), m/z (\%): 318 (74, M+), 290 (17), 275 (90), 245 (50), 211 (20), 165 (100), 97 (11), $82(10)$.

HRMS $m / z$ : calcd. for $\mathrm{C}_{16} \mathrm{H}_{15} \mathrm{BrO}_{2}: 318.0255$; found: 318.0242 . 
Ethyl 4-(4-bromobenzyl)benzoate (2c)

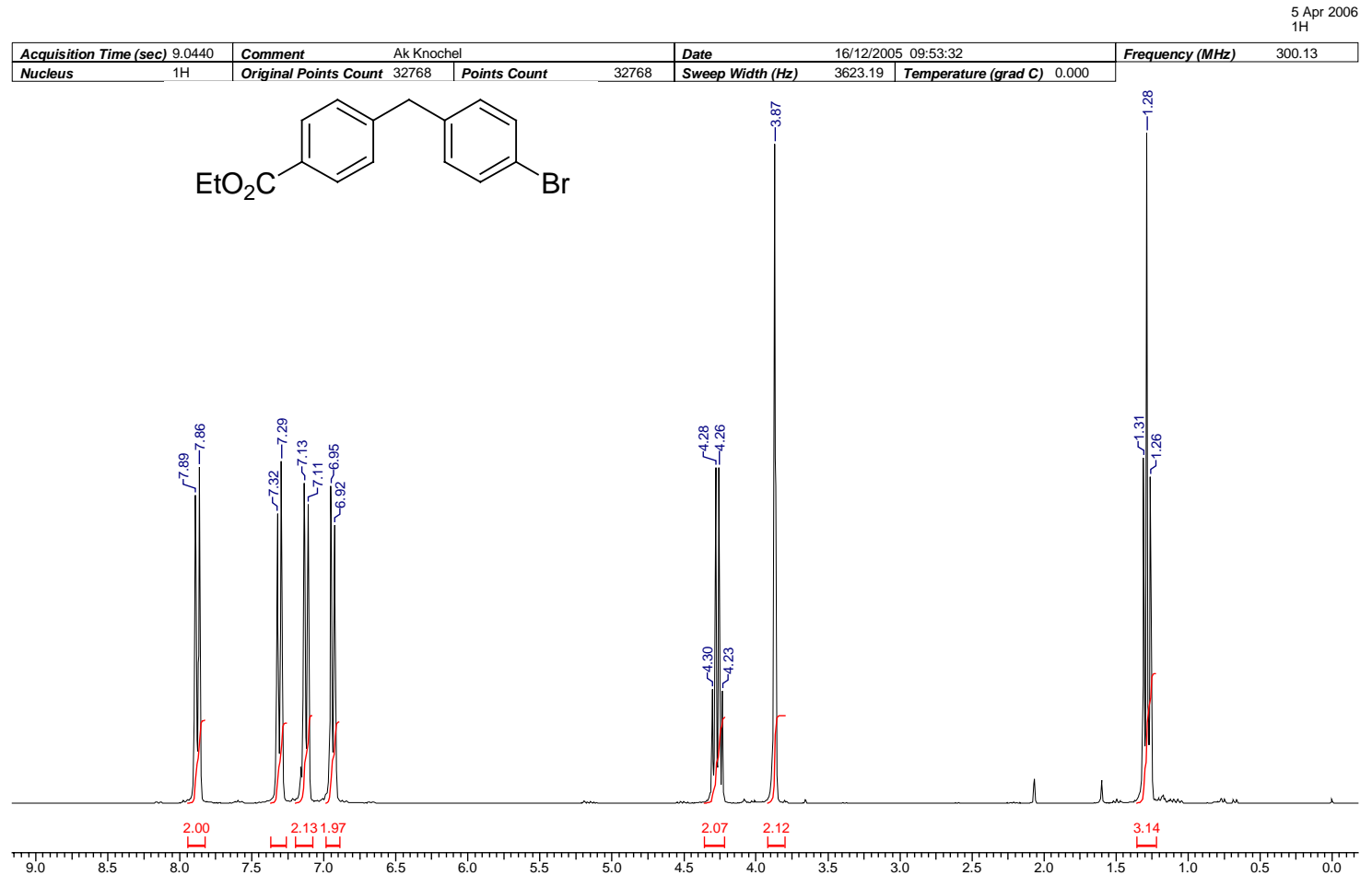

\section{Ethyl 4-(4-bromobenzyl)benzoate (2c)}
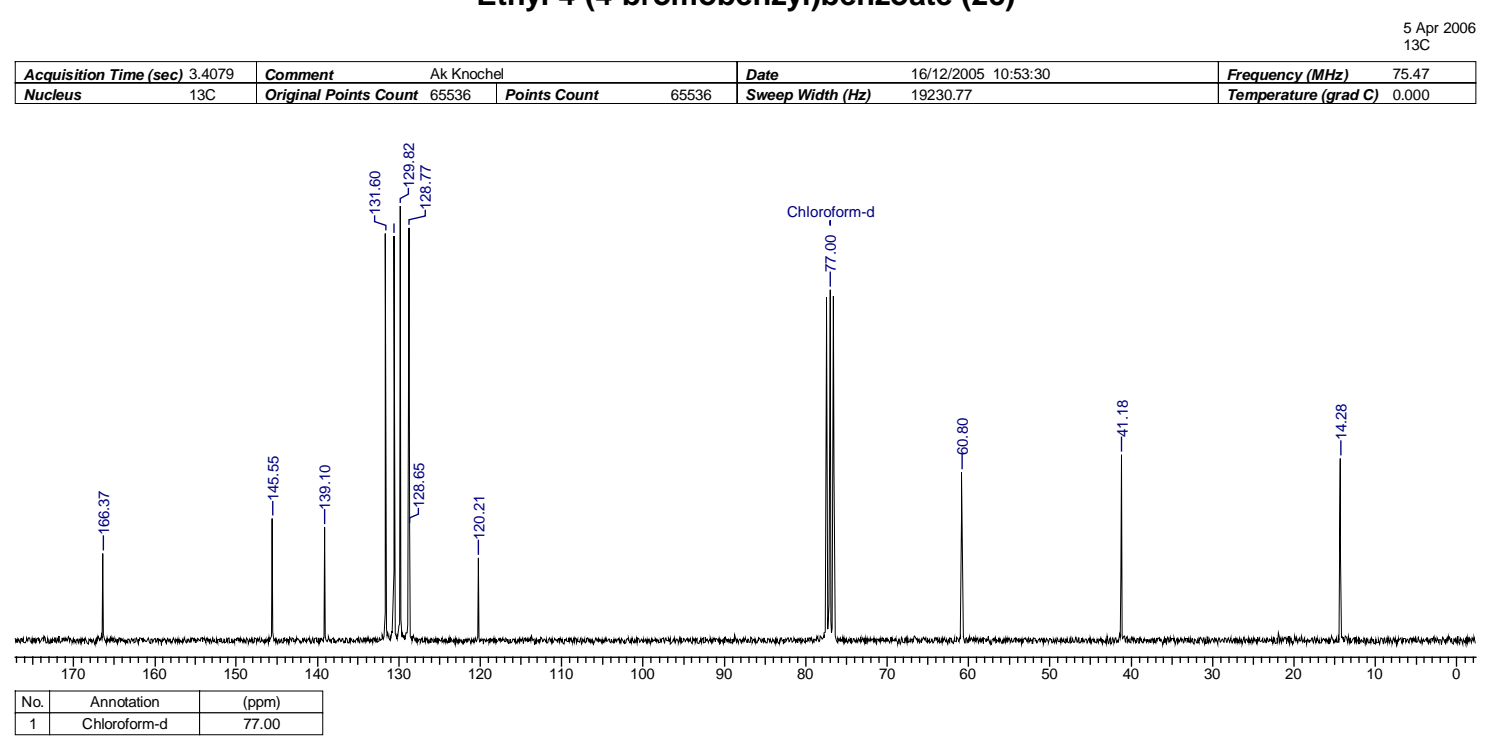


\section{Ethyl 4-(2-methoxybenzyl)benzoate (2d)}

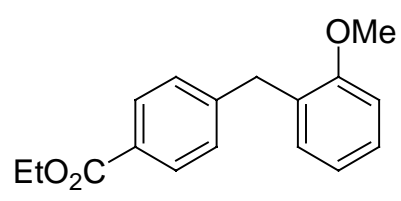

Prepared according to the TP 2. To a solution of ethyl 4-iodobenzoate $(207 \mathrm{mg}, 0.75 \mathrm{mmol})$ in $0.5 \mathrm{~mL}$ DME, $i \mathrm{PrMgCl}(0.89 \mathrm{~mL}, 0.80 \mathrm{mmol}, 0.9 \mathrm{M}$ in THF $)$ is added dropwise via syringe at $-20{ }^{\circ} \mathrm{C}$. The solution is stirred for $20 \mathrm{~min}$, than $\mathrm{CuCl}(5.0 \mathrm{mg}, 10 \mathrm{~mol} \%)$ and $\mathrm{P}(\mathrm{OEt})_{3}(17 \mathrm{mg}$, $20 \mathrm{~mol} \%$ ) is added. This mixture is added over $30 \mathrm{~min}$ via canula to a prewarmed mixture of diethyl 2-methoxybenzyl phosphate (137 mg, $0.50 \mathrm{mmol})$ and TBAI (19 $\mathrm{mg}, 10 \mathrm{~mol} \%)$. The reaction mixture is heated at $60{ }^{\circ} \mathrm{C}$ for $1 \mathrm{~h}$. The usual workup and purification by flash chromatography (pentane/ether 9:1) yielded 2d as colourless liquid (109 mg, 81\%).

${ }^{1}$ H-NMR $\left(\mathrm{CDCl}_{3}, 300 \mathrm{MHz}, 25^{\circ} \mathrm{C}\right): \delta=7.85(\mathrm{~d}, J=8.3 \mathrm{~Hz}, 2 \mathrm{H}), 7.17-7.07(\mathrm{~m}, 3 \mathrm{H}), 6.96(\mathrm{~d}$, $J=7.2 \mathrm{~Hz}, 1 \mathrm{H}), 6.80-6.74(\mathrm{~m}, 2 \mathrm{H}), 4.24(\mathrm{q}, J=7.1 \mathrm{~Hz}, 2 \mathrm{H}), 3.91(\mathrm{~s}, 2 \mathrm{H}), 3.67(\mathrm{~s}, 3 \mathrm{H}), 1.26$ (t, $J=7.2 \mathrm{~Hz}, 3 \mathrm{H})$.

${ }^{13} \mathrm{C}-\mathrm{NMR}\left(\mathrm{CDCl}_{3}, 75 \mathrm{MHz}, 25{ }^{\circ} \mathrm{C}\right): \delta=166.6,157.3,146.5,130.3,129.5,128.8,128.6$, $128.1,127.7,120.5,110.4,60.6,55.2,36.0,14.3$.

IR (KBr): 2981 (w), 1172 (vs), 1610 (m), 1492 (m), 1415 (w), 1271 (vs), 1242 (vs), 1176 (m), 1103 (s), 1020 (s), 928 (w), 747 (s), 701 (w).

MS (70 eV, EI), m/z (\%): 270 (100, M+), 225 (45), 197 (77), 165 (25), 152 (11), 91 (39).

HRMS $m / z$ : calcd. for $\mathrm{C}_{17} \mathrm{H}_{18} \mathrm{O}_{3}: 270.1256$; found: 270.1257 . 
Ethyl 4-(2-methoxybenzyl)benzoate (2d)

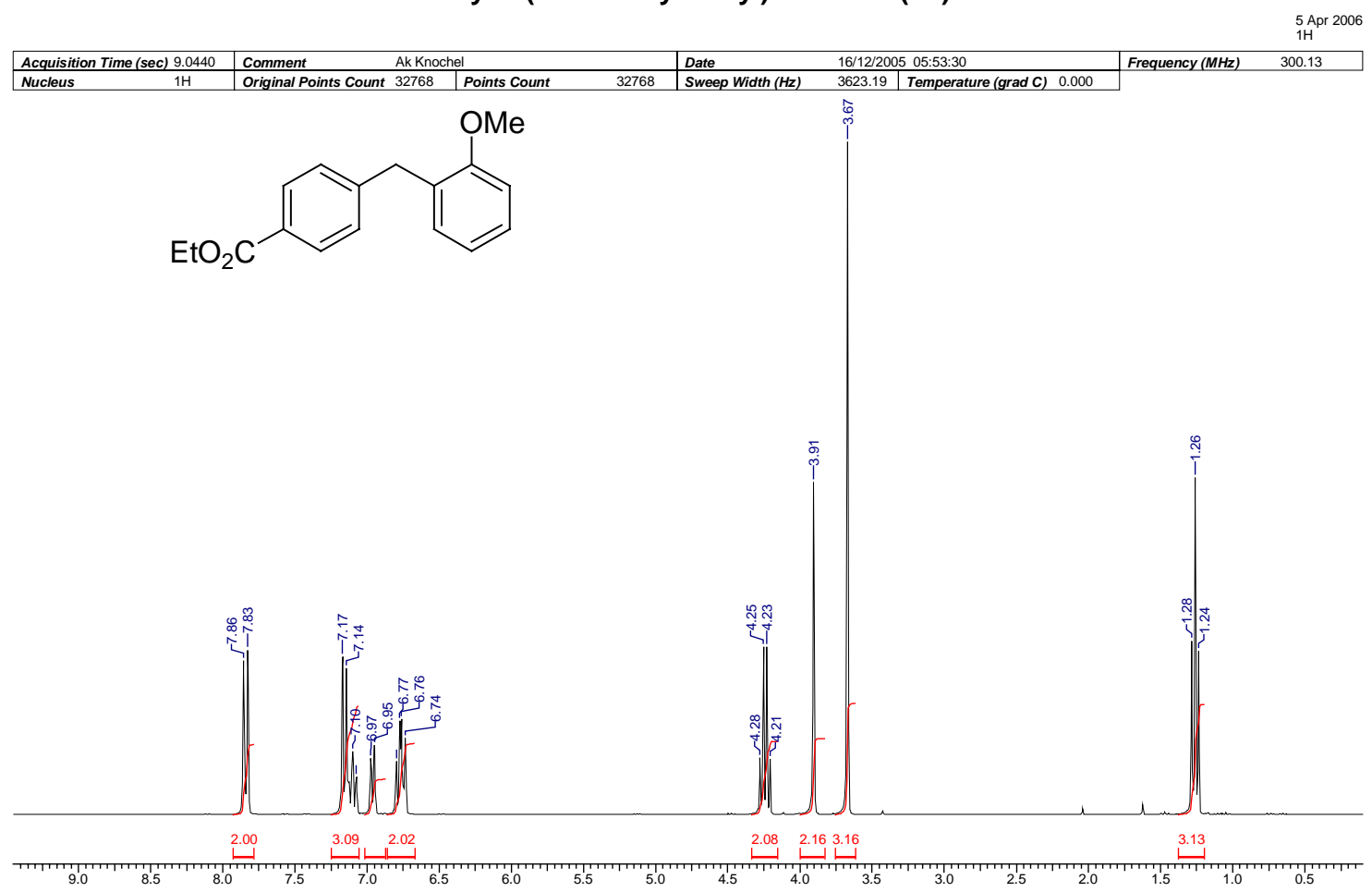

Ethyl 4-(2-methoxybenzyl)benzoate (2d)

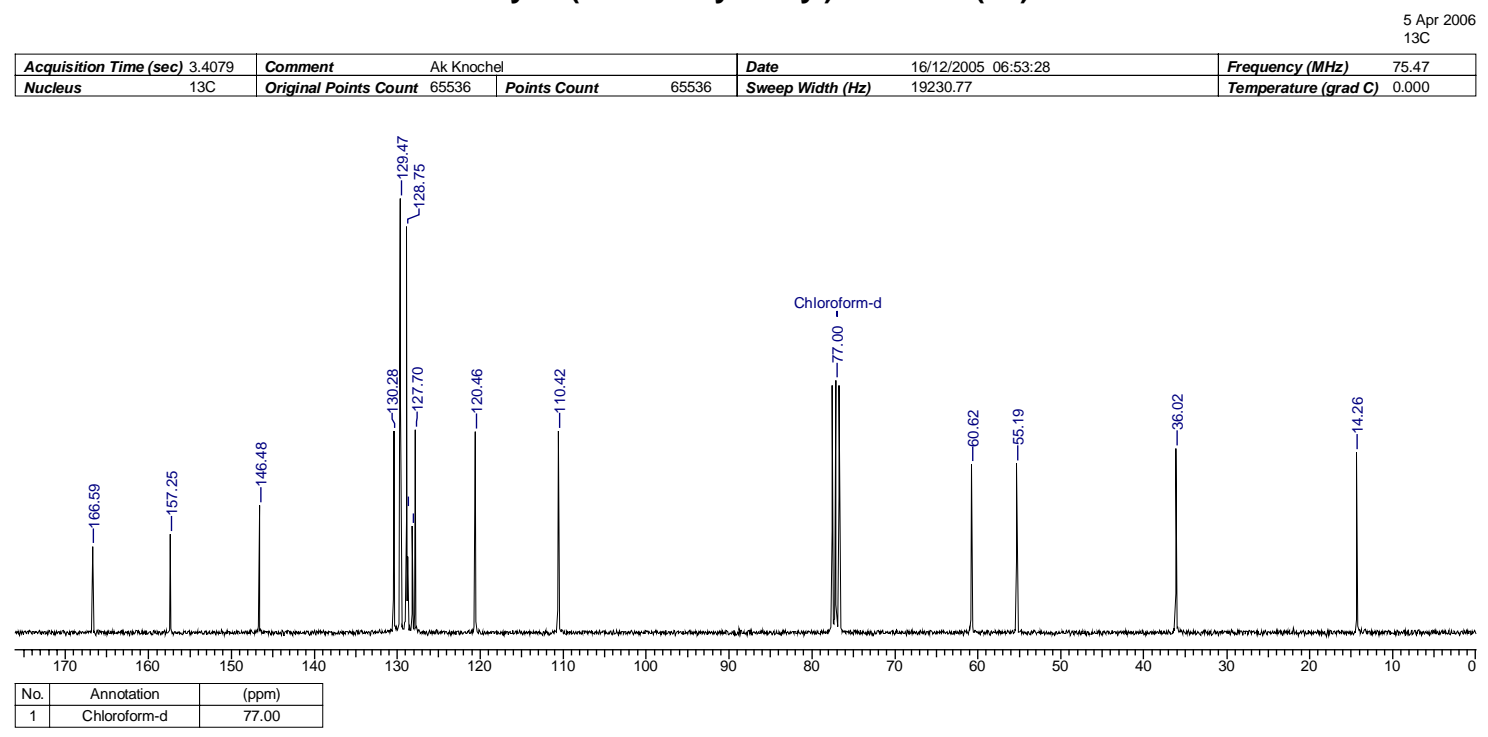




\section{1-Benzyl-4-methoxybenzene (2e)}

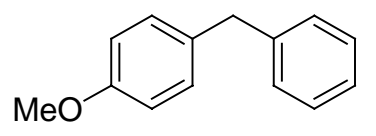

Prepared according to the TP 1. To a solution of 4-methoxyphenylmagnesium bromide $\left(0.87 \mathrm{~mL}, 0.75 \mathrm{mmol}, 0.86 \mathrm{M}\right.$ in THF) in $0.5 \mathrm{~mL} \mathrm{DME}, \mathrm{CuCl}(5.0 \mathrm{mg}, 10 \mathrm{~mol} \%)$ and $\mathrm{P}(\mathrm{OEt})_{3}$ $(17 \mathrm{mg}, 20 \mathrm{~mol} \%)$ is added. This mixture is added over $30 \mathrm{~min}$ via canula to a prewarmed mixture of benzyl diethyl phosphate $(122 \mathrm{mg}, 0.50 \mathrm{mmol})$ and TBAI (19 $\mathrm{mg}, 10 \mathrm{~mol} \%)$. The reaction mixture is heated at $60{ }^{\circ} \mathrm{C}$ for $1 \mathrm{~h}$. The usual workup and purification by flash chromatography (pentane) yielded $2 \mathrm{e}$ as colourless liquid (72 $\mathrm{mg}, 73 \%)$.

${ }^{1}$ H-NMR $\left(\mathrm{CDCl}_{3}, 300 \mathrm{MHz}, 25^{\circ} \mathrm{C}\right): \delta=7.21-6.99(\mathrm{~m}, 7 \mathrm{H}), 6.73(\mathrm{~d}, J=8.7 \mathrm{~Hz}, 2 \mathrm{H}), 3.83(\mathrm{~s}$, 2H), 3.67 (s, 3H).

${ }^{13} \mathrm{C}-\mathrm{NMR}\left(\mathrm{CDCl}_{3}, 75 \mathrm{MHz}, 25{ }^{\circ} \mathrm{C}\right): \delta=158.0,141.6,133.2,129.8,128.8,128.4,125.9$, 133.9, 55.2, 41.0 .

IR (KBr): 3062 (w), 3027 (w), 1610 (m), 1510 (vs), 1494 (m), 1440 (m), 1300 (w), 1243 (vs), 1175 (m), 1034 (m), 836 (w), 768 (w), 723 (m), 696 (m).

MS (70 eV, EI), m/z (\%): 198 (100, M+), 167 (30), 121 (22), 91 (8), 51 (4).

HRMS $m / z$ : calcd. for $\mathrm{C}_{14} \mathrm{H}_{14} \mathrm{O}: 198.1045$; found: 198.1033 . 


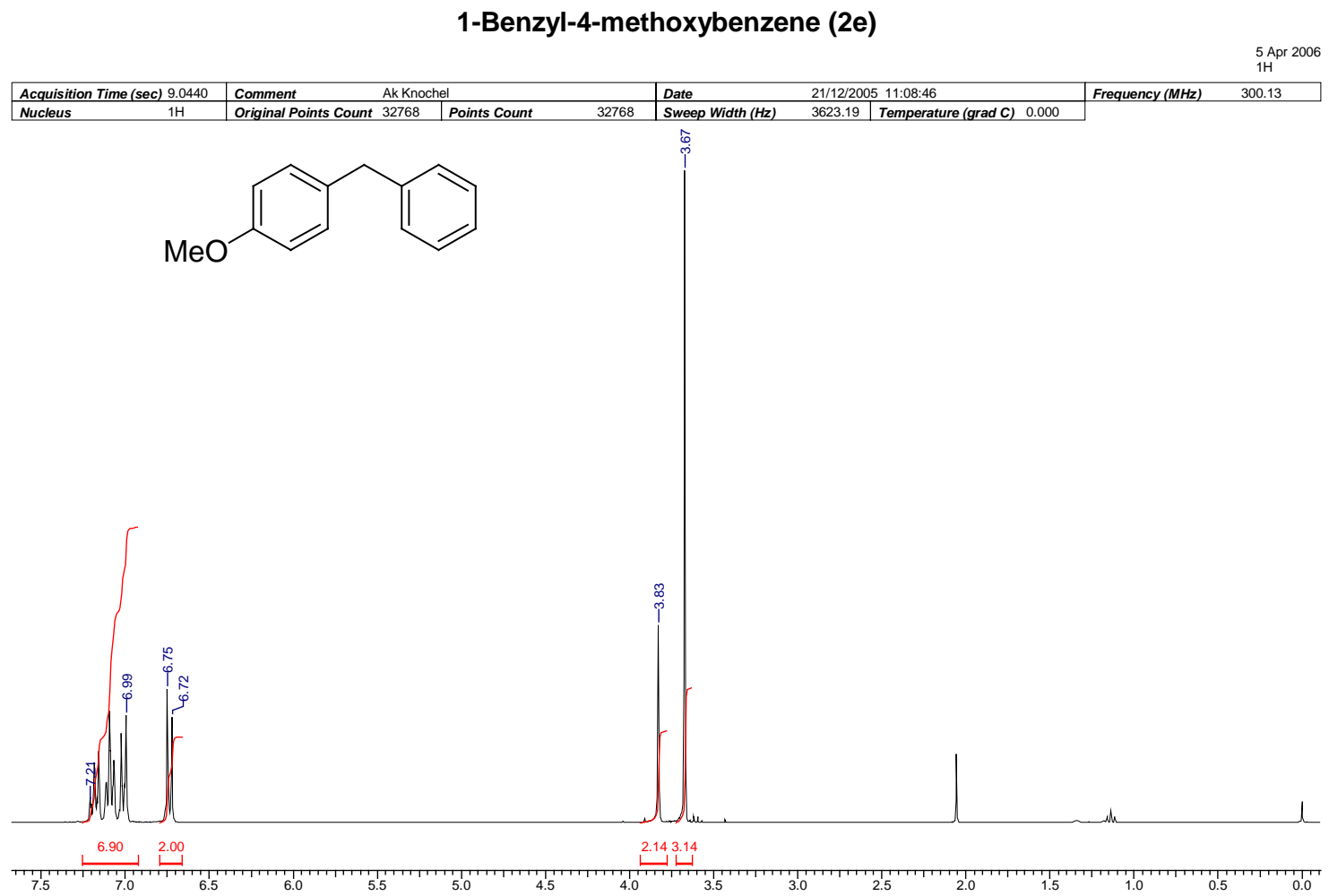

1-Benzyl-4-methoxybenzene (2e)

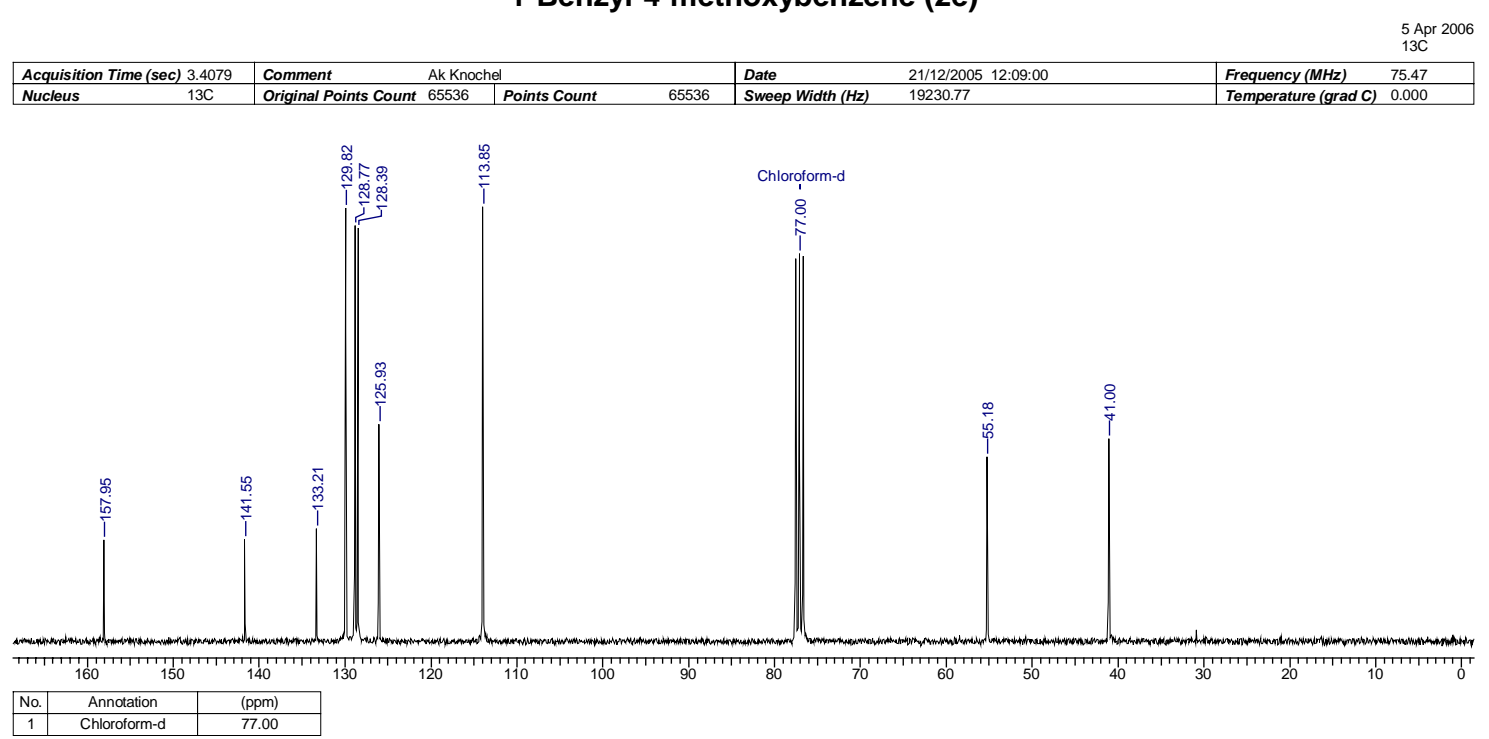




\section{1-Methoxy-4-(4-methylbenzyl)benzene (2f)}

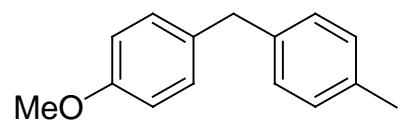

Prepared according to the TP 1. To a solution of 4-methoxyphenylmagnesium bromide $\left(0.87 \mathrm{~mL}, 0.75 \mathrm{mmol}, 0.86 \mathrm{M}\right.$ in THF) in $0.5 \mathrm{~mL} \mathrm{DME}, \mathrm{CuCl}(5.0 \mathrm{mg}, 10 \mathrm{~mol} \%)$ and $\mathrm{P}(\mathrm{OEt})_{3}$ (17 $\mathrm{mg}, 20 \mathrm{~mol} \%$ ) is added. This mixture is added over $30 \mathrm{~min}$ via canula to a prewarmed mixture of diethyl 4-methylbenzyl phosphate $(129 \mathrm{mg}, 0.50 \mathrm{mmol})$ and TBAI $(19 \mathrm{mg}$, $10 \mathrm{~mol} \%$ ). The reaction mixture is heated at $60^{\circ} \mathrm{C}$ for $1 \mathrm{~h}$. The usual workup and purification by flash chromatography (pentane) yielded $\mathbf{2 f}$ as colourless liquid (85 $\mathrm{mg}, 80 \%)$.

${ }^{1} \mathbf{H}$-NMR $\left(\mathrm{CDCl}_{3}, 300 \mathrm{MHz}, 2{ }^{\circ} \mathrm{C}\right): \delta=7.02-6.96(\mathrm{~m}, 6 \mathrm{H}), 6.74(\mathrm{~d}, J=8.6 \mathrm{~Hz}, 2 \mathrm{H}), 3.80(\mathrm{~s}$, 2H), 3.69 (s, 3H), 2.23 (s, 3H).

${ }^{13} \mathbf{C}$-NMR $\left(\mathrm{CDCl}_{3}, 75 \mathrm{MHz}, 25{ }^{\circ} \mathrm{C}\right): \delta=157.9,138.5,135.4,133.5,129.8,129.1,128.7$, $133.8,55.2,40.6,21.0$.

IR (KBr): 3002 (w), 2909 (w), 1610 (w), 1508 (vs), 1462 (m), 1301 (w), 1242 (vs), 1175 (m), $1106(\mathrm{w}), 1036(\mathrm{~m}), 848(\mathrm{w}), 804(\mathrm{~m})$.

MS (70 eV, EI), m/z (\%): 212 (100, M+), 197 (76), 181 (20), 165 (15), 121 (14), 105 (8), 77 (6).

HRMS $m / z$ : calcd. for $\mathrm{C}_{15} \mathrm{H}_{16} \mathrm{O}: 212.1201$; found: 212.1215 . 
1-Methoxy-4-(4-methylbenzyl)benzene (2f)

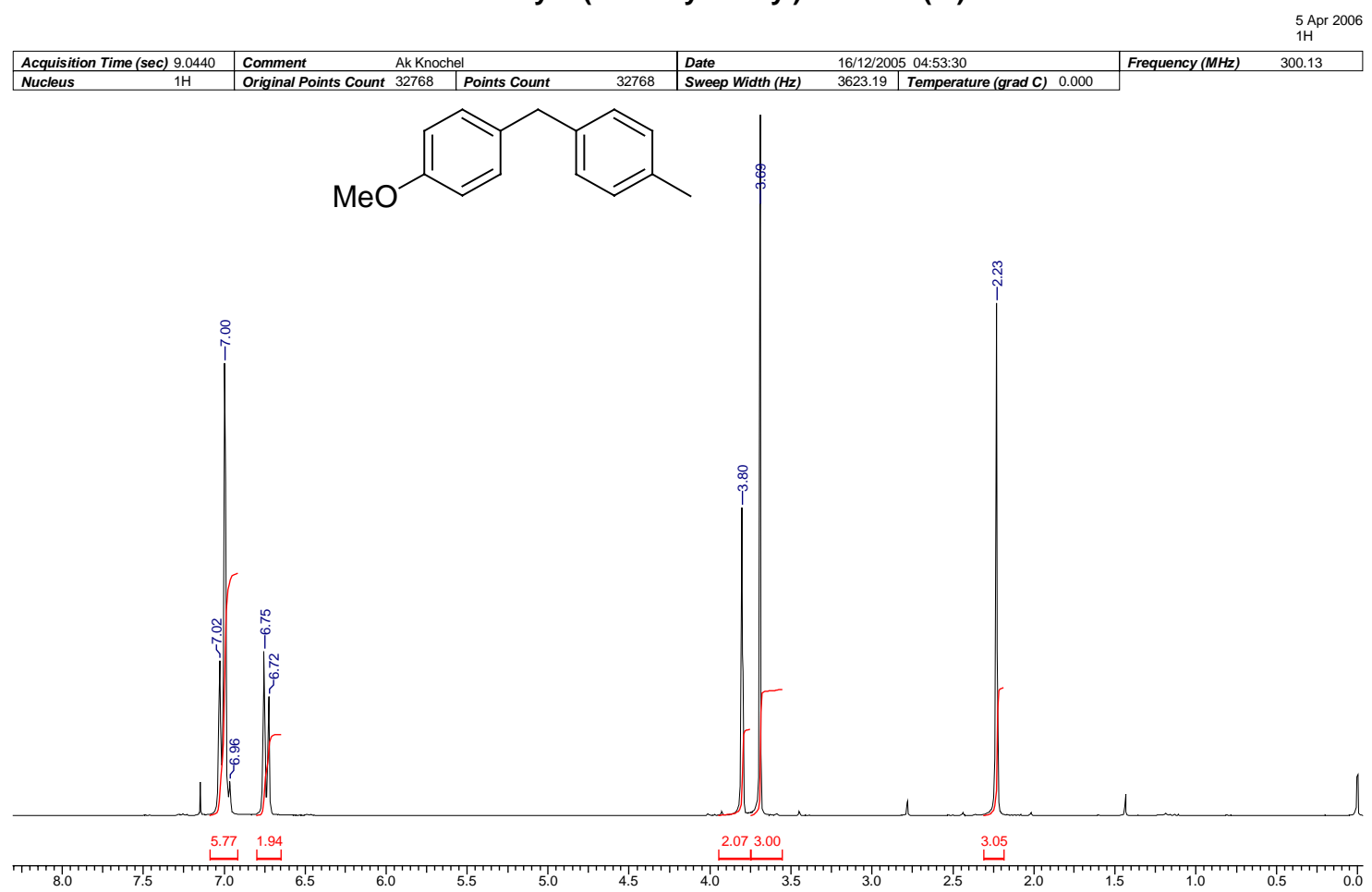

\section{1-Methoxy-4-(4-methylbenzyl)benzene (2f)}

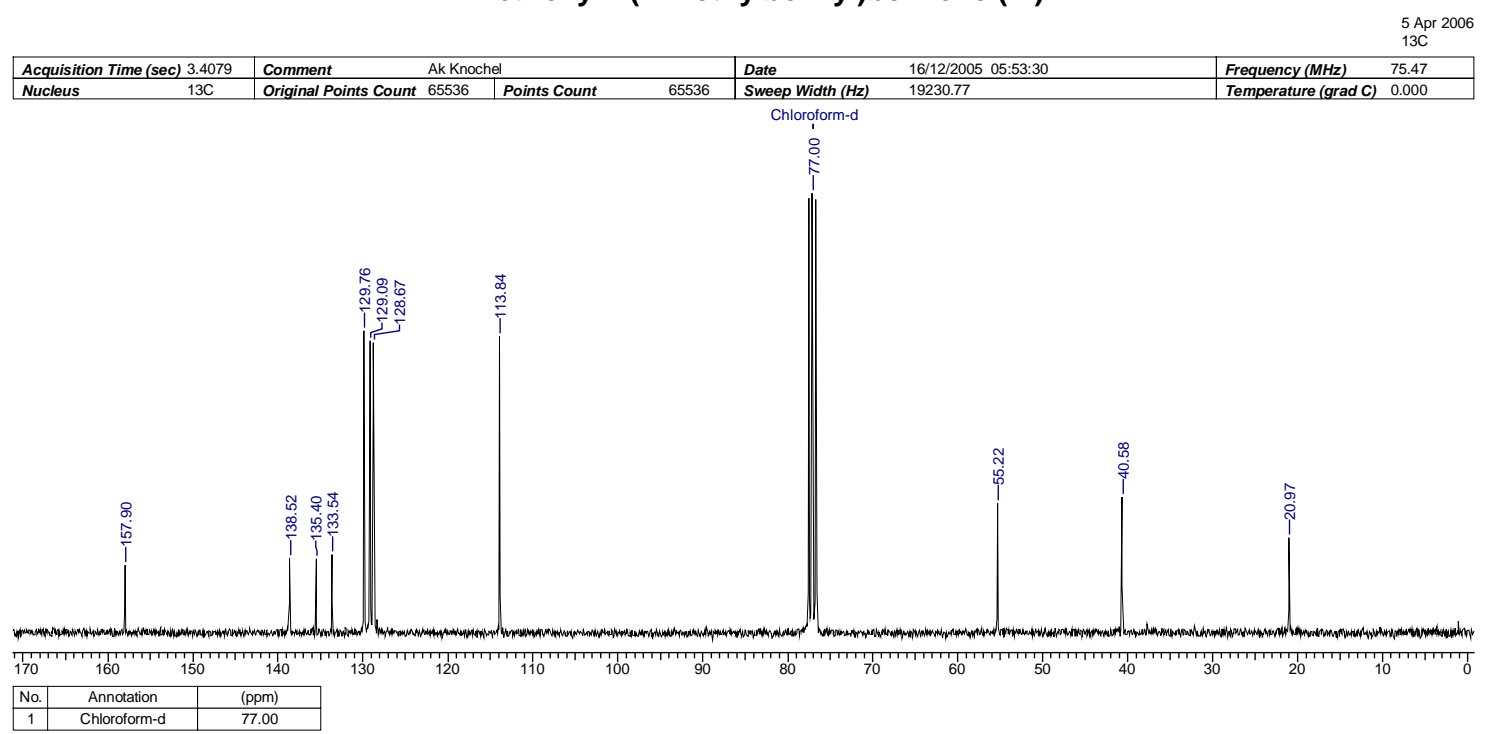




\section{4-(4-Methoxybenzyl)benzonitrile (2g)}

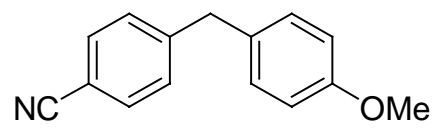

Prepared according to the TP 2. To a solution of 4-bromobenzonitrile $(137 \mathrm{mg}, 0.75 \mathrm{mmol})$ in $0.5 \mathrm{~mL}$ DME, $i \mathrm{PrMgCl} \cdot \mathrm{LiCl}(0.73 \mathrm{~mL}, 0.80 \mathrm{mmol}, 1.1 \mathrm{M}$ in THF $)$ is added dropwise via syringe at $0{ }^{\circ} \mathrm{C}$. The solution is stirred for $3 \mathrm{~h}$, than $\mathrm{CuCl}(5.0 \mathrm{mg}, 10 \mathrm{~mol} \%)$ and $\mathrm{P}(\mathrm{OEt})_{3}$ (17 mg, $20 \mathrm{~mol} \%$ ) is added. This mixture is added over $30 \mathrm{~min}$ via canula to a prewarmed mixture of diethyl 4-methoxybenzyl phosphate $(137 \mathrm{mg}, 0.50 \mathrm{mmol})$ and TBAI $(19 \mathrm{mg}$, $10 \mathrm{~mol} \%$ ). The reaction mixture is heated at $60^{\circ} \mathrm{C}$ for $1 \mathrm{~h}$. The usual workup and purification by flash chromatography (pentane/ether 9:1) yielded $\mathbf{2 g}$ as colourless liquid (80 $\mathrm{mg}, 72 \%$ ).

${ }^{1}$ H-NMR $\left(\mathrm{CDCl}_{3}, 300 \mathrm{MHz}, 25{ }^{\circ} \mathrm{C}\right): \delta=7.45(\mathrm{~d}, J=8.5 \mathrm{~Hz}, 2 \mathrm{H}), 7.17(\mathrm{~d}, J=8.5 \mathrm{~Hz}, 2 \mathrm{H})$, $6.98(\mathrm{~d}, J=8.7 \mathrm{~Hz}, 2 \mathrm{H}), 6.76(\mathrm{~d}, J=8.7 \mathrm{~Hz}, 2 \mathrm{H}), 3.87$ (s, 2H), 3.69 (s, 3H).

${ }^{13} \mathbf{C}$-NMR $\left(\mathrm{CDCl}_{3}, 75 \mathrm{MHz}, 25{ }^{\circ} \mathrm{C}\right): \delta=158.3,147.2,132.2,131.3,129.9,129.4,118.9$, 114.1, 109.8, 55.2, 41.0.

IR (KBr): 2933 (w), 2226 (m), 1609 (m), 1510 (vs), 1462 (m), 1440 (m), 1301 (m), 1244 (vs), 1176 (s), 1110 (m), 1032 (s), 917 (w), 861 (m), 809 (s), 761 (m).

MS (70 eV, EI), m/z (\%): 223 (100, M+), 208 (23), 192 (13), 180 (12), 121 (27), 77(6).

HRMS m/z: calcd. for $\mathrm{C}_{15} \mathrm{H}_{13} \mathrm{NO}: 223.0997$; found: 223.0981 . 

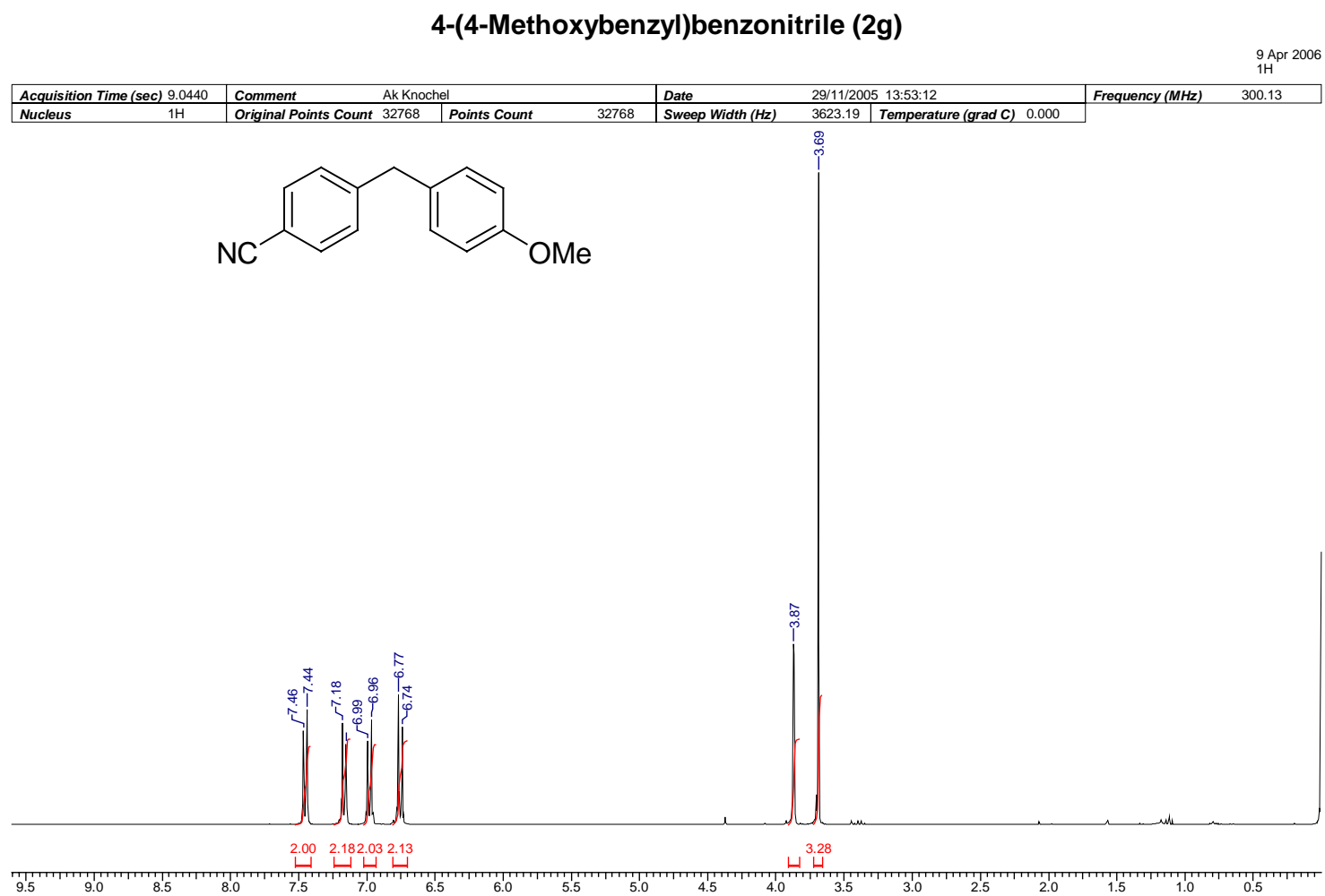

\section{4-(4-Methoxybenzyl)benzonitrile (2g)}
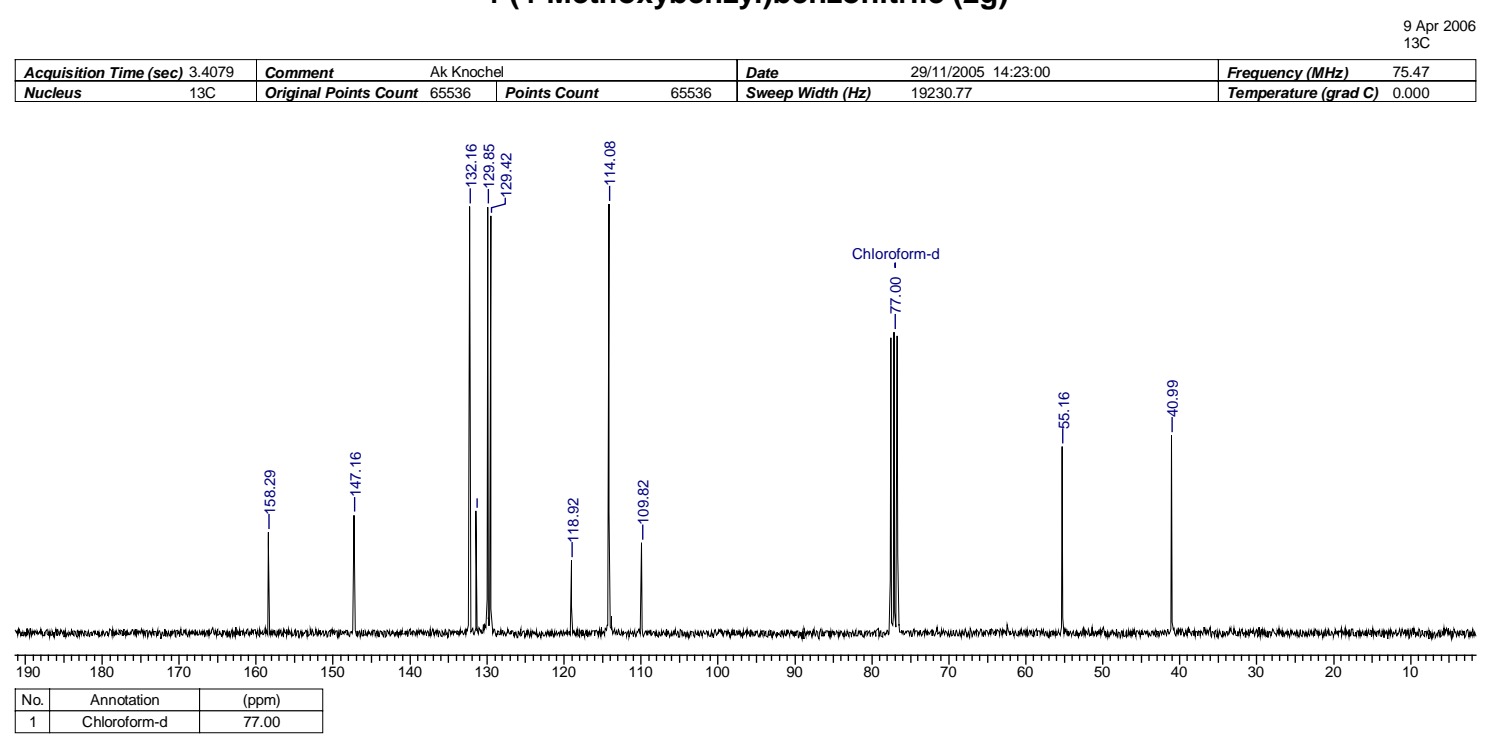


\section{4-(Furan-3-ylmethyl)benzonitrile (2h)}

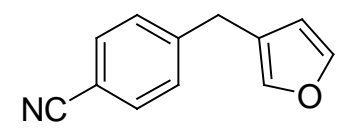

Prepared according to the TP 2. To a solution of 4-bromobenzonitrile $(137 \mathrm{mg}, 0.75 \mathrm{mmol})$ in $0.5 \mathrm{~mL}$ DME, $i \mathrm{PrMgCl} \cdot \mathrm{LiCl}(0.73 \mathrm{~mL}, 0.80 \mathrm{mmol}, 1.1 \mathrm{M}$ in THF $)$ is added dropwise via syringe at $0{ }^{\circ} \mathrm{C}$. The solution is stirred for $3 \mathrm{~h}$, than $\mathrm{CuCl}(5.0 \mathrm{mg}, 10 \mathrm{~mol} \%)$ and $\mathrm{P}(\mathrm{OEt})_{3}$ (17 $\mathrm{mg}, 20 \mathrm{~mol} \%$ ) is added. This mixture is added over $30 \mathrm{~min}$ via canula to a prewarmed mixture of diethyl furan-3-ylmethyl phosphate $(117 \mathrm{mg}, 0.50 \mathrm{mmol})$ and TBAI $(19 \mathrm{mg}$, $10 \mathrm{~mol} \%$ ). The reaction mixture is heated at $60{ }^{\circ} \mathrm{C}$ for $1 \mathrm{~h}$. The usual workup and purification by flash chromatography (pentane/ether $49: 1$ ) yielded $\mathbf{2 h}$ as colourless liquid (60 $\mathrm{mg}, 63 \%$ ).

${ }^{1} \mathbf{H}-\mathbf{N M R}\left(\mathrm{CDCl}_{3}, 300 \mathrm{MHz}, 25^{\circ} \mathrm{C}\right): \delta=7.60(\mathrm{~d}, J=8.1 \mathrm{~Hz}, 2 \mathrm{H}), 7.40$ (s br, 1H), $7.34(\mathrm{~d}, J=$ $8.1 \mathrm{~Hz}, 2 \mathrm{H}), 7.26$ (s br, 1H), 6.22 (s br, 1H), 3.85 (s, 2H).

${ }^{13} \mathrm{C}-\mathrm{NMR}\left(\mathrm{CDCl}_{3}, 75 \mathrm{MHz}, 25{ }^{\circ} \mathrm{C}\right): \delta=145.9,143.4,139.8,132.2,129.3,122.6,118.9$, 110.9, 110.1, 31.2.

IR (KBr): 2920 (w), 2228 (s), 1767 (w), 1712 (w), 1607 (m), 1501 (m), 1416 (m), 1383 (w), 1278 (w), 1175 (m), 1066 (s), 1020 (vs), 975 (s), 915 (m), 873 (s), 786 (vs), 749 (s), 679 (m). MS (70 eV, EI), m/z (\%): $183\left(100, \mathrm{M}^{+}\right), 154$ (57), 127 (27), 115 (10), 51 (5).

HRMS $m / z$ : calcd. for $\mathrm{C}_{12} \mathrm{H}_{9} \mathrm{NO}$ : 183.0684 ; found: 183.0696 . 
4-(Furan-3-yImethyl)benzonitrile (2h)

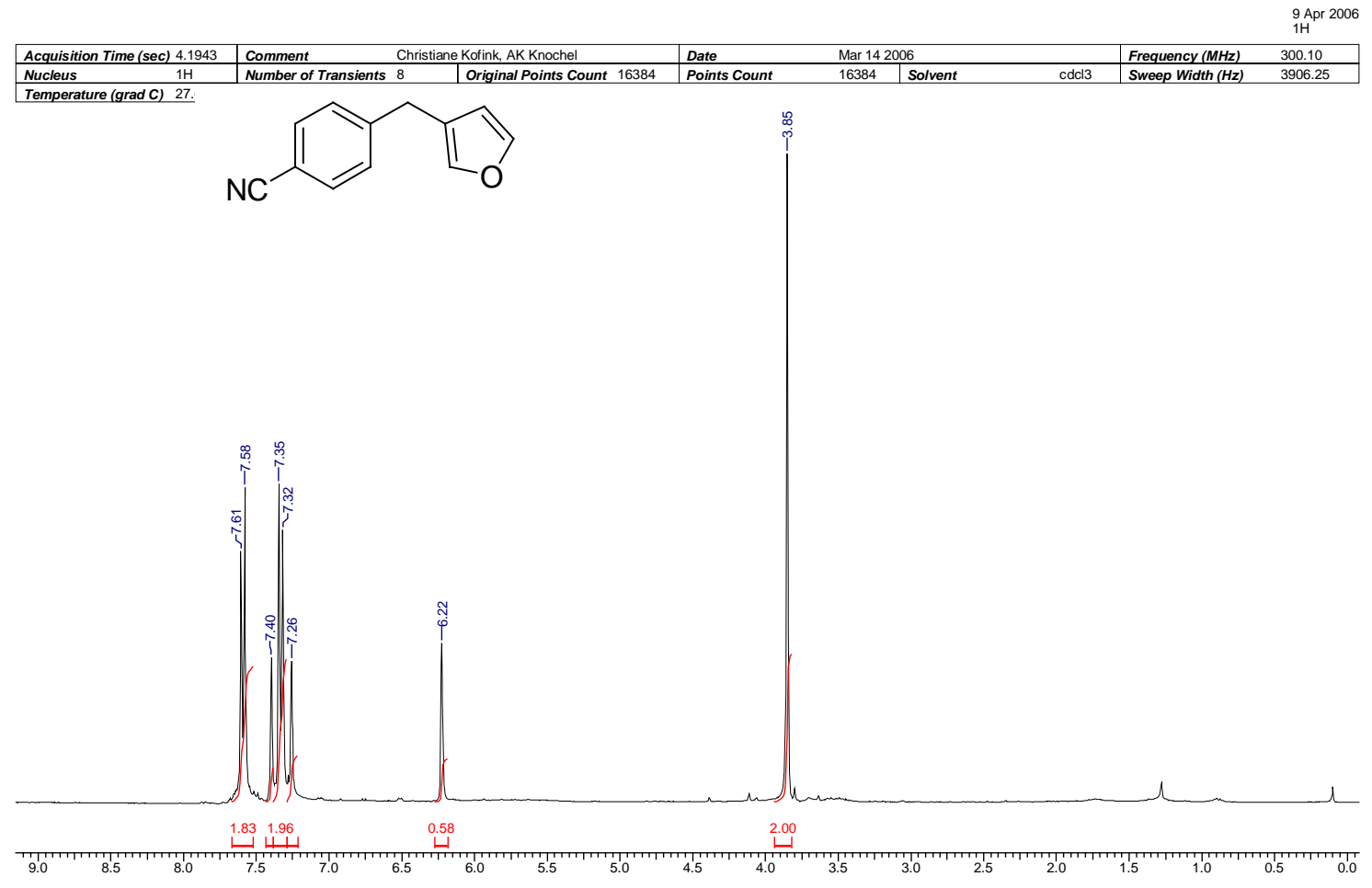

4-(Furan-3-ylmethyl)benzonitrile (2h)
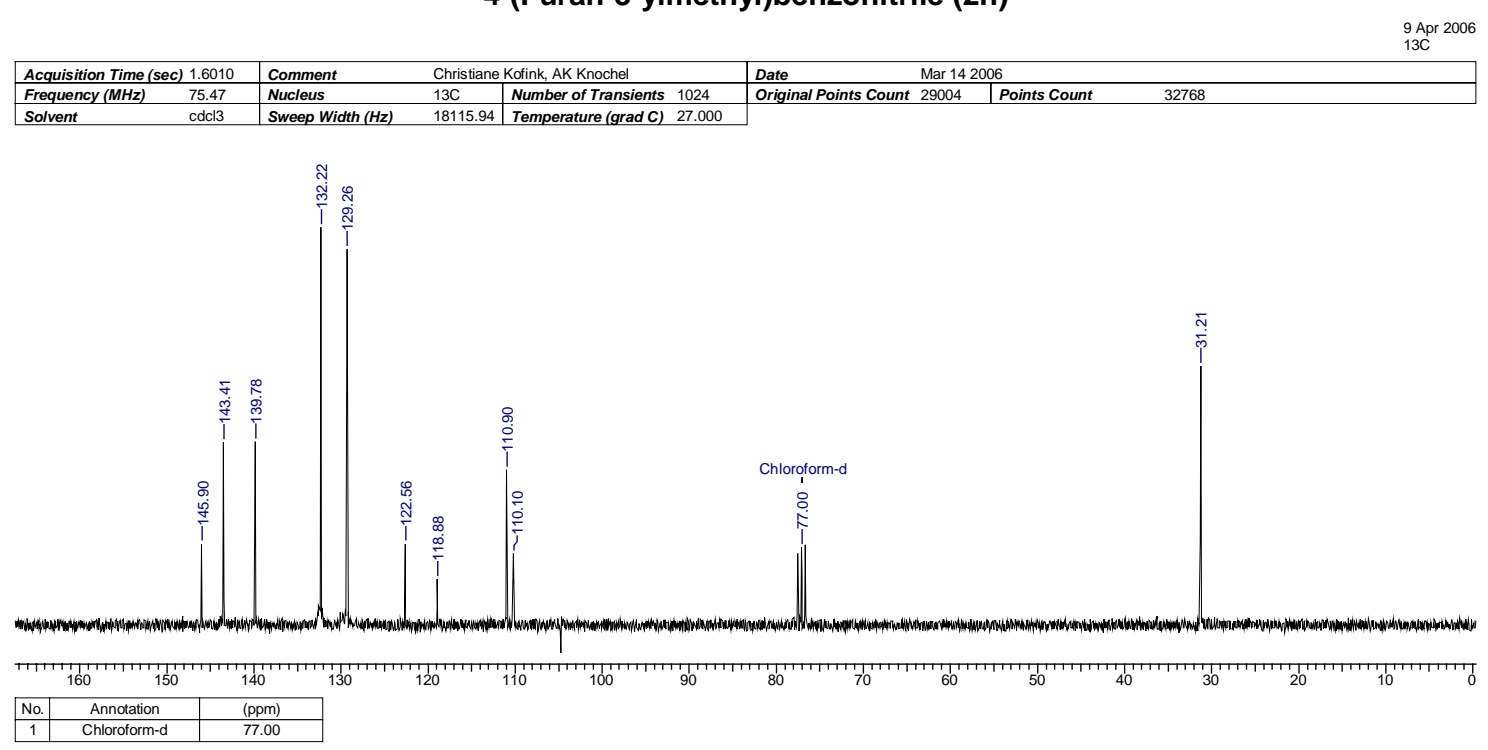


\section{2-(Furan-3-ylmethyl)benzonitrile (2i)}

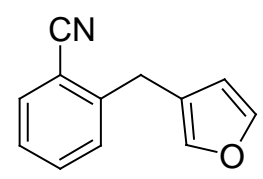

Prepared according to the TP 2. To a solution of 2-bromobenzonitrile (137 $\mathrm{mg}, 0.75 \mathrm{mmol})$ in $0.5 \mathrm{~mL}$ DME, $i \mathrm{PrMgCl} \cdot \mathrm{LiCl}(0.73 \mathrm{~mL}, 0.80 \mathrm{mmol}, 1.1 \mathrm{M}$ in THF $)$ is added dropwise via syringe at $0{ }^{\circ} \mathrm{C}$. The solution is stirred for $3 \mathrm{~h}$, than $\mathrm{CuCl}(5.0 \mathrm{mg}, 10 \mathrm{~mol} \%)$ and $\mathrm{P}(\mathrm{OEt})_{3}$ (17 mg, $20 \mathrm{~mol} \%$ ) is added. This mixture is added over $30 \mathrm{~min}$ via canula to a prewarmed mixture of diethyl furan-3-ylmethyl phosphate $(117 \mathrm{mg}, 0.50 \mathrm{mmol})$ and TBAI (19 mg, $10 \mathrm{~mol} \%$ ). The reaction mixture is heated at $60{ }^{\circ} \mathrm{C}$ for $1 \mathrm{~h}$. The usual workup and purification by flash chromatography (pentane/ether 49:1) yielded $\mathbf{2 i}$ as colourless liquid (60 $\mathrm{mg}, 63 \%$ ).

${ }^{1}$ H-NMR $\left(\mathrm{CDCl}_{3}, 300 \mathrm{MHz}, 25^{\circ} \mathrm{C}\right): \delta=7.51-7.35$ (m, 5H), 7.22 (s br, 1H), 6.19 (s br, 1H), $3.79(\mathrm{~s}, 2 \mathrm{H})$.

${ }^{13} \mathrm{C}-\mathrm{NMR}\left(\mathrm{CDCl}_{3}, 75 \mathrm{MHz}, 25{ }^{\circ} \mathrm{C}\right): \delta=143.5,141.8,139.8,133.1,132.0,130.0129 .2$, $122.7,112.5,110.9,104.7,30.7$.

IR (KBr): 2917 (w), 2229 (s), 17770 (w), 1582 (w), 1430 (w), 1154 (w), 1066 (m), 1022 (s), 976 (m), 873 (s), 782 (s), 734 (s), 710 (m).

MS (70 eV, EI), m/z (\%): $183\left(100, \mathrm{M}^{+}\right), 154$ (63), 127 (26), 115 (9), 81 (11), 51 (6).

HRMS $m / z$ : calcd. for $\mathrm{C}_{12} \mathrm{H}_{9} \mathrm{NO}$ : 183.0684 ; found: 183.0689 . 
2-(Furan-3-ylmethyl)benzonitrile (2i)

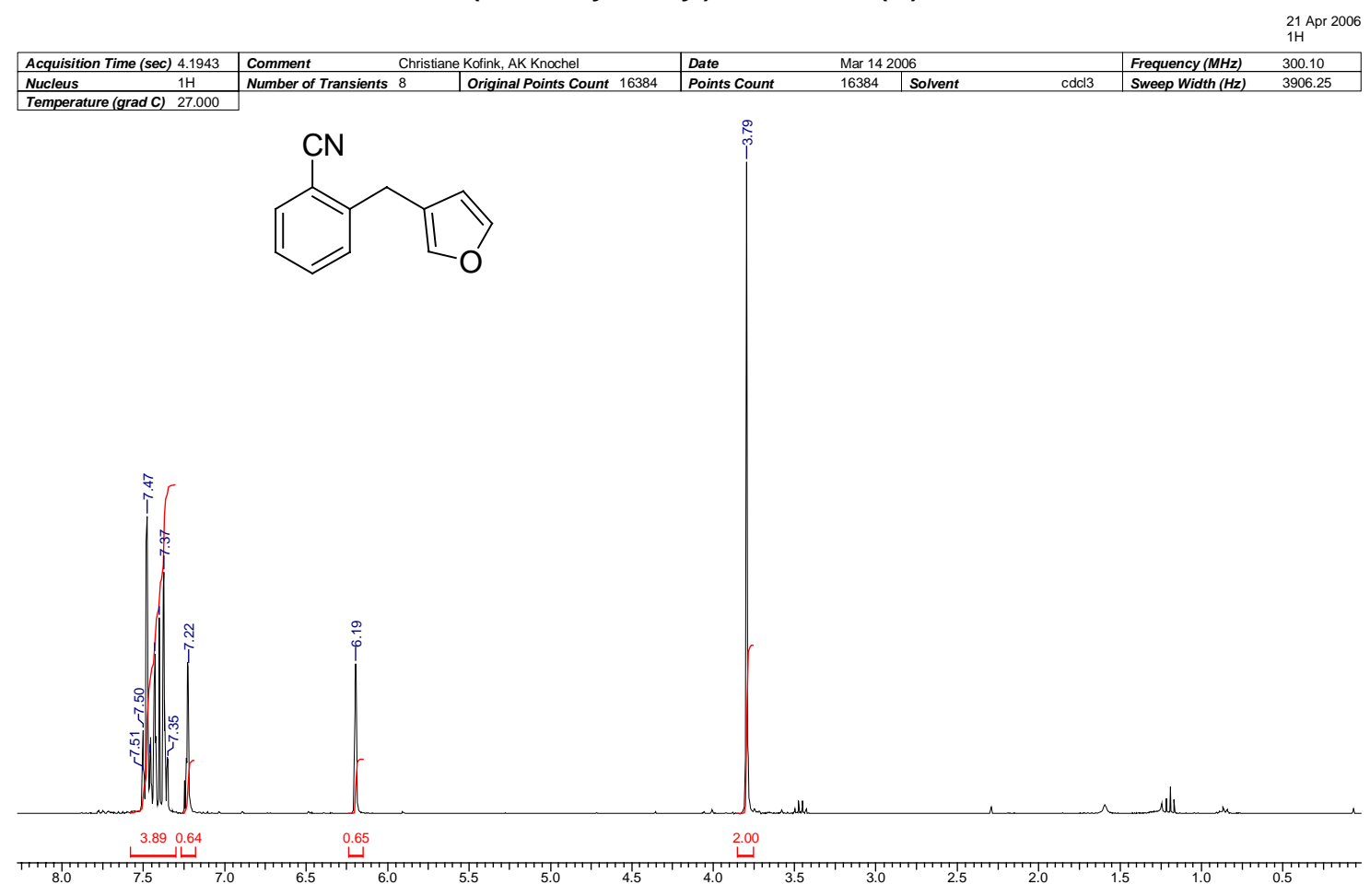

\section{2-(Furan-3-ylmethyl)benzonitrile (2i)}

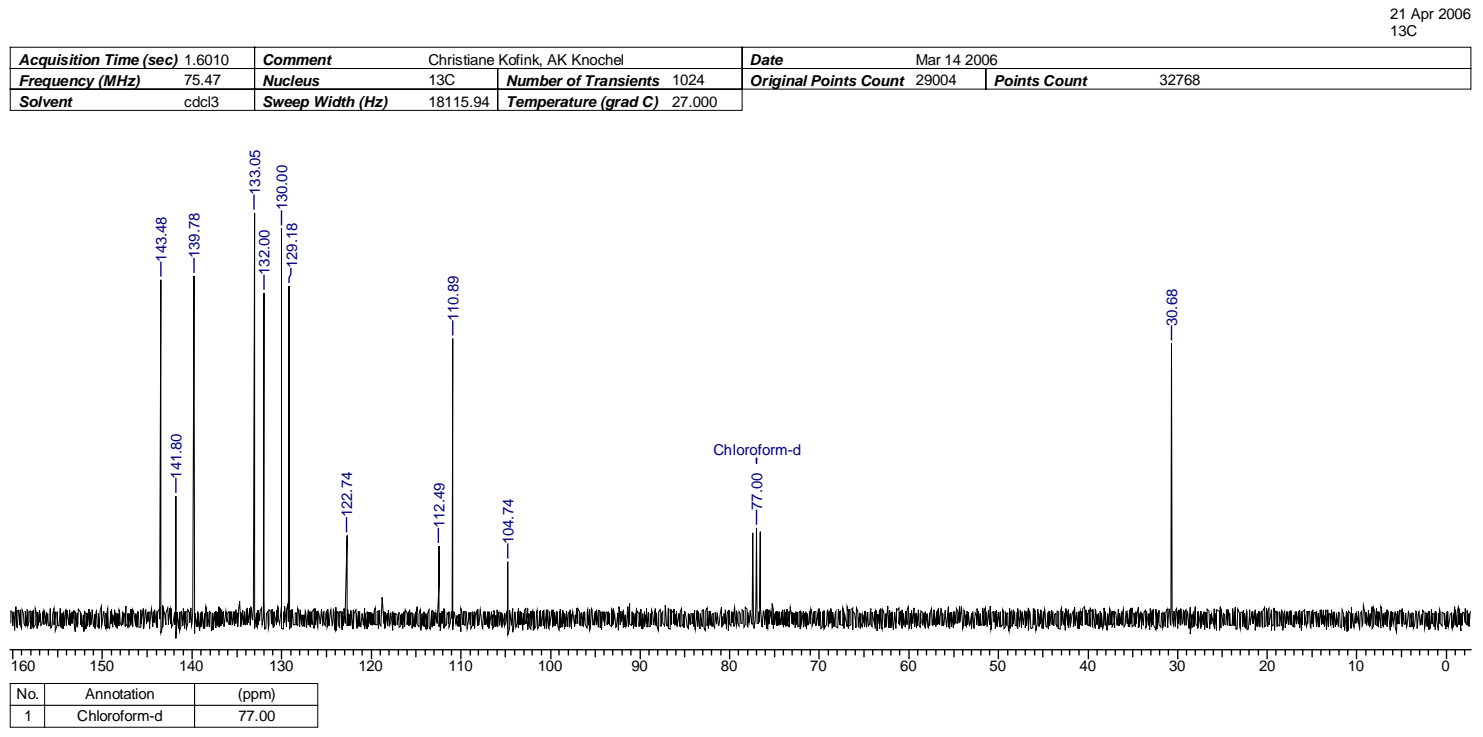




\section{Ethyl 4-(furan-3-ylmethyl)benzoate $(2 \mathbf{j})$}

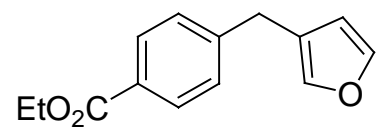

Prepared according to the TP 2. To a solution of ethyl 4-iodobenzoate $(207 \mathrm{mg}, 0.75 \mathrm{mmol})$ in $0.5 \mathrm{~mL}$ DME, $i \operatorname{PrMgCl}(0.89 \mathrm{~mL}, 0.80 \mathrm{mmol}, 0.9 \mathrm{M}$ in THF $)$ is added dropwise via syringe at $-20{ }^{\circ} \mathrm{C}$. The solution is stirred for $20 \mathrm{~min}$, than $\mathrm{CuCl}(5.0 \mathrm{mg}, 10 \mathrm{~mol} \%)$ and $\mathrm{P}(\mathrm{OEt})_{3}(17 \mathrm{mg}$, $20 \mathrm{~mol} \%$ ) is added. This mixture is added over $30 \mathrm{~min}$ via canula to a prewarmed mixture of diethyl furan-3-ylmethyl phosphate $(117 \mathrm{mg}, 0.50 \mathrm{mmol})$ and TBAI (19 mg, $10 \mathrm{~mol} \%)$. The reaction mixture is heated at $60{ }^{\circ} \mathrm{C}$ for $1 \mathrm{~h}$. The usual workup and purification by flash chromatography (pentane/ether 19:1) yielded $\mathbf{2} \mathbf{j}$ as colourless liquid (82 $\mathrm{mg}, \mathbf{7 1 \%}$ ).

${ }^{1} \mathbf{H}-\mathbf{N M R}\left(\mathrm{CDCl}_{3}, 300 \mathrm{MHz}, 25^{\circ} \mathrm{C}\right): \delta=8.00(\mathrm{~d}, J=8.3 \mathrm{~Hz}, 2 \mathrm{H}), 7.39$ (s br, $\left.1 \mathrm{H}\right), 7.30(\mathrm{~d}, J=$ $8.3 \mathrm{~Hz}, 2 \mathrm{H}), 7.24$ (s br, 1H), 6.24 (s br, 1H), 4.39 (q, J= $7.2 \mathrm{~Hz}, 2 \mathrm{H}), 3.84$ (s, 2H), 1.41 (t, $J=$ $7.2 \mathrm{~Hz}, 3 \mathrm{H})$.

${ }^{13} \mathrm{C}-\mathrm{NMR}\left(\mathrm{CDCl}_{3}, 75 \mathrm{MHz}, 25{ }^{\circ} \mathrm{C}\right): \delta=166.5,145.6,143.2,139.7,129.8,128.6,128.5$, 123.4, 111.1, 60.8, 31.2, 14.3.

IR (KBr): 2982 (w), 1710 (vs), 1611 (m), 1416 (w), 1367 (w), 1272 (vs), 1177 (m), 1101 (s), $1020(\mathrm{~s}), 873(\mathrm{~m}), 772(\mathrm{w}), 719(\mathrm{~m})$.

MS (70 eV, EI), m/z (\%): $230\left(85, \mathrm{M}^{+}\right), 185$ (100), 157 (61), 128 (33), 81 (4).

HRMS $m / z$ : calcd. for $\mathrm{C}_{14} \mathrm{H}_{14} \mathrm{O}_{3}: 230.0943$; found: 230.0939 . 
Ethyl 4-(furan-3-ylmethyl)benzoate (2j)

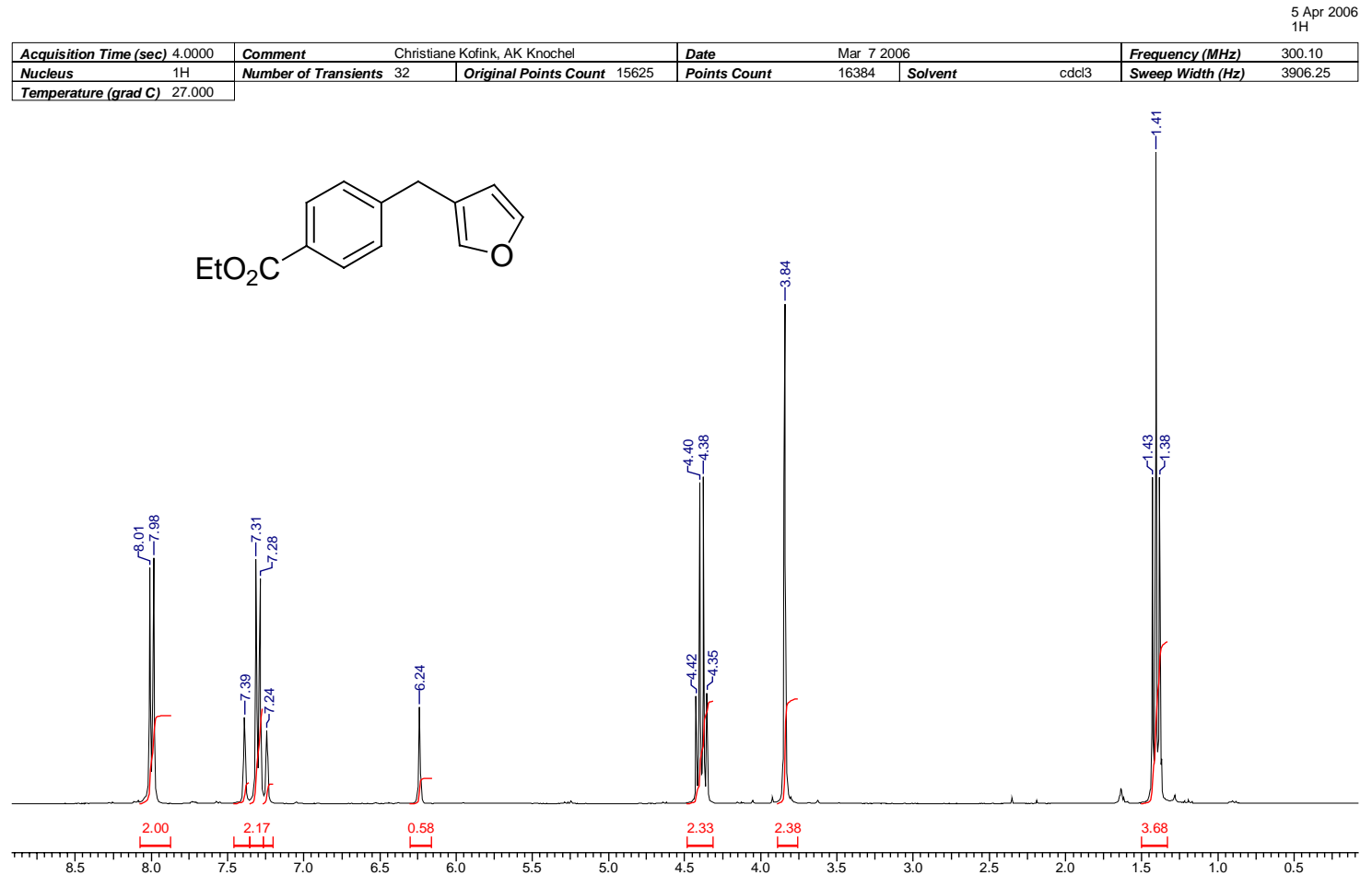

\section{Ethyl 4-(furan-3-ylmethyl)benzoate (2j)}

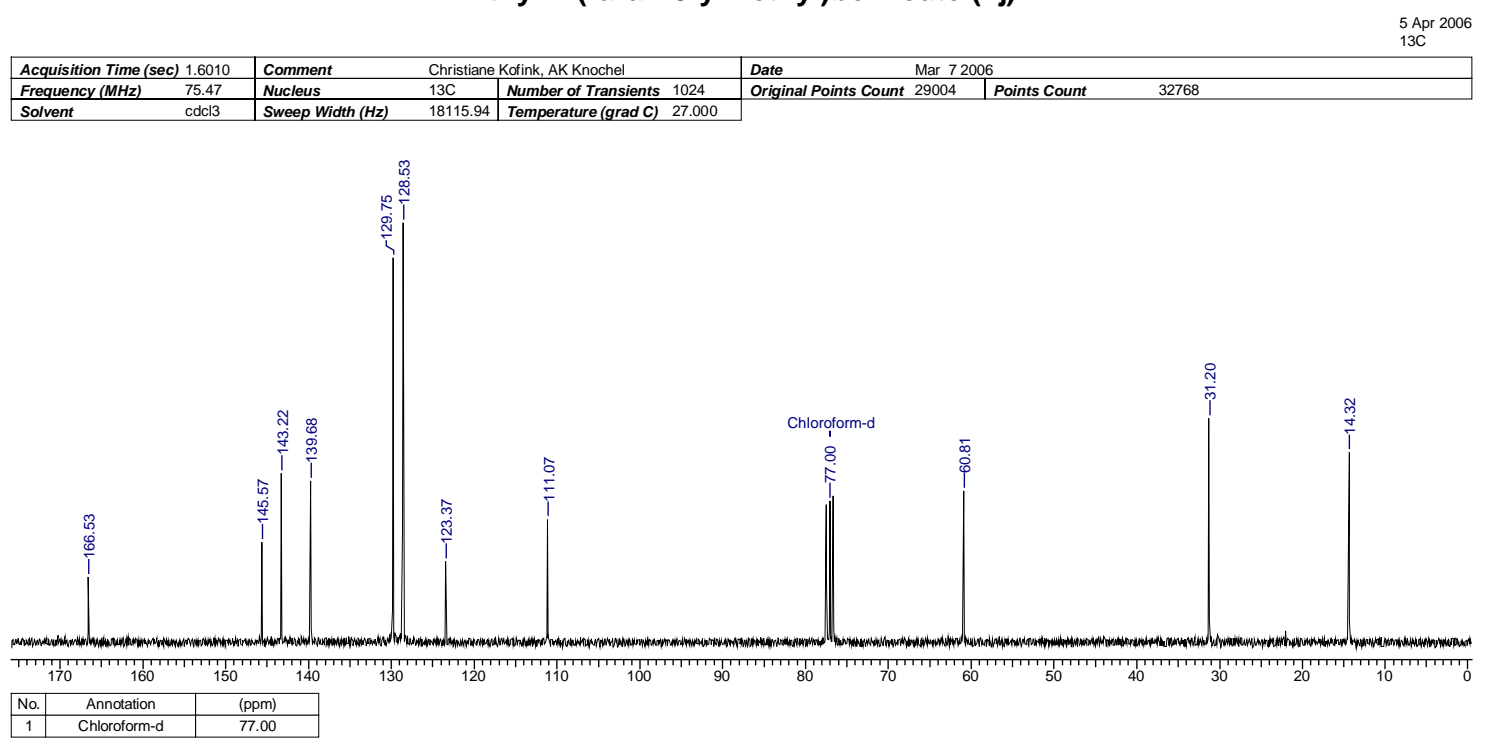




\section{Ethyl 2-(thiophen-2-ylmethyl)benzoate (2k)}

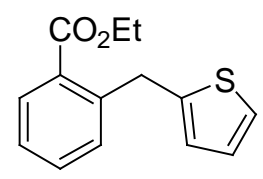

Prepared according to the TP 2. To a solution of ethyl 2-iodobenzoate $(207 \mathrm{mg}, 0.75 \mathrm{mmol})$ in $0.5 \mathrm{~mL}$ DME, $i \operatorname{PrMgCl}(0.89 \mathrm{~mL}, 0.80 \mathrm{mmol}, 0.9 \mathrm{M}$ in THF $)$ is added dropwise via syringe at $-20^{\circ} \mathrm{C}$. The solution is stirred for $3 \mathrm{~h}$, than $\mathrm{CuCl}(5.0 \mathrm{mg}, 10 \mathrm{~mol} \%)$ and $\mathrm{P}(\mathrm{OEt})_{3}(17 \mathrm{mg}$, $20 \mathrm{~mol} \%$ ) is added. This mixture is added over $30 \mathrm{~min}$ via canula to a prewarmed mixture of diethyl thiophen-2-ylmethyl phosphate $(125 \mathrm{mg}, 0.50 \mathrm{mmol})$ and TBAI (19 mg, $10 \mathrm{~mol} \%)$. The reaction mixture is heated at $60{ }^{\circ} \mathrm{C}$ for $1 \mathrm{~h}$. The usual workup and purification by flash chromatography (pentane/ether 19:1) yielded $\mathbf{2 k}$ as colourless liquid (75 mg, 61\%).

${ }^{1}$ H-NMR $\left(\mathrm{CDCl}_{3}, 300 \mathrm{MHz}, 25^{\circ} \mathrm{C}\right): \delta=7.95(\mathrm{~d}, J=7.6 \mathrm{~Hz}, 1 \mathrm{H}), 7.76(\mathrm{~m}, 1 \mathrm{H}), 7.32(\mathrm{~d}, J=$ $7.6 \mathrm{~Hz}, 2 \mathrm{H}), 7.14$ (d, J=5.1 Hz, 1H), 6.91 (s br, 1H), 6.79 (s br, 1H), 4.59 (s, 2H), 4.36 (q, J= $7.1 \mathrm{~Hz}, 2 \mathrm{H}), 1.37$ (t, $J=7.1 \mathrm{~Hz}, 3 \mathrm{H})$.

${ }^{13} \mathrm{C}-\mathrm{NMR}\left(\mathrm{CDCl}_{3}, 75 \mathrm{MHz}, 25{ }^{\circ} \mathrm{C}\right): \delta=167.4,143.8,141.6,132.0,131.0,130.7,129.7$, $126.6,125.1,123.7,60.9,34.0,29.6,14.2$.

IR (KBr): 3070 (w), 2980 (w), 1712 (vs), 1601 (w), 1577 (w), 1440 (w), 1366 (w), 1292 (m), 1259 (s), 1171 (w), 1109 (m), 1073 (s), 1047 (w), 850 (w), 740 (w), 692 (m).

MS (70 eV, EI), m/z (\%): 246 (5, M+ $), 200$ (100), 171 (23), 133 (28), 128 (4), 77 (2).

HRMS $m / z$ : calcd. for $\mathrm{C}_{14} \mathrm{H}_{14} \mathrm{O}_{2} \mathrm{~S}: 246.0715$; found: 246.0711 . 
Ethyl 2-(thiophen-2-ylmethyl)benzoate (2k)

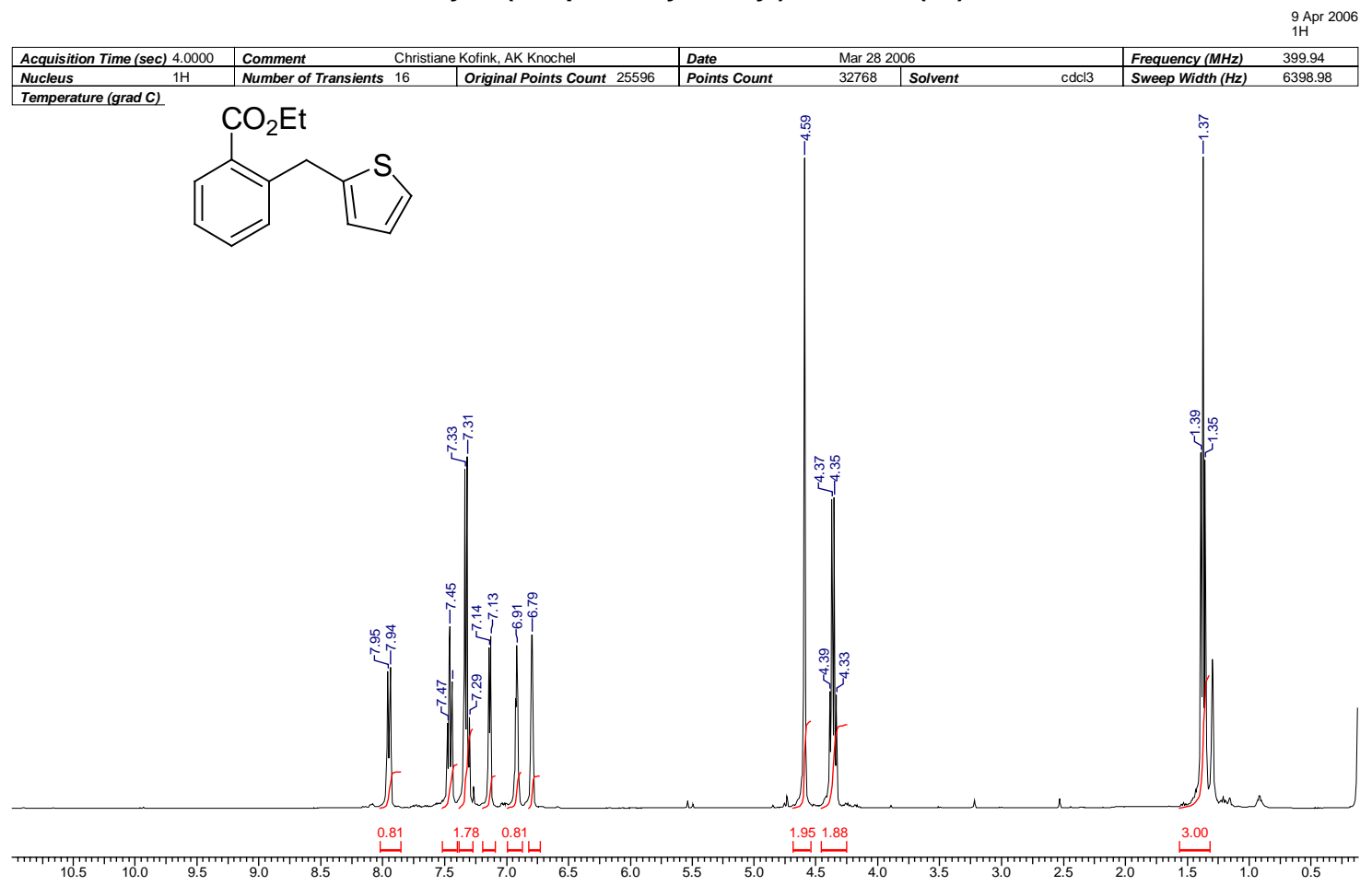

\section{Ethyl 2-(thiophen-2-ylmethyl)benzoate (2k)}

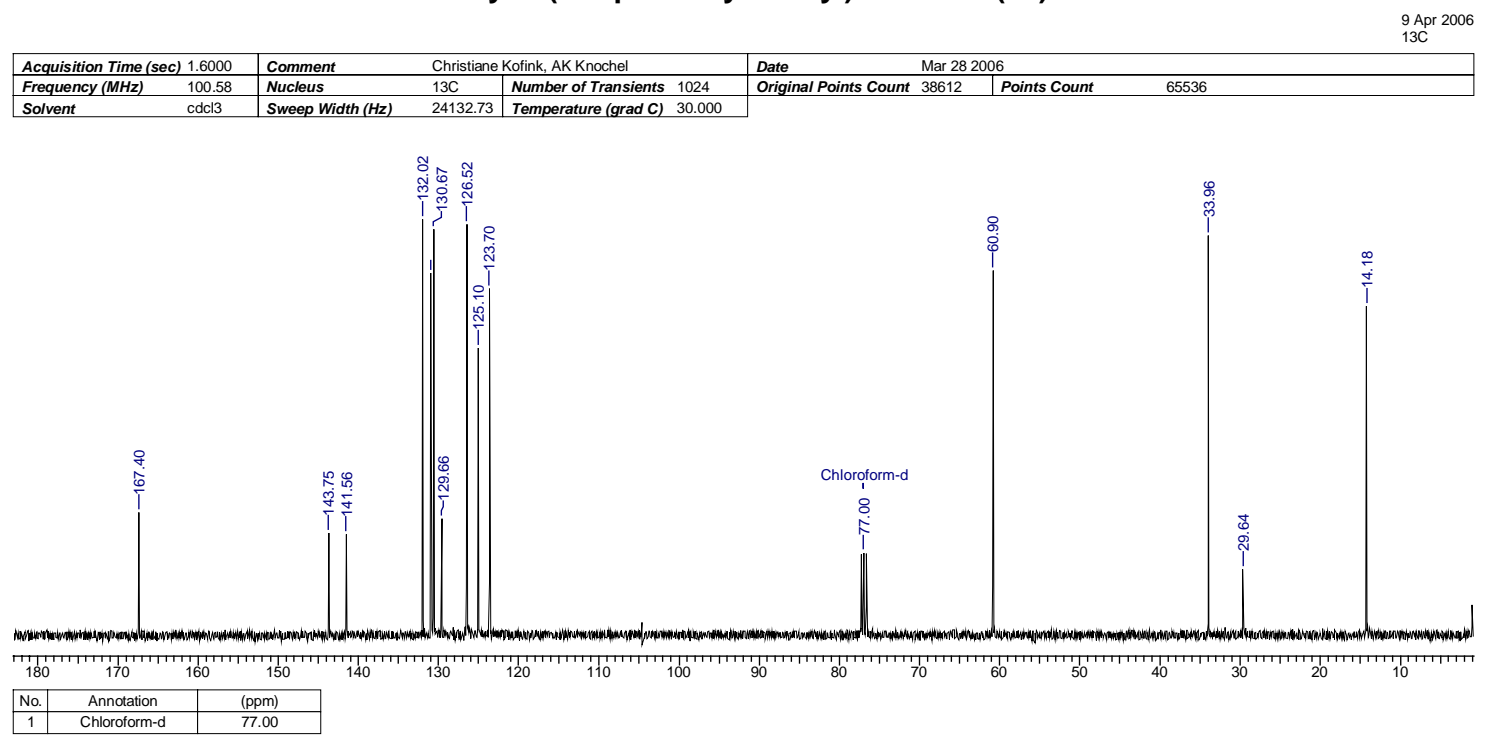




\section{Ethyl 2-(thiophen-3-ylmethyl)benzoate (2l)}

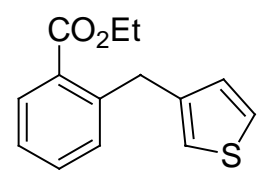

Prepared according to the TP 2. To a solution of ethyl 2-iodobenzoate $(207 \mathrm{mg}, 0.75 \mathrm{mmol})$ in $0.5 \mathrm{~mL}$ DME, $i \operatorname{PrMgCl}(0.89 \mathrm{~mL}, 0.80 \mathrm{mmol}, 0.9 \mathrm{M}$ in THF $)$ is added dropwise via syringe at $-20^{\circ} \mathrm{C}$. The solution is stirred for $3 \mathrm{~h}$, than $\mathrm{CuCl}(5.0 \mathrm{mg}, 10 \mathrm{~mol} \%)$ and $\mathrm{P}(\mathrm{OEt})_{3}(17 \mathrm{mg}$, $20 \mathrm{~mol} \%$ ) is added. This mixture is added over $30 \mathrm{~min}$ via canula to a prewarmed mixture of diethyl thiophen-3-ylmethyl phosphate $(125 \mathrm{mg}, 0.50 \mathrm{mmol})$ and TBAI (19 mg, $10 \mathrm{~mol} \%)$. The reaction mixture is heated at $60{ }^{\circ} \mathrm{C}$ for $1 \mathrm{~h}$. The usual workup and purification by flash chromatography (pentane/ether 19:1) yielded $\mathbf{2 l}$ as colourless liquid (101 $\mathrm{mg}, 82 \%)$.

${ }^{1} \mathbf{H}-\mathbf{N M R}\left(\mathrm{CDCl}_{3}, 300 \mathrm{MHz}, 25{ }^{\circ} \mathrm{C}\right): \delta=7.93(\mathrm{~d}, J=7.8 \mathrm{~Hz}, 1 \mathrm{H}), 7.45(\mathrm{t}, J=7.4 \mathrm{~Hz}, 1 \mathrm{H}), 7.28$ (m, 3H), 6.95 (d, J=4.9 Hz, 1H), 6.89 (s br, 1H), 4.40 (s, 2H), 4.33 (q, J=7.1 Hz, 2H), 1.34 (t, $J=7.1 \mathrm{~Hz}, 3 \mathrm{H})$.

${ }^{13} \mathrm{C}-\mathrm{NMR}\left(\mathrm{CDCl}_{3}, 75 \mathrm{MHz}, 25{ }^{\circ} \mathrm{C}\right): \delta=167.5,141.7,141.2,131.8,131.0,130.5,130.0$, $128.4,126.2,125.1,121.1,60.8,34.5,14.1$.

IR (KBr): 2980 (w), 1712 (vs), 1601 (w), 1576 (w), 1487 (w), 1445 (w), 1365 (w), 1255 (s), 1126 (m), 1073 (s), 1126 (m), 1073 (s), 1047 (w), 940 (w), 756 (m), 714 (m).

MS (70 eV, EI), m/z (\%): 246 (23, M+), 200 (100), 171 (46), 133 (52), 105 (4), 77 (3).

HRMS $m / z$ : calcd. for $\mathrm{C}_{14} \mathrm{H}_{14} \mathrm{O}_{2} \mathrm{~S}: 246.0715$; found: 246.0721 . 
Ethyl 2-(thiophen-3-ylmethyl)benzoate (2I)

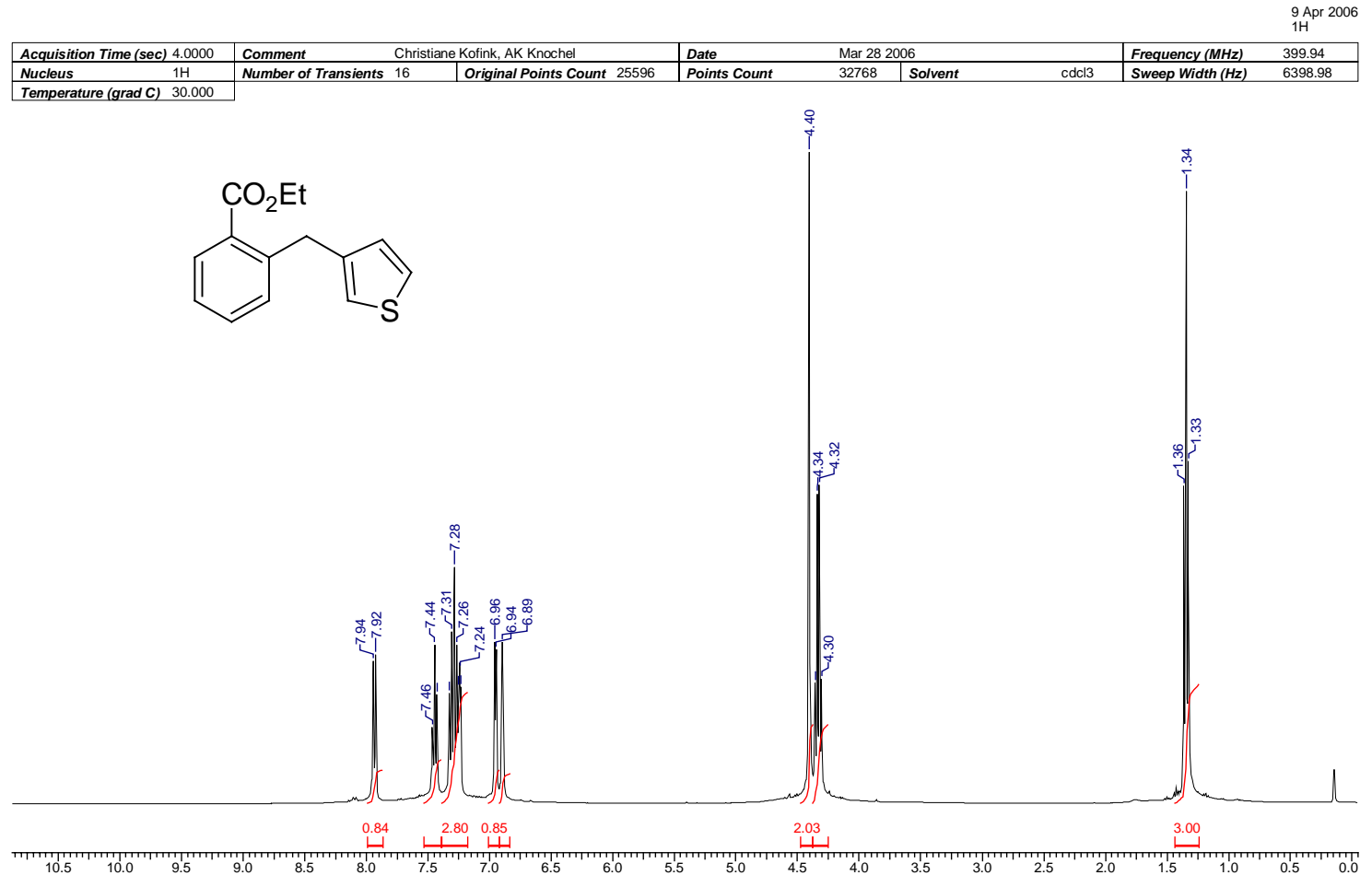

\section{Ethyl 2-(thiophen-3-ylmethyl)benzoate (2l)}
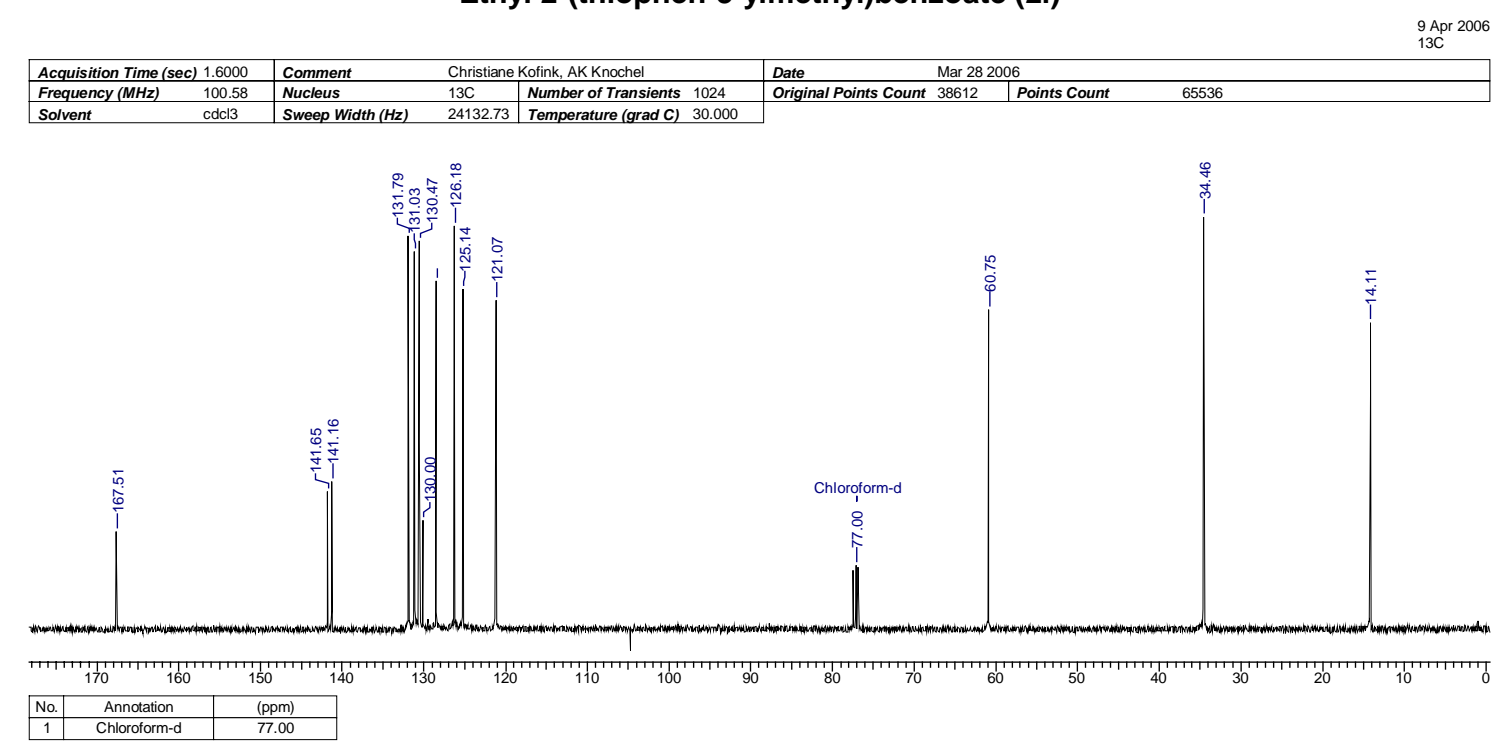


\section{Methyl 4-((2-methyl-1-tosyl-1H-indol-3-yl)methyl)benzoate (2m)}

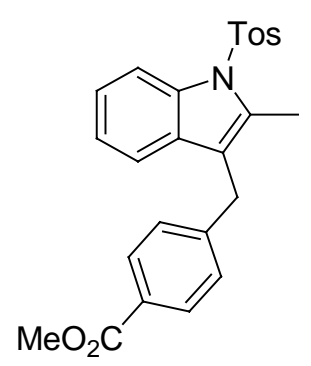

Prepared according to the TP 2. To a solution of 3-iodo-2-methyl-1-tosyl-1H-indole (308 mg, $0.75 \mathrm{mmol})$ in $0.5 \mathrm{~mL} \mathrm{DME}, i \mathrm{PrMgCl}(0.89 \mathrm{~mL}, 0.80 \mathrm{mmol}, 0.9 \mathrm{M}$ in THF$)$ is added dropwise via syringe at $-20{ }^{\circ} \mathrm{C}$. The solution is stirred for $3 \mathrm{~h}$, than $\mathrm{CuCl}(5.0 \mathrm{mg}, 10 \mathrm{~mol} \%)$ and $\mathrm{P}(\mathrm{OEt})_{3}(17 \mathrm{mg}, 20 \mathrm{~mol} \%)$ is added. This mixture is added over $30 \mathrm{~min}$ via canula to a prewarmed mixture of methyl 4-((diethoxyphosphoryloxy)methyl)benzoate (151 mg, $0.50 \mathrm{mmol})$ and TBAI (19 mg, $10 \mathrm{~mol} \%)$. The reaction mixture is heated at $60{ }^{\circ} \mathrm{C}$ for $1 \mathrm{~h}$. The usual workup and purification by flash chromatography (pentane/ether 9:1) yielded $\mathbf{2 m}$ as colourless solid (151 mg, 70\%).

Mp.: $121^{\circ} \mathrm{C}$.

${ }^{1}$ H-NMR $\left(\mathrm{CDCl}_{3}, 300 \mathrm{MHz}, 25{ }^{\circ} \mathrm{C}\right): \delta=8.25(\mathrm{~d}, J=8.5 \mathrm{~Hz}, 1 \mathrm{H}), 7.89(\mathrm{~d}, J=8.2 \mathrm{~Hz}, 2 \mathrm{H})$, $7.65(\mathrm{~d}, J=8.2 \mathrm{~Hz}, 2 \mathrm{H}), 7.32-7.09$ (m, 7H), 4.03 (s, 2H), 3.90 (s, 3H), 2.59 (s, 3H), 2.38 (s, $3 \mathrm{H})$.

${ }^{13} \mathrm{C}-\mathrm{NMR}\left(\mathrm{CDCl}_{3}, 75 \mathrm{MHz}, 25{ }^{\circ} \mathrm{C}\right): \delta=166.8,144.9,144.7,136.6,136.1,133.9,130.3$, 129.8, 129.7, 128.1, 127.9, 126.2, 124.1, 123.5, 188.5, 118.34, 114.8, 51.9, 29.9, 21.5, 12.9 .

IR (KBr): 1716 (s), 1610 (m), 1453 (m), 1361 (s), 1277 (vs), 1236 (m), 1171 (vs), 1106 (s), 1089 (s), 988 (m), 938 (m), 877 (w), 811 (m), 744 (vs), 704 (m), 686 (m).

MS (70 eV, EI), m/z (\%): $433\left(41, \mathrm{M}^{+}\right), 278$ (100), 218 (10), 178 (6), 91 (8).

HRMS $m / z$ : calcd. for $\mathrm{C}_{25} \mathrm{H}_{23} \mathrm{NO}_{4} \mathrm{~S}: 433.1348$; found: 433.1327 . 


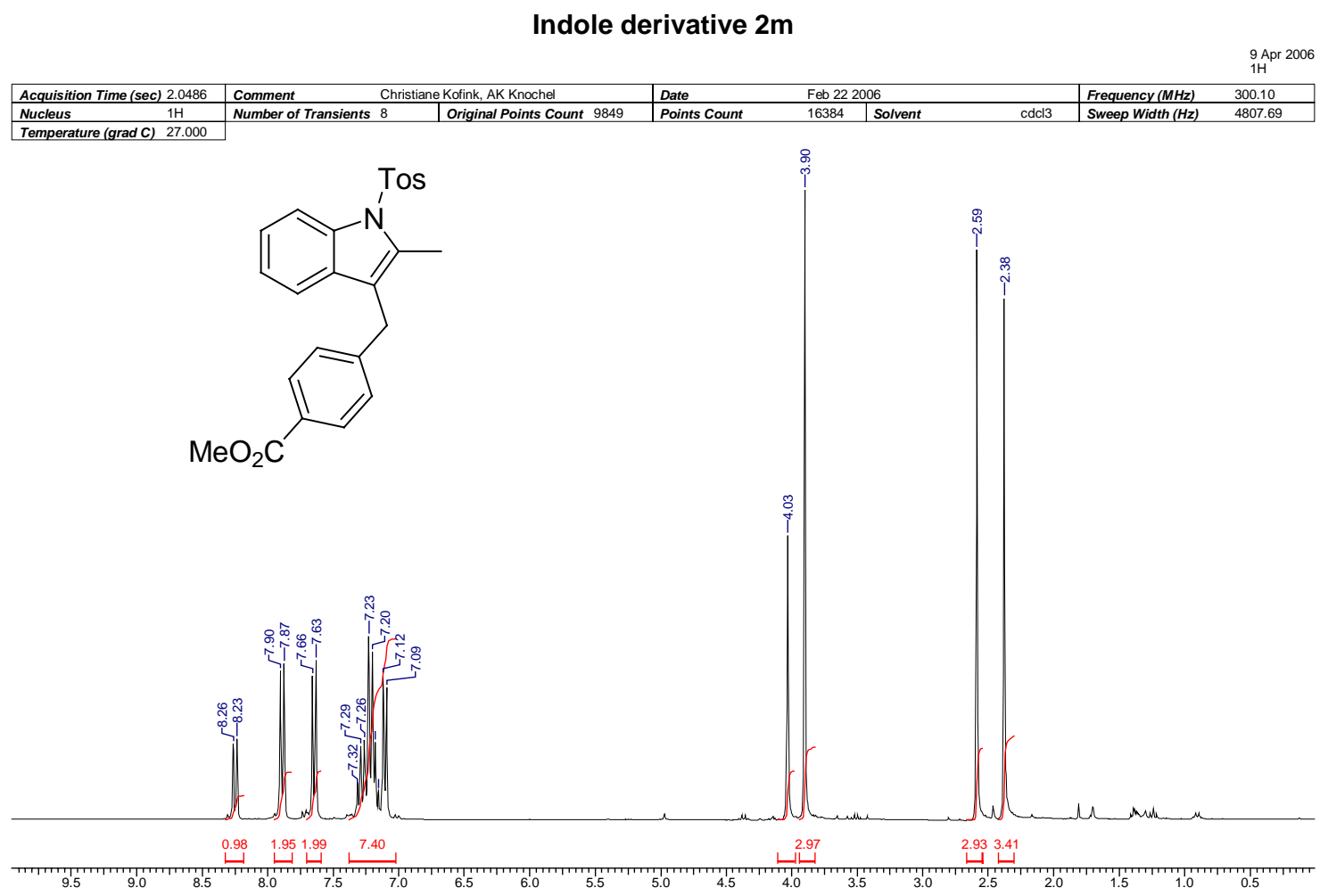

\section{Indole derivative $\mathbf{2 m}$}

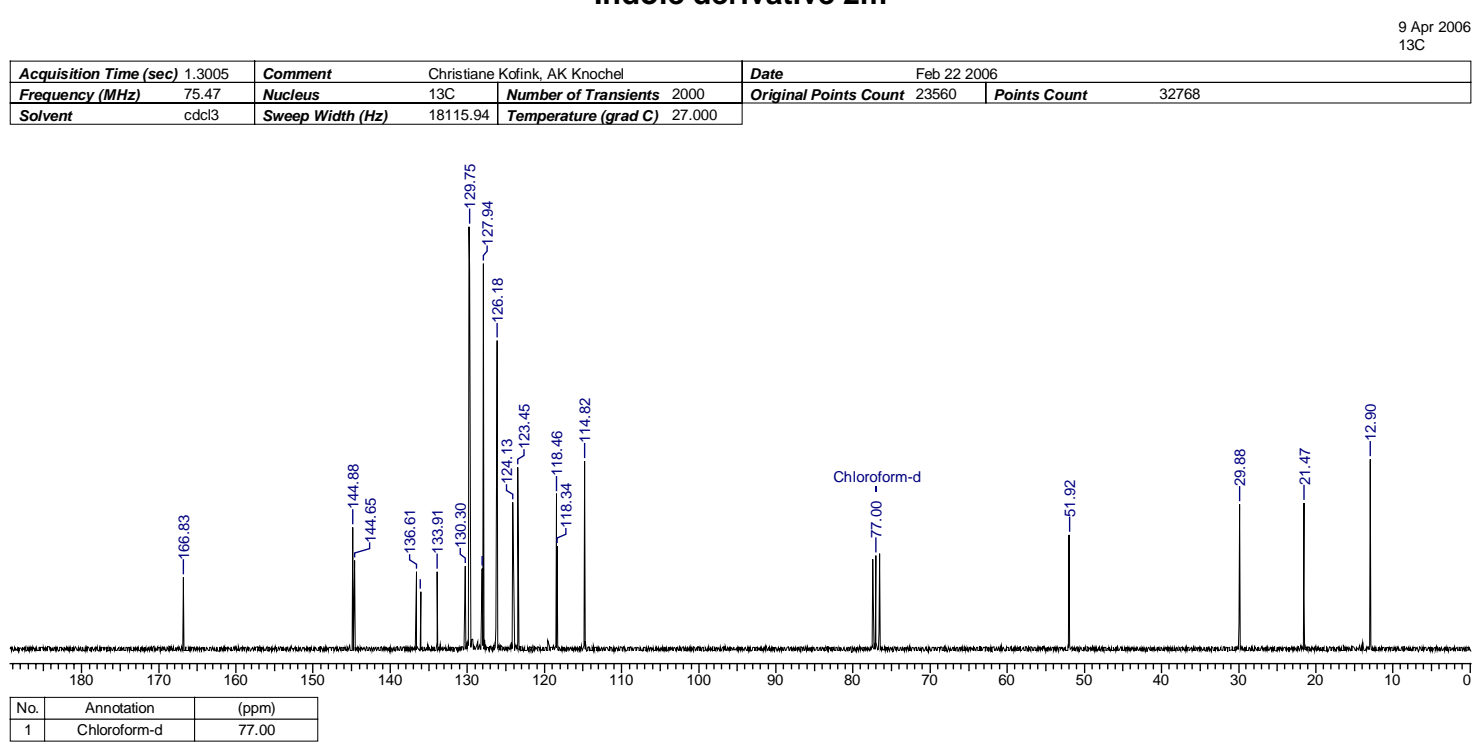




\section{Methyl 4-((1-benzyl-2-methyl-1H-indol-3-yl)methyl)benzoate (2n)}

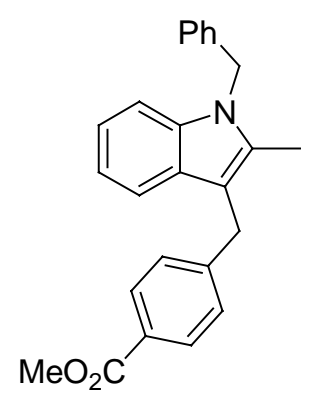

Prepared according to the TP 2. To a solution of 1-benzyl-3-iodo-2-methyl-1H-indole (260 $\mathrm{mg}, 0.75 \mathrm{mmol})$ in $0.5 \mathrm{~mL} \mathrm{DME}, i \mathrm{PrMgCl}(0.89 \mathrm{~mL}, 0.80 \mathrm{mmol}, 0.9 \mathrm{M}$ in THF) is added dropwise via syringe at $-20{ }^{\circ} \mathrm{C}$. The solution is stirred for $3 \mathrm{~h}$, than $\mathrm{CuCl}(5.0 \mathrm{mg}, 10 \mathrm{~mol} \%)$ and $\mathrm{P}(\mathrm{OEt})_{3}(17 \mathrm{mg}, 20 \mathrm{~mol} \%)$ is added. This mixture is added over $30 \mathrm{~min}$ via canula to a prewarmed mixture of methyl 4-((diethoxyphosphoryloxy)methyl)benzoate (151 mg, $0.50 \mathrm{mmol})$ and TBAI (19 mg, $10 \mathrm{~mol} \%)$. The reaction mixture is heated at $60{ }^{\circ} \mathrm{C}$ for $1 \mathrm{~h}$. The usual workup and purification by flash chromatography (pentane/ether 9:1) yielded 2n as colourless solid (143 mg, 77\%).

Mp.: $115^{\circ} \mathrm{C}$.

${ }^{1} \mathbf{H}$-NMR $\left(\mathrm{CDCl}_{3}, 300 \mathrm{MHz}, 25{ }^{\circ} \mathrm{C}\right): \delta=7.98(\mathrm{~d}, J=7.9 \mathrm{~Hz}, 2 \mathrm{H}), 7.48(\mathrm{~d}, J=7.3 \mathrm{~Hz}, 1 \mathrm{H})$, 7.36-7.27 (m, 6H), 7.20-7.07 (m, 2H), 7.04-7.01 (m, 2H), 5.38 (s, 2H), 4.23 (s, 2H), 3.93 (s, $3 \mathrm{H}), 2.35(\mathrm{~s}, 3 \mathrm{H})$.

${ }^{13} \mathrm{C}-\mathrm{NMR}\left(\mathrm{CDCl}_{3}, 75 \mathrm{MHz}, 25{ }^{\circ} \mathrm{C}\right): \delta=167.1,147.3,137.9,136.6,133.6,129.6,128.7$, 128.2, 128.0, 127.7, 127.2, 125.9, 121.0, 119.3, 118.2, 109.6, 109.0, 51.9, 46.6, 30.5, 10.4.

IR (KBr): 3030 (w), 2919 (w), 1721 (s), 1608 (m), 1468 (m), 1430 (m), 1279 (vs), 1194 (m), 1172 (s), 1101 (s), 1020 (m), 923 (w), 8118 (w), 755 (m), 737 (vs), 715 (s), 696 (s).

MS (70 eV, EI), m/z (\%): 369 (100, M M $^{+}, 354$ (14), 338 (4), 278 (10), 234 (22), 218 (7), 91 (77), 65 (4).

HRMS $m / z$ : calcd. for $\mathrm{C}_{25} \mathrm{H}_{23} \mathrm{NO}_{2}$ : 369.1729; found: 369.1737 . 

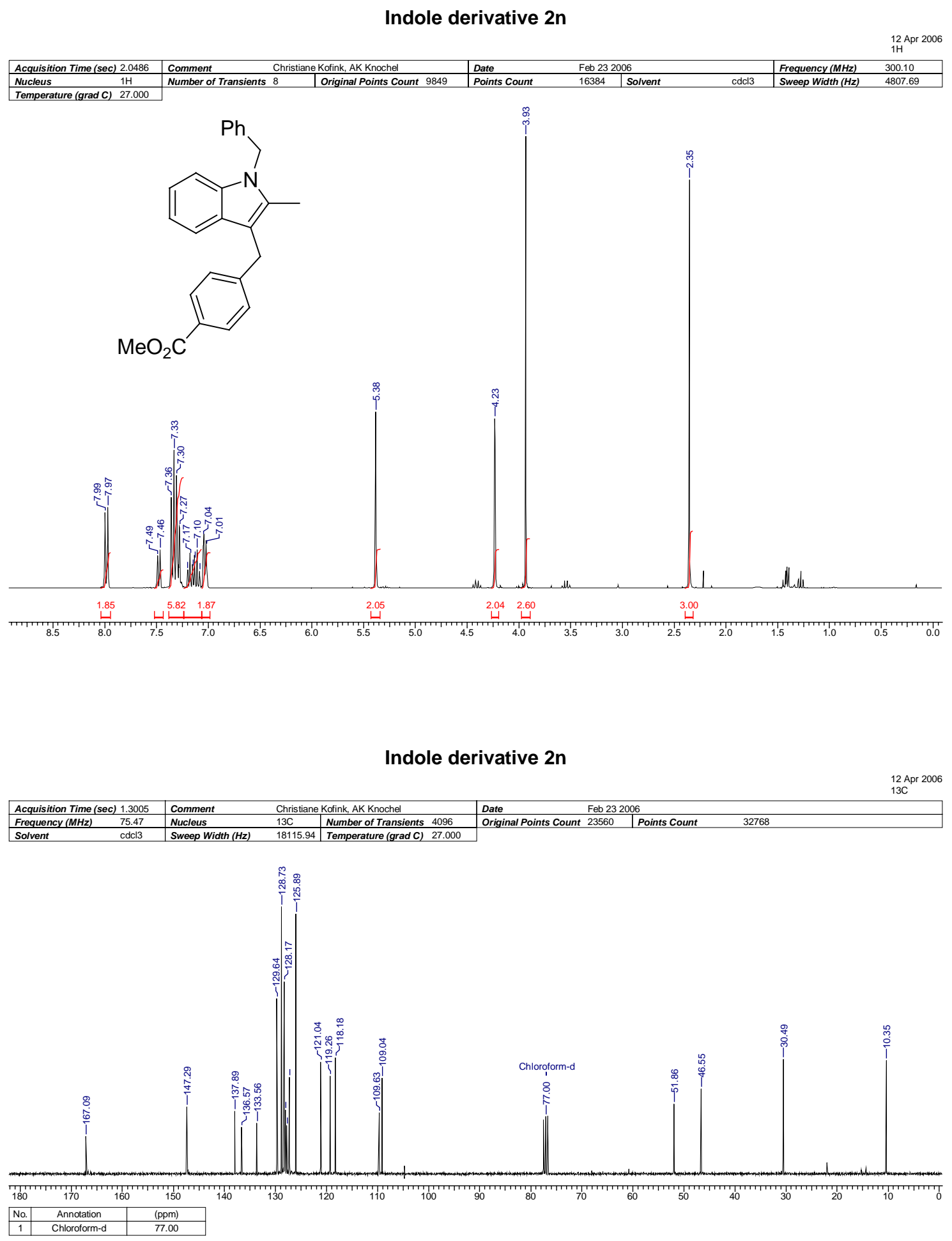


\section{1-Benzyl-3-(2-bromobenzyl)-2-methyl-1H-indole (2o)}

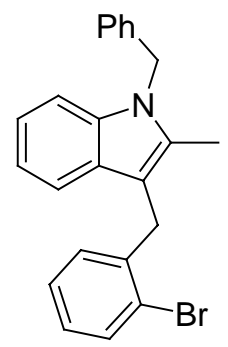

Prepared according to the TP 2. To a solution of 1-benzyl-3-iodo-2-methyl-1H-indole (260 mg, $0.75 \mathrm{mmol})$ in $0.5 \mathrm{~mL} \mathrm{DME}, i \mathrm{PrMgCl}(0.89 \mathrm{~mL}, 0.80 \mathrm{mmol}, 0.9 \mathrm{M}$ in THF) is added dropwise via syringe at $-20{ }^{\circ} \mathrm{C}$. The solution is stirred for $3 \mathrm{~h}$, than $\mathrm{CuCl}(5.0 \mathrm{mg}, 10 \mathrm{~mol} \%)$ and $\mathrm{P}(\mathrm{OEt})_{3}(17 \mathrm{mg}, 20 \mathrm{~mol} \%)$ is added. This mixture is added over $30 \mathrm{~min}$ via canula to a prewarmed mixture of methyl 2-bromobenzyl diethyl phosphate $(162 \mathrm{mg}, 0.50 \mathrm{mmol})$ and TBAI (19 mg, $10 \mathrm{~mol} \%$ ). The reaction mixture is heated at $60{ }^{\circ} \mathrm{C}$ for $1 \mathrm{~h}$. The usual workup and purification by flash chromatography (pentane/ether 19:1) yielded 20 as colourless solid (163 mg, 85\%).

Mp.: $122^{\circ} \mathrm{C}$.

${ }^{1} \mathbf{H}$-NMR $\left(\mathrm{CDCl}_{3}, 300 \mathrm{MHz}, 25{ }^{\circ} \mathrm{C}\right): \delta=7.68(\mathrm{~d}, J=9.3 \mathrm{~Hz}, 1 \mathrm{H}), 7.51(\mathrm{~d}, J=9.3 \mathrm{~Hz}, 1 \mathrm{H})$, 7.39-7.05 (m, 11H), 5.43 (s, 2H), 4.31 (s, 2H), 2.37 (s, 3H).

${ }^{13} \mathrm{C}-\mathrm{NMR}\left(\mathrm{CDCl}_{3}, 75 \mathrm{MHz}, 25{ }^{\circ} \mathrm{C}\right): \delta=140.4,137.9,136.6,134.1,132.4,129.9,128.7$, 128.2, 127.4, 127.3, 127.2, 125.9, 124.5, 121.0, 119.2, 118.4, 109.0, 108.9, 46.6, 30.7, 10.4. IR (KBr): 3028 (w), 1564 (w), 1494 (m), 1468 (m), 1452 (m), 1416 (m), 1368 (m), 1263 (w), $1178(\mathrm{w}), 1019$ (m), 928 (w), 834 (w), 748 (s), 732 (vs), 722 (s), 691 (m).

MS (70 eV, EI), m/z (\%): 389 (100, M+), 376 (13), 298 (11), 234 (31), 218 (22), 178 (10), 91 (99), 65 (6).

HRMS $m / z$ : calcd. for $\mathrm{C}_{23} \mathrm{H}_{20} \mathrm{BrN}$ : 389.0779 ; found: 389.0762 . 


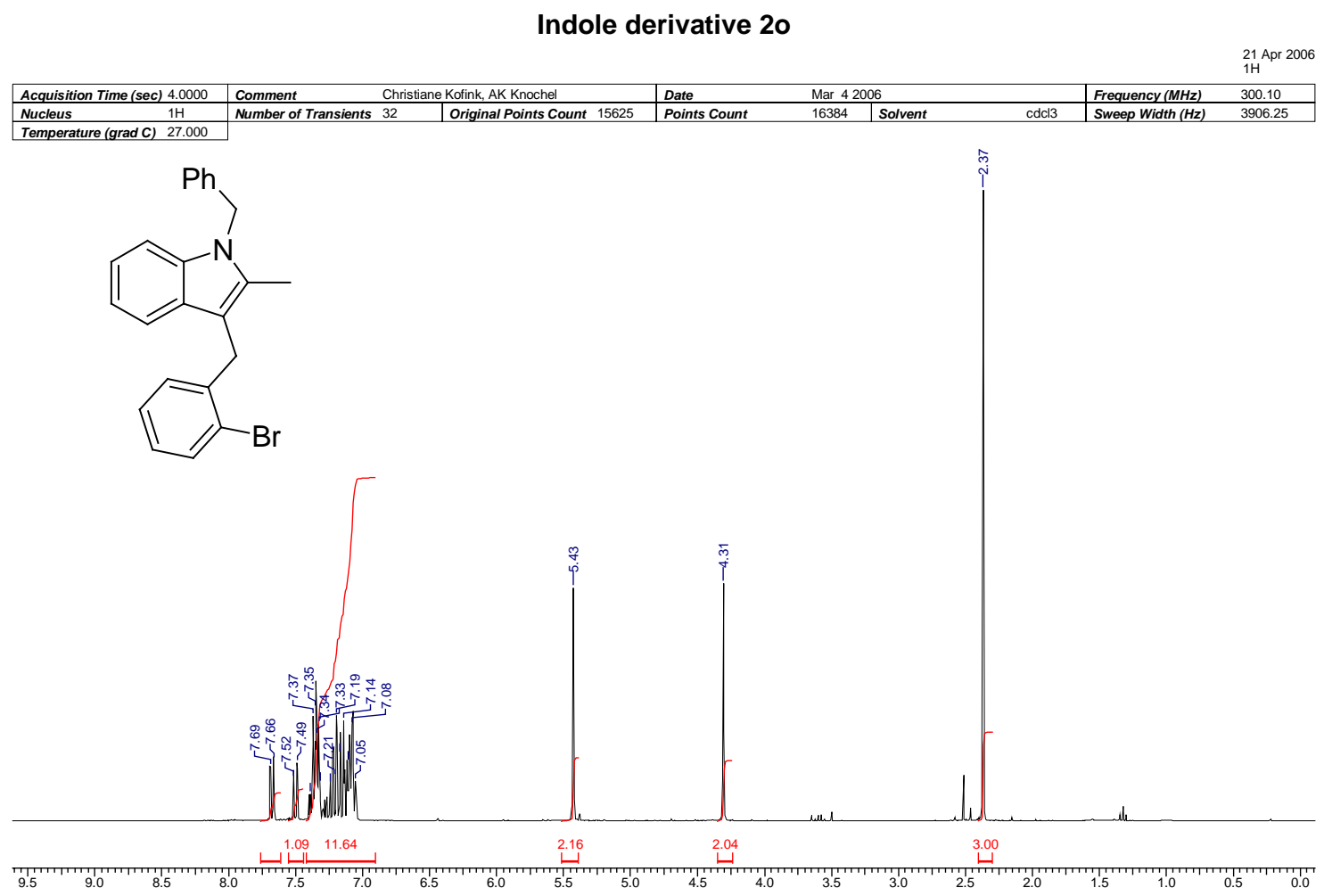

\section{Indole derivative 20}

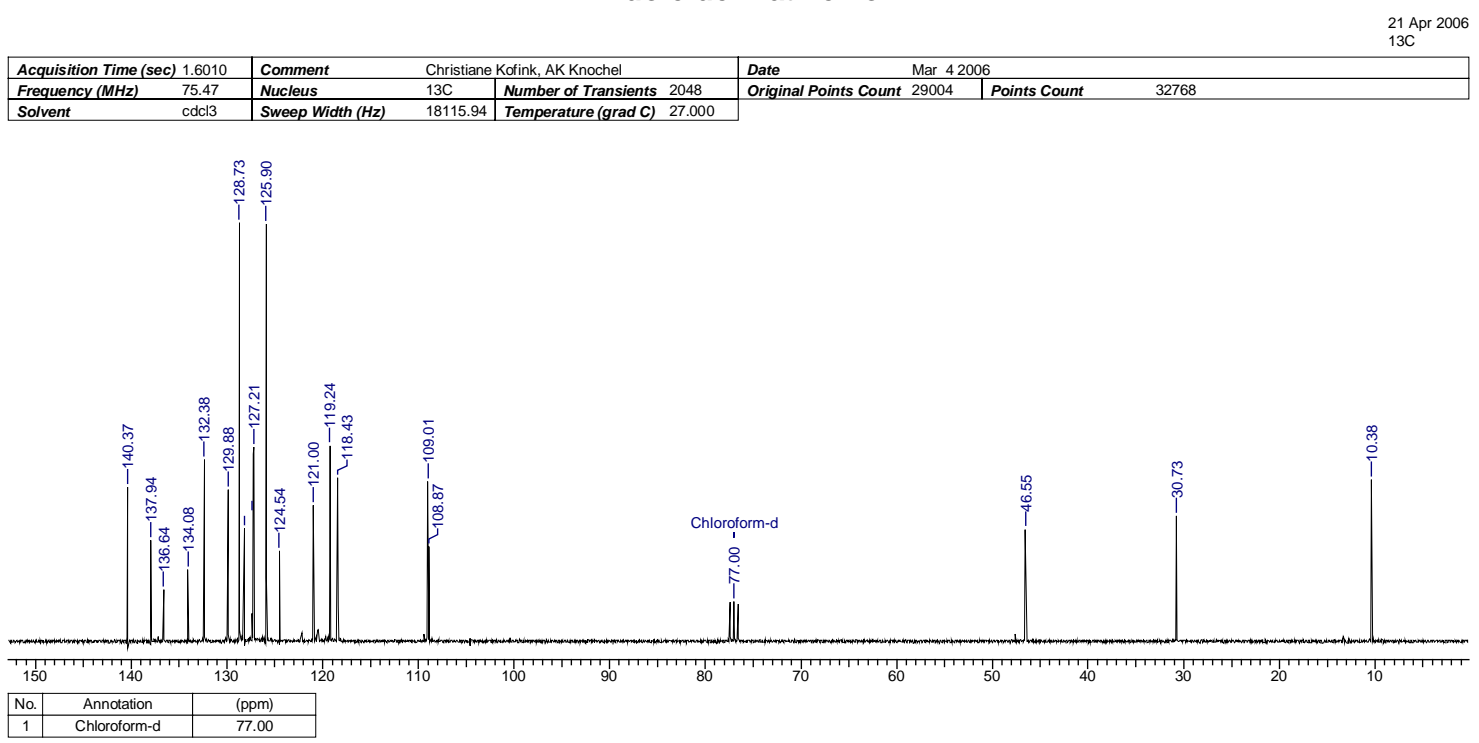




\section{2-Methyl-3-(thiophen-3-ylmethyl)-1-tosyl-1H-indole (2p)}

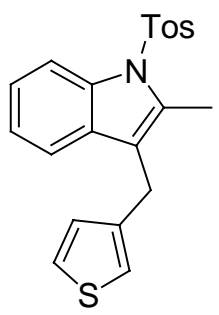

Prepared according to the TP 2. To a solution of 3-iodo-2-methyl-1-tosyl-1H-indole (308 mg, $0.75 \mathrm{mmol})$ in $0.5 \mathrm{~mL} \mathrm{DME}, i \mathrm{PrMgCl}(0.89 \mathrm{~mL}, 0.80 \mathrm{mmol}, 0.9 \mathrm{M}$ in THF $)$ is added dropwise via syringe at $-20{ }^{\circ} \mathrm{C}$. The solution is stirred for $3 \mathrm{~h}$, than $\mathrm{CuCl}(5.0 \mathrm{mg}, 10 \mathrm{~mol} \%)$ and $\mathrm{P}(\mathrm{OEt})_{3}(17 \mathrm{mg}, 20 \mathrm{~mol} \%)$ is added. This mixture is added over $30 \mathrm{~min}$ via canula to a prewarmed mixture of diethyl thiophen-3-ylmethyl phosphate $(125 \mathrm{mg}, 0.50 \mathrm{mmol})$ and TBAI (19 mg, $10 \mathrm{~mol} \%$ ). The reaction mixture is heated at $60{ }^{\circ} \mathrm{C}$ for $1 \mathrm{~h}$. The usual workup and purification by flash chromatography (pentane/ether 19:1) yielded $\mathbf{2 p}$ as colourless solid (156 mg, 82\%).

Mp:. $113{ }^{\circ} \mathrm{C}$.

${ }^{1} \mathbf{H}$-NMR $\left(\mathrm{CDCl}_{3}, 300 \mathrm{MHz}, 25{ }^{\circ} \mathrm{C}\right): \delta=8.24(\mathrm{~d}, J=8.3 \mathrm{~Hz}, 1 \mathrm{H}), 7.65(\mathrm{~d}, J=8.3 \mathrm{~Hz}, 2 \mathrm{H})$, 7.30-7.20 (m, 6H), 6.83 (d, J=4.8 Hz, 1H), 6.67 (s br, 1H), 3.96 (s, 2H), 2.58 (s, 3H), 2.37 (s, $3 \mathrm{H})$,

${ }^{13} \mathrm{C}-\mathrm{NMR}\left(\mathrm{CDCl}_{3}, 75 \mathrm{MHz}, 25{ }^{\circ} \mathrm{C}\right): \delta=144.6,139.9,136.6,136.2,133.4,130.5,129.8$, $127.8,126.2$, 125.6, 124.0, 123.4, 120.6, 119.1, 118.6, 114.8, 24.9, 21.5, 12.9.

IR (KBr): 3093 (w), 2918 (w), 1594 (w), 1452 (m), 1353 (s), 1294 (w), 1221 (m), 1168 (vs), 1150 (s), 1086 (s), 1016 (m), 974 (s), 940 (m), 867 (w), 811 (m), 767 (s), 746 (vs), 659 (s).

MS (70 eV, EI), m/z (\%): $381\left(38, \mathrm{M}^{+}\right), 226$ (100), 184 (10), 91 (6), 65 (2).

HRMS m/z: calcd. for $\mathrm{C}_{21} \mathrm{H}_{19} \mathrm{NO}_{2} \mathrm{~S}_{2}: 381.0857$; found: 381.0834 . 


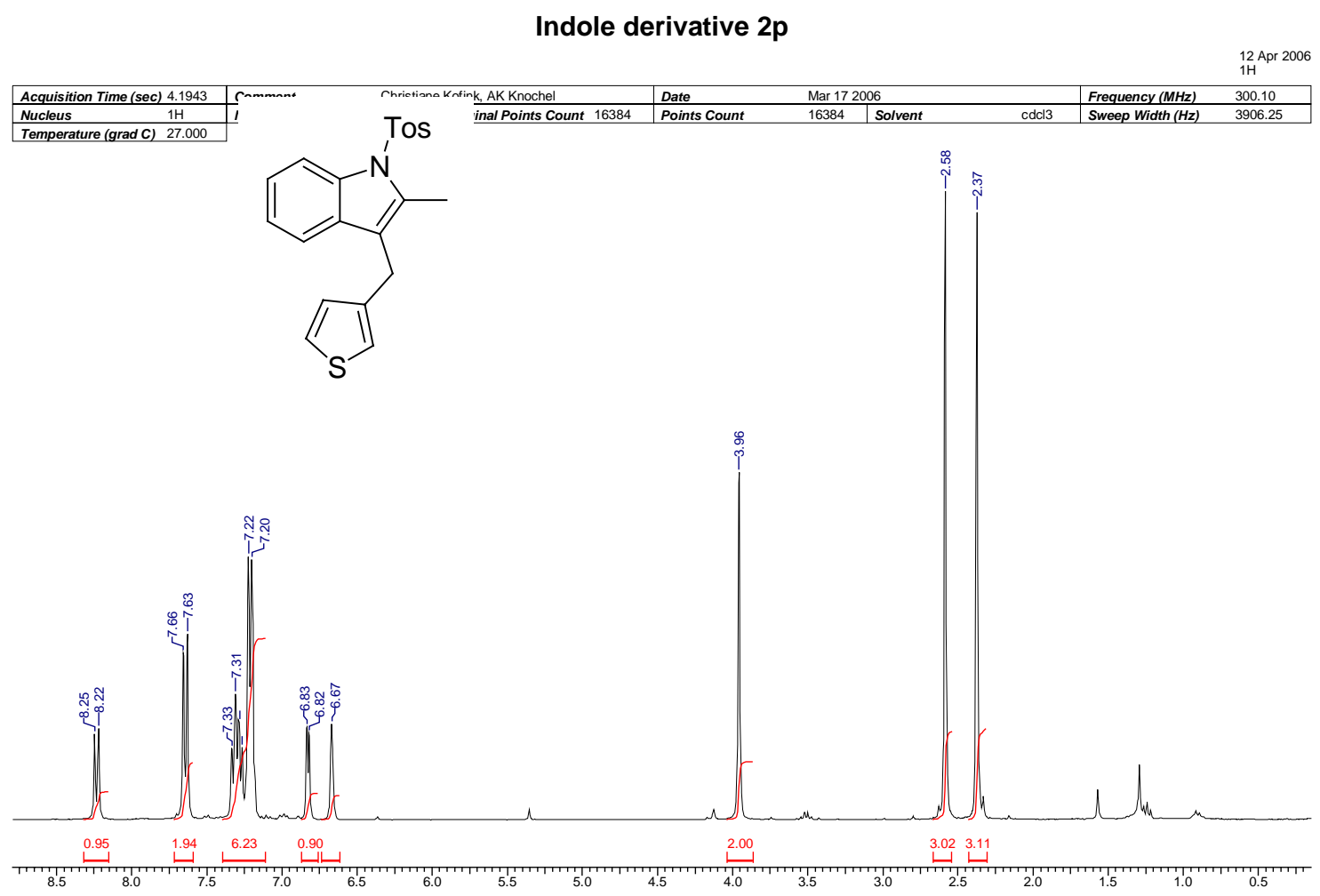

\section{Indole derivative $2 p$}

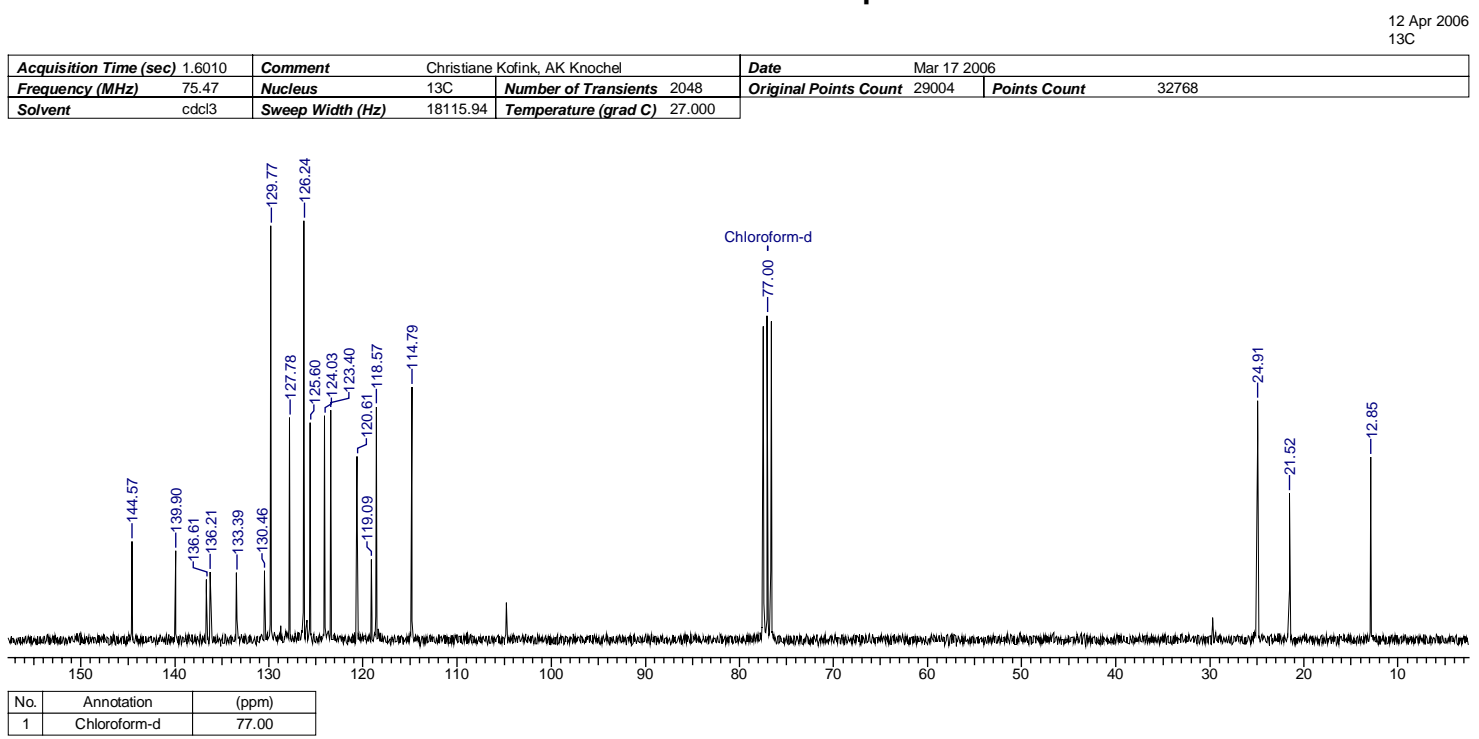




\section{1-Benzyl-2-methyl-3-(thiophen-3-ylmethyl)-1H-indole (2q)}

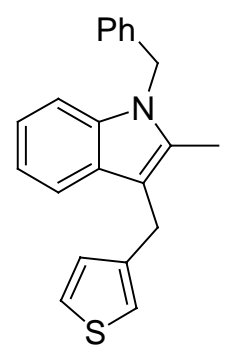

Prepared according to the TP 2. To a solution of 1-benzyl-3-iodo-2-methyl-1H-indole (260 mg, $0.75 \mathrm{mmol})$ in $0.5 \mathrm{~mL} \mathrm{DME}, i \mathrm{PrMgCl}(0.89 \mathrm{~mL}, 0.80 \mathrm{mmol}, 0.9 \mathrm{M}$ in THF) is added dropwise via syringe at $-20^{\circ} \mathrm{C}$. The solution is stirred for $3 \mathrm{~h}$, than $\mathrm{CuCl}(5.0 \mathrm{mg}, 10 \mathrm{~mol} \%)$ and $\mathrm{P}(\mathrm{OEt})_{3}(17 \mathrm{mg}, 20 \mathrm{~mol} \%)$ is added. This mixture is added over $30 \mathrm{~min}$ via canula to a prewarmed mixture of diethyl thiophen-3-ylmethyl phosphate $(125 \mathrm{mg}, 0.50 \mathrm{mmol})$ and TBAI (19 $\mathrm{mg}, 10 \mathrm{~mol} \%$ ). The reaction mixture is heated at $60{ }^{\circ} \mathrm{C}$ for $1 \mathrm{~h}$. The usual workup and purification by flash chromatography (pentane) yielded $\mathbf{2 q}$ as colourless solid (137 $\mathrm{mg}, 86 \%$ ).

Mp:. $68^{\circ} \mathrm{C}$.

${ }^{1} \mathbf{H}-\mathbf{N M R}\left(\mathrm{CDCl}_{3}, 600 \mathrm{MHz}, 25{ }^{\circ} \mathrm{C}\right): \delta=7.51-7.47(\mathrm{~m}, 1 \mathrm{H}), 7.28-7.21(\mathrm{~m}, 5 \mathrm{H}), 7.13-7.05(\mathrm{~m}$, 2H), 6.99-6.97 (m, 3H), 6.88 (s br, 1H), 5.33 (s, 2H), 4.11 (s, 2H), 2.31 (s, 3H).

${ }^{13} \mathrm{C}-\mathrm{NMR}\left(\mathrm{CDCl}_{3}, 150 \mathrm{MHz}, 25{ }^{\circ} \mathrm{C}\right): \delta=142.4,138.1,136.6,133.1,128.8,128.2,128.0$, 127.2, 126.0, 125.3, 120.9, 120.3, 119.2, 118.3, 110.4, 109.0, 104.8, 46.6, 25.3, 10.3.

IR (KBr): 3854 (w), 3745 (w), 3108 (m), 3026 (m), 2908 (m), 1653 (w), 1560 (w), 1494 (m), 1468 (vs), 1454 (s), 1418 (m), 1350 (m), 1336 (s), 1259 (w), 1178 (w), 1020 (w), 759 (m), $741(\mathrm{~s}), 713(\mathrm{~m}), 700(\mathrm{~m})$.

MS (70 eV, EI), m/z (\%): $317\left(100, \mathrm{M}^{+}\right), 302$ (33), 234 (10), 226 (16), 184 (8), 91 (82), 65 (6).

HRMS $m / z$ : calcd. for $\mathrm{C}_{21} \mathrm{H}_{19} \mathrm{NS}$ : 317.1238 ; found: 317.1214 . 


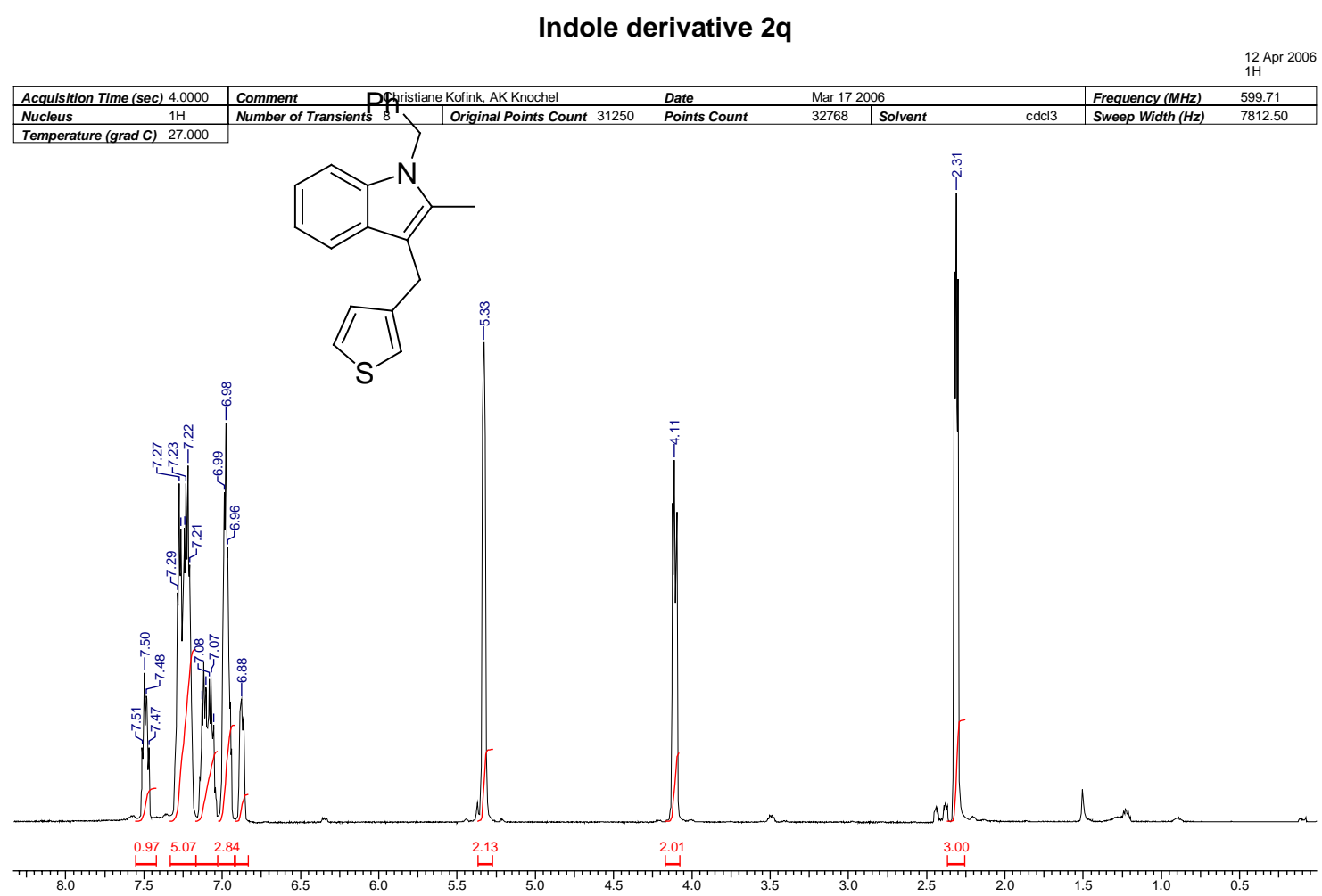

\section{Indole derivative $\mathbf{2 q}$}

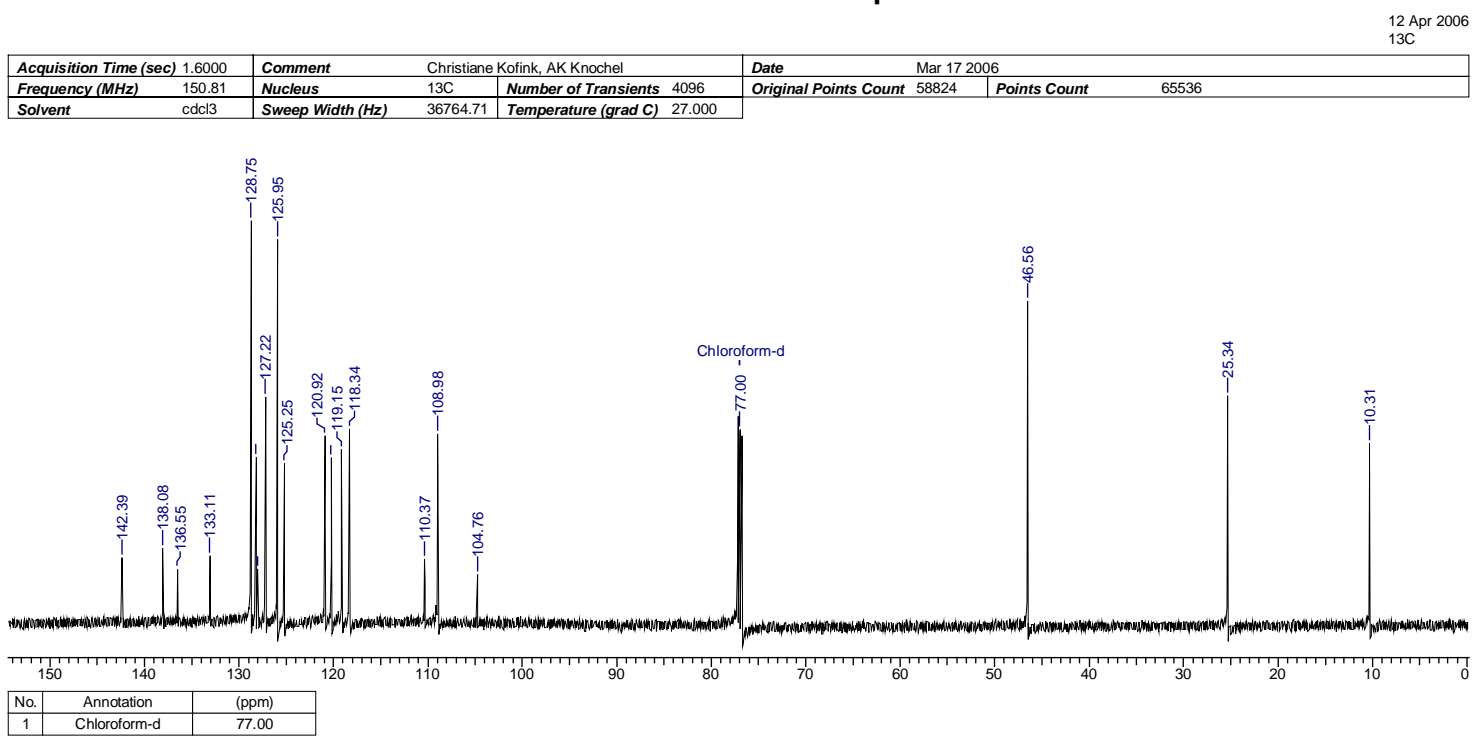




\section{Ethyl 1-benzyl-3-(furan-3-ylmethyl)-1H-indole-2-carboxylate (2r)}

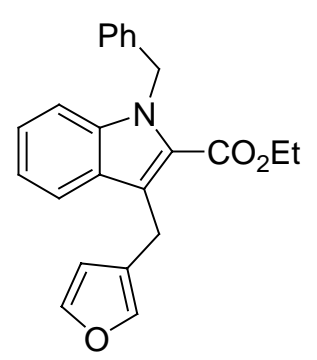

Prepared according to the TP 2. To a solution of ethyl 1-benzyl-3-iodo-1H-indole-2carboxylate $(304 \mathrm{mg}, 0.75 \mathrm{mmol})$ in $0.5 \mathrm{~mL} \mathrm{DME}, i \mathrm{PrMgCl}(0.89 \mathrm{~mL}, 0.80 \mathrm{mmol}, 0.9 \mathrm{M}$ in THF) is added dropwise via syringe at $-20{ }^{\circ} \mathrm{C}$. The solution is stirred for $3 \mathrm{~h}$, than $\mathrm{CuCl}$ (5.0 $\mathrm{mg}, 10 \mathrm{~mol} \%)$ and $\mathrm{P}(\mathrm{OEt})_{3}(17 \mathrm{mg}, 20 \mathrm{~mol} \%)$ is added. This mixture is added over $30 \mathrm{~min}$ via canula to a prewarmed mixture of diethyl furan-3-ylmethyl phosphate $(117 \mathrm{mg}$, $0.50 \mathrm{mmol})$ and TBAI (19 mg, $10 \mathrm{~mol} \%)$. The reaction mixture is heated at $60{ }^{\circ} \mathrm{C}$ for $1 \mathrm{~h}$. The usual workup and purification by flash chromatography (pentane/ether 49:1) yielded $2 \mathbf{r}$ as colourless solid (165 mg, 92\%).

Mp:. $63^{\circ} \mathrm{C}$.

${ }^{1} \mathbf{H}-\mathbf{N M R}\left(\mathrm{CDCl}_{3}, 600 \mathrm{MHz}, 25^{\circ} \mathrm{C}\right): \delta=7.71(\mathrm{~d}, J=8.1 \mathrm{~Hz}, 1 \mathrm{H}), 7.33-7.13(\mathrm{~m}, 8 \mathrm{H}), 7.01(\mathrm{~d}$, $J=7.4 \mathrm{~Hz}, 2 \mathrm{H}), 6.29$ (s, 1H), 5.78 (s, 2H), 4.33-4.29 (m, 4H), 1.28 (t, J=7.1 Hz, 3H).

${ }^{13} \mathrm{C}-\mathrm{NMR}\left(\mathrm{CDCl}_{3}, 150 \mathrm{MHz}, 25{ }^{\circ} \mathrm{C}\right): \delta=162.4,142.6,139.2,138.8,138.6,128.5,127.0$, 126.7, 126.1, 125.6, 124.5, 123.3, 120.9, 120.4, 111.2, 110.7, 60.61, 48.3, 21.1, 14.2.

IR (KBr): 3063 (w), 2983 (w), 1685(vs), 1522 (m), 1498 (m), 1452 (m), 1372 (m), 1263 (s), 1248 (s), 1192 (m), 1137 (s), 1124 (s), 1025 (m), 1012 (m), 872 (m), 735 (s); 697 (m).

MS (70 eV, EI), m/z (\%): 359 (77, M+), 330 (9), 286 (12), 268 (92), 222 (36), 194 (6), 166 (10), 91 (100), 65 (7).

HRMS $m / z$ : calcd. for $\mathrm{C}_{23} \mathrm{H}_{21} \mathrm{NO}_{3}: 359.1521$; found: 359.1500 . 


\section{Indole derivative $2 r$}

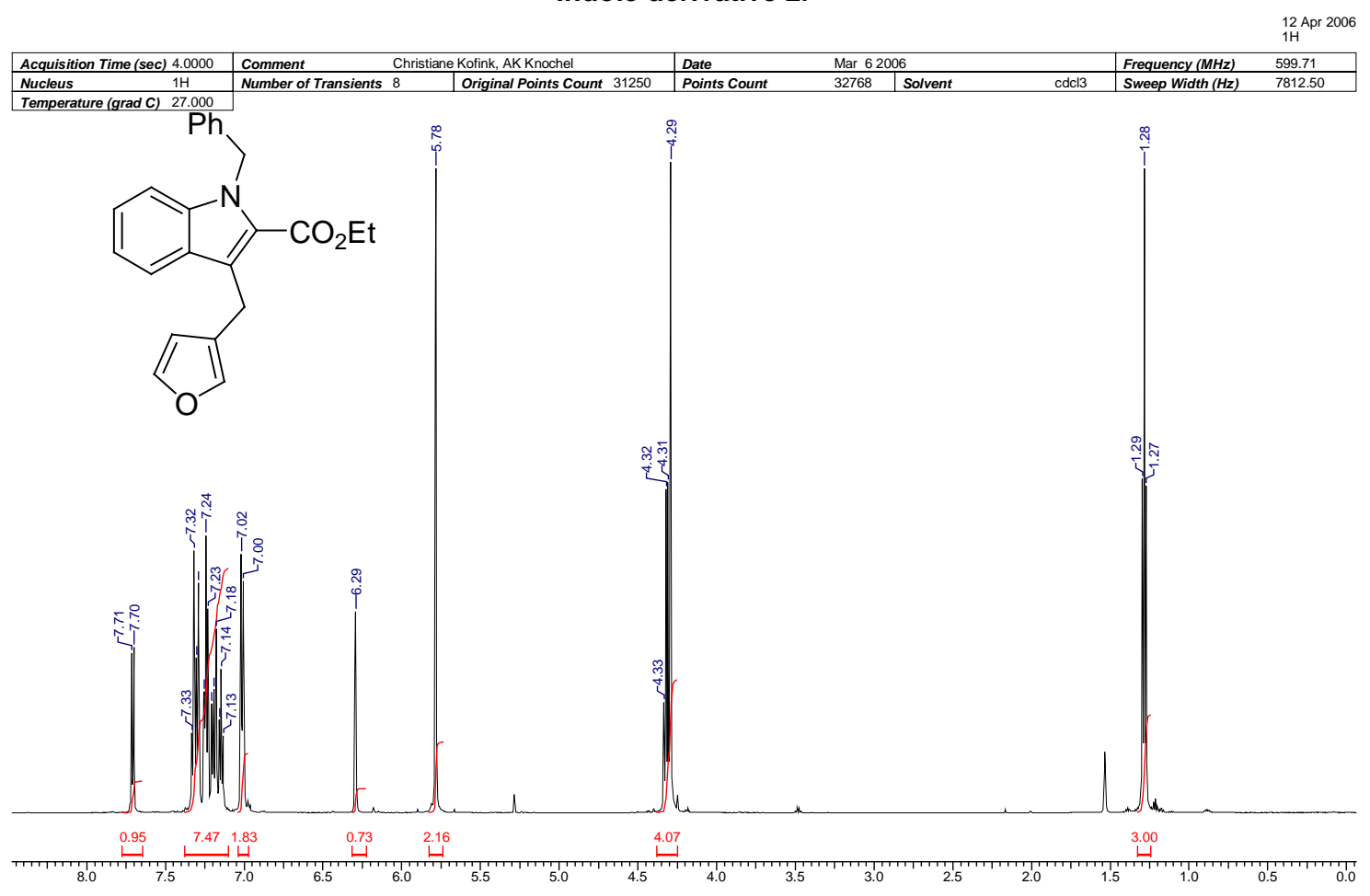

Indole derivative $2 r$
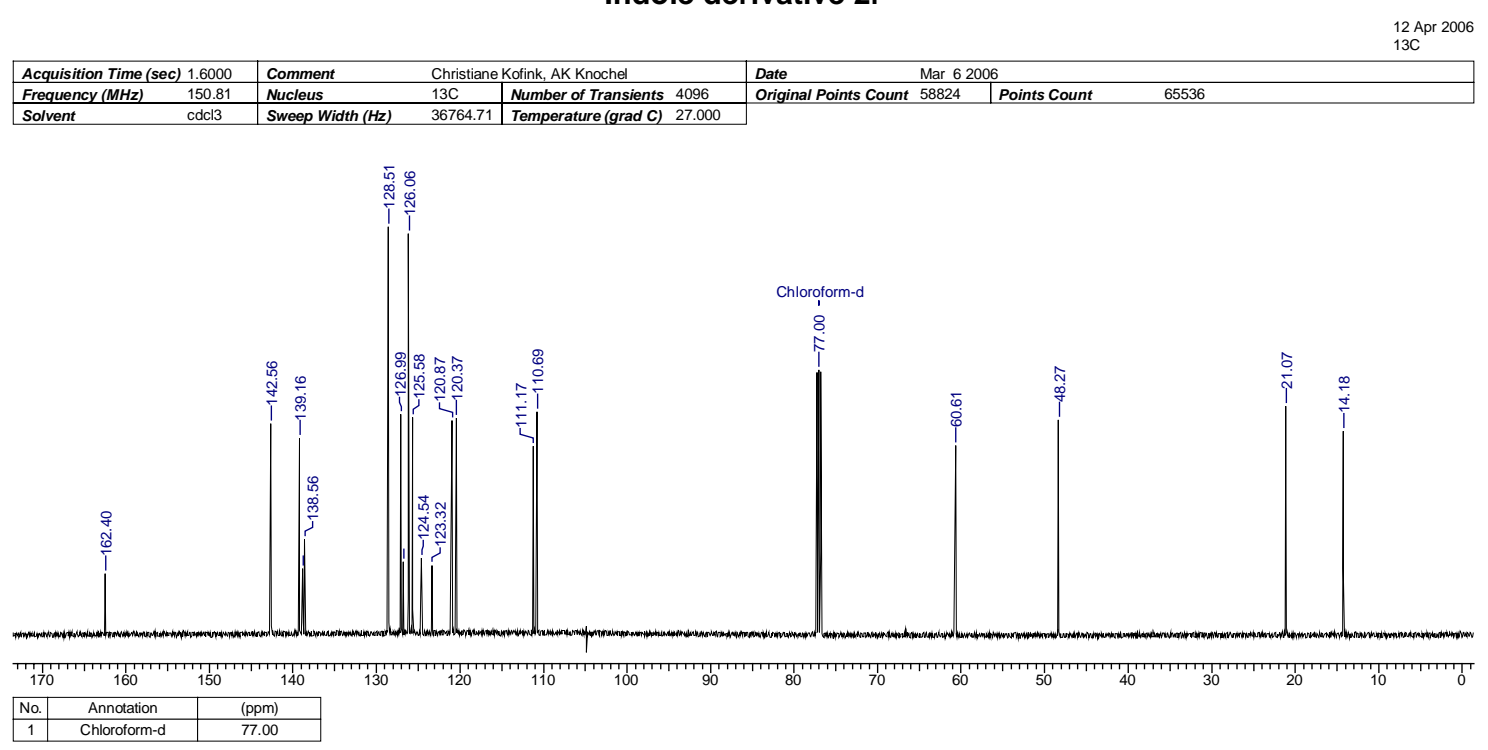


\section{Typical Procedure for the Preparation of Functionalized Phosphates:}

\section{Typical Procedure 3 (TP 3).}

In a round bottom flask with magnetic stirring and a septum, the corresponding benzylic alcohol (1.0 equiv.), DMAP (0.1 equiv.) and $\mathrm{NEt}_{3}$ (1.1 equiv.) are dissolved in THF $(10 \mathrm{~mL} / \mathrm{mmol})$. Then diethyl chlorophosphate (1.1 equiv.) is added slowly via syringe. During the addition a white precipitat forms. The reaction mixture is stirred at $\mathrm{rt}$ until the GC of an aliquot shows completion of the reaction, then quenched with sat. $\mathrm{NH}_{4} \mathrm{Cl}$ solution, extracted with ether, and the product is purified by column chromatography. 


\section{Benzyl diethyl phosphate 4a}

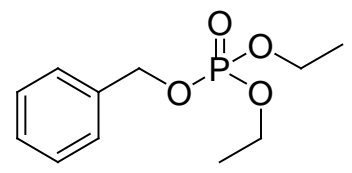

Prepared according to TP 3. Phenylmethanol (15.0 mmol, $1.62 \mathrm{~g}), \mathrm{NEt}_{3}(22.5 \mathrm{mmol}, 3.16 \mathrm{~mL})$ and DMAP (1.50 mmol, $183 \mathrm{mg})$, are dissolved in THF. This mixture is treated with diethyl chlorophosphate (16.5 mmol, $2.85 \mathrm{~g})$ and stirred at $\mathrm{rt}$ for $4 \mathrm{~h}$. Standard work up and purification yielded $\mathbf{4 a}(3.20 \mathrm{~g}, 88 \%)$ as colourless liquid.

${ }^{1} \mathbf{H}-\mathbf{N M R}\left(\mathrm{CDCl}_{3}, 300 \mathrm{MHz}, 25{ }^{\circ} \mathrm{C}\right): \delta=7.30-7.21(\mathrm{~m}, 5 \mathrm{H}), 4.96(\mathrm{~d}, J=8.3 \mathrm{~Hz}, 2 \mathrm{H}), 4.02-$ $3.92(\mathrm{~m}, 4 \mathrm{H}), 1.19(\mathrm{t}, J=7.1 \mathrm{~Hz}, 3 \mathrm{H})$.

${ }^{13} \mathrm{C}-\mathrm{NMR}\left(\mathrm{CDCl}_{3}, 75 \mathrm{MHz}, 25{ }^{\circ} \mathrm{C}\right): \delta=135.70(\mathrm{~d}, J(\mathrm{C}, \mathrm{P})=6.8 \mathrm{~Hz}), 128.1,128.0,127.4$, $68.59(\mathrm{~d}, J(\mathrm{C}, \mathrm{P})=5.6 \mathrm{~Hz}), 63.36(\mathrm{~d}, J(\mathrm{C}, \mathrm{P})=5.9 \mathrm{~Hz}), 15.65(\mathrm{~d}, J(\mathrm{C}, \mathrm{P})=6.8 \mathrm{~Hz})$.

${ }^{31} \mathbf{P}-\mathbf{N M R}\left(\mathrm{CDCl}_{3}, 200 \mathrm{MHz}, 25^{\circ} \mathrm{C}\right): \delta=0.084$.

IR (KBr): 2980 (w), 1518 (w), 1260 (s), 1025 (vs), 1009 (vs), 801 (m), 751 (w).

MS (70 eV, EI), m/z (\%): $244\left(51, \mathrm{M}^{+}\right), 215$ (25), 187 (32), 138 (17),107 (95), 91 (100), 65 (17).

HRMS $m / z$ : calcd. for $\mathrm{C}_{11} \mathrm{H}_{17} \mathrm{O}_{4} \mathrm{P}: 244.0864$; found: 244.0879 . 


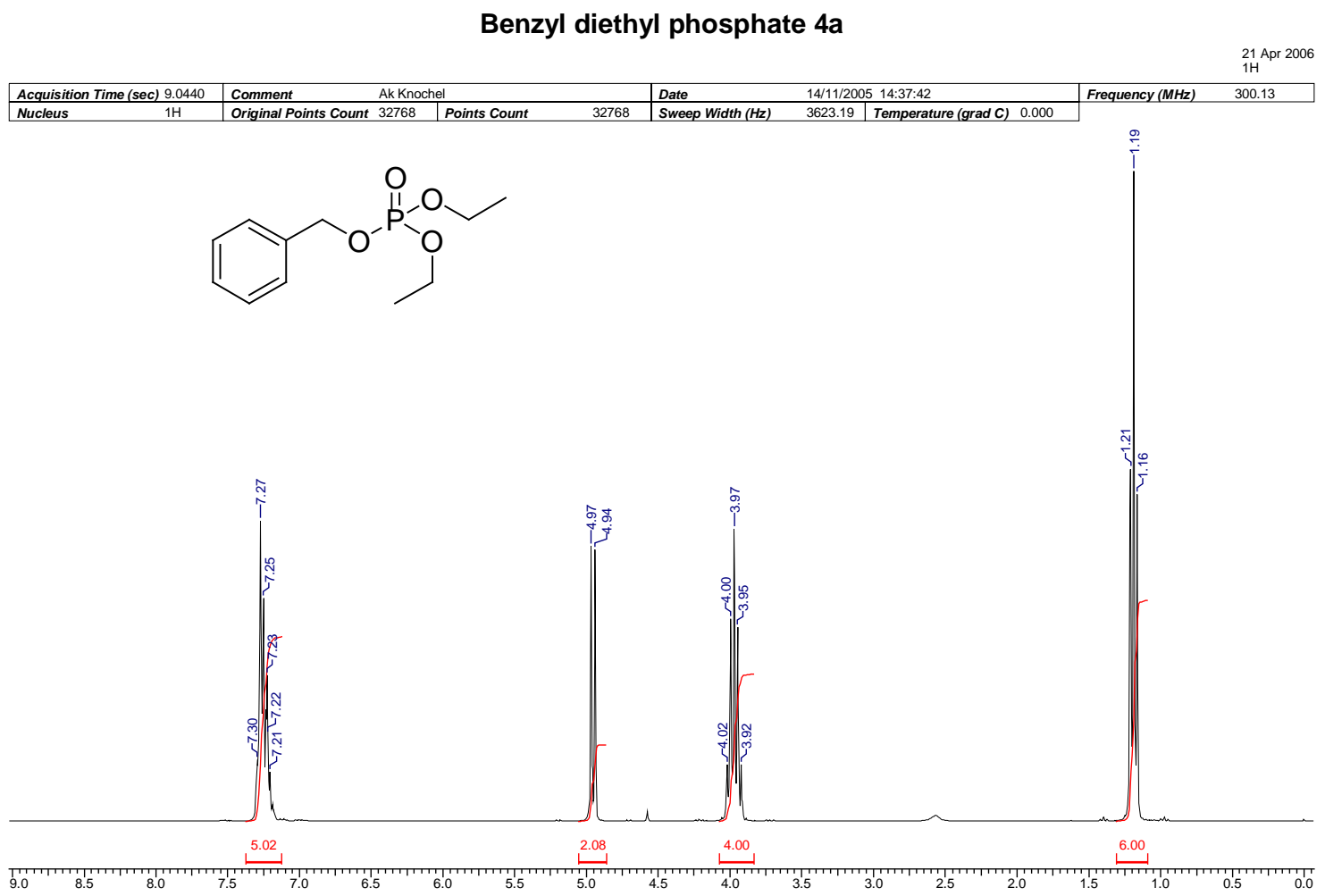

\section{Benzyl diethyl phosphate 4a}

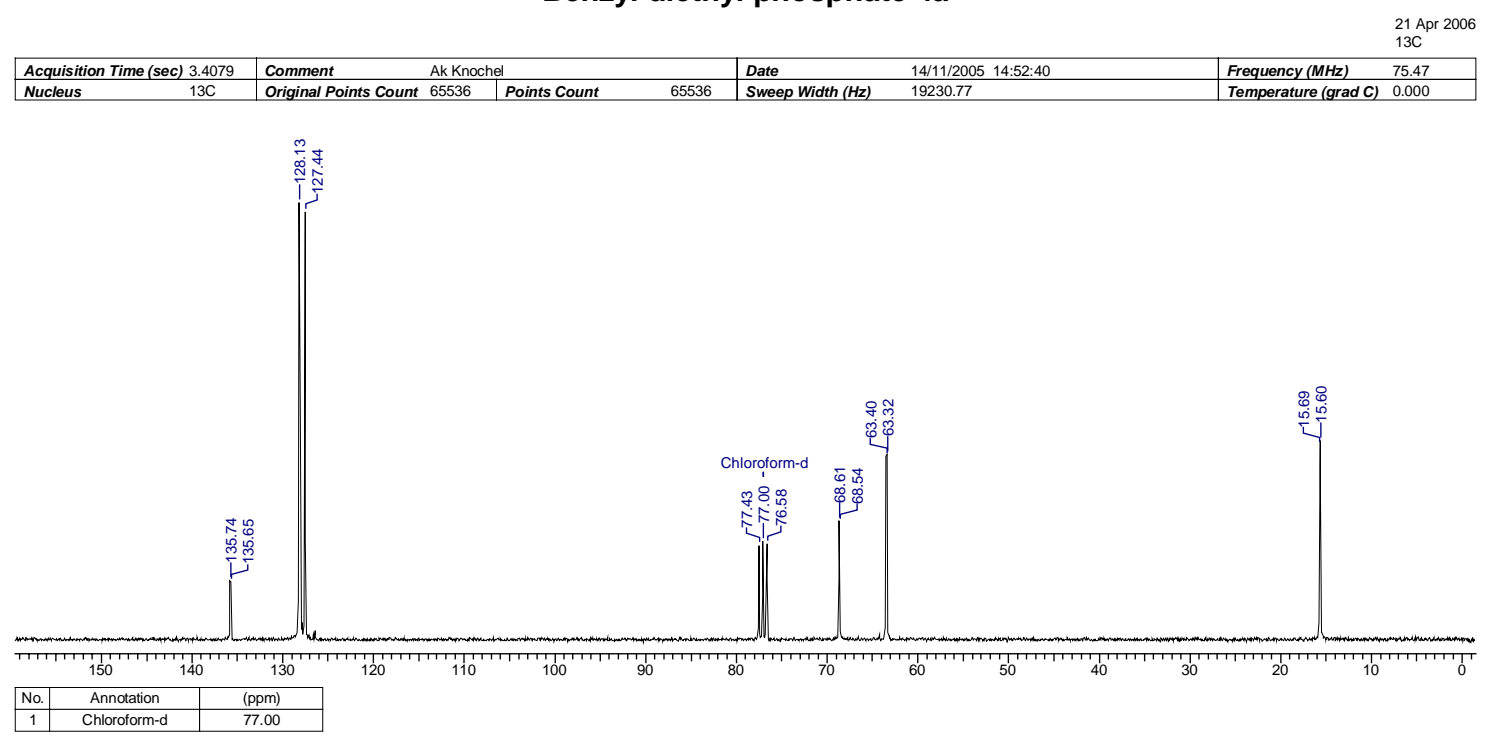




\section{Diethyl 4-methylbenzyl phosphate 4b}

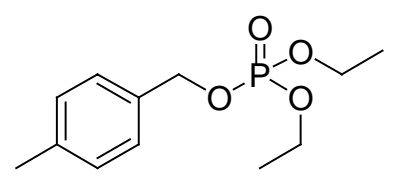

Prepared according to TP 3. p-Tolylmethanol (5.00 mmol, $611 \mathrm{mg}), \mathrm{NEt}_{3}(5.5 \mathrm{mmol}$, $0.77 \mathrm{~mL})$ and DMAP $(0.10 \mathrm{mmol}, 61 \mathrm{mg})$, are dissolved in THF. This mixture is treated with diethyl chlorophosphate $(5.50 \mathrm{mmol}, 949 \mathrm{mg})$ and stirred at $\mathrm{rt}$ for $4 \mathrm{~h}$. Standard work up and purification yielded $\mathbf{4 b}(1.07 \mathrm{~g}, 83 \%)$ as colourless liquid.

${ }^{1}$ H-NMR $\left(\mathrm{CDCl}_{3}, 300 \mathrm{MHz}, 25{ }^{\circ} \mathrm{C}\right): \delta=7.20(\mathrm{~d}, J=8.1 \mathrm{~Hz}, 2 \mathrm{H}), 7.09(\mathrm{~d}, J=8.0 \mathrm{~Hz}, 2 \mathrm{H})$, $4.94(\mathrm{~d}, J=8.1 \mathrm{~Hz}, 2 \mathrm{H}), 4.05-3.94(\mathrm{~m}, 4 \mathrm{H}), 2.27$ (s, 3H), 1.22 (t, $J=7.1 \mathrm{~Hz}, 6 \mathrm{H})$.

${ }^{13} \mathrm{C}-\mathrm{NMR}\left(\mathrm{CDCl}_{3}, 75 \mathrm{MHz}, 25^{\circ} \mathrm{C}\right): \delta=138.2,132.8(\mathrm{~d}, J(\mathrm{C}, \mathrm{P})=6.8 \mathrm{~Hz}), 129.1,127.9,68.83$ $(\mathrm{d}, J(\mathrm{C}, \mathrm{P})=5.6 \mathrm{~Hz}), 63.57(\mathrm{~d}, J(\mathrm{C}, \mathrm{P})=5.9 \mathrm{~Hz}), 21.03,15.92(\mathrm{~d}, J(\mathrm{C}, \mathrm{P})=6.8 \mathrm{~Hz})$.

${ }^{31} \mathbf{P}$-NMR $\left(\mathrm{CDCl}_{3}, 200 \mathrm{MHz}, 25{ }^{\circ} \mathrm{C}\right): \delta=0.159$.

IR (KBr): 2983 (w), 1518 (w), 1262 (s), 1025 (vs), 1009 (vs), 801 (m), 753 (w).

MS (70 eV, EI), m/z (\%): 258 (45, M+), 229 (44), 132 (21), 121 (80), 105 (100), 77 (22).

HRMS $m / z$ : calcd. for $\mathrm{C}_{12} \mathrm{H}_{19} \mathrm{O}_{4} \mathrm{P}: 258.1021$; found: 258.1001 . 
Diethyl 4-methylbenzyl phosphate 4b

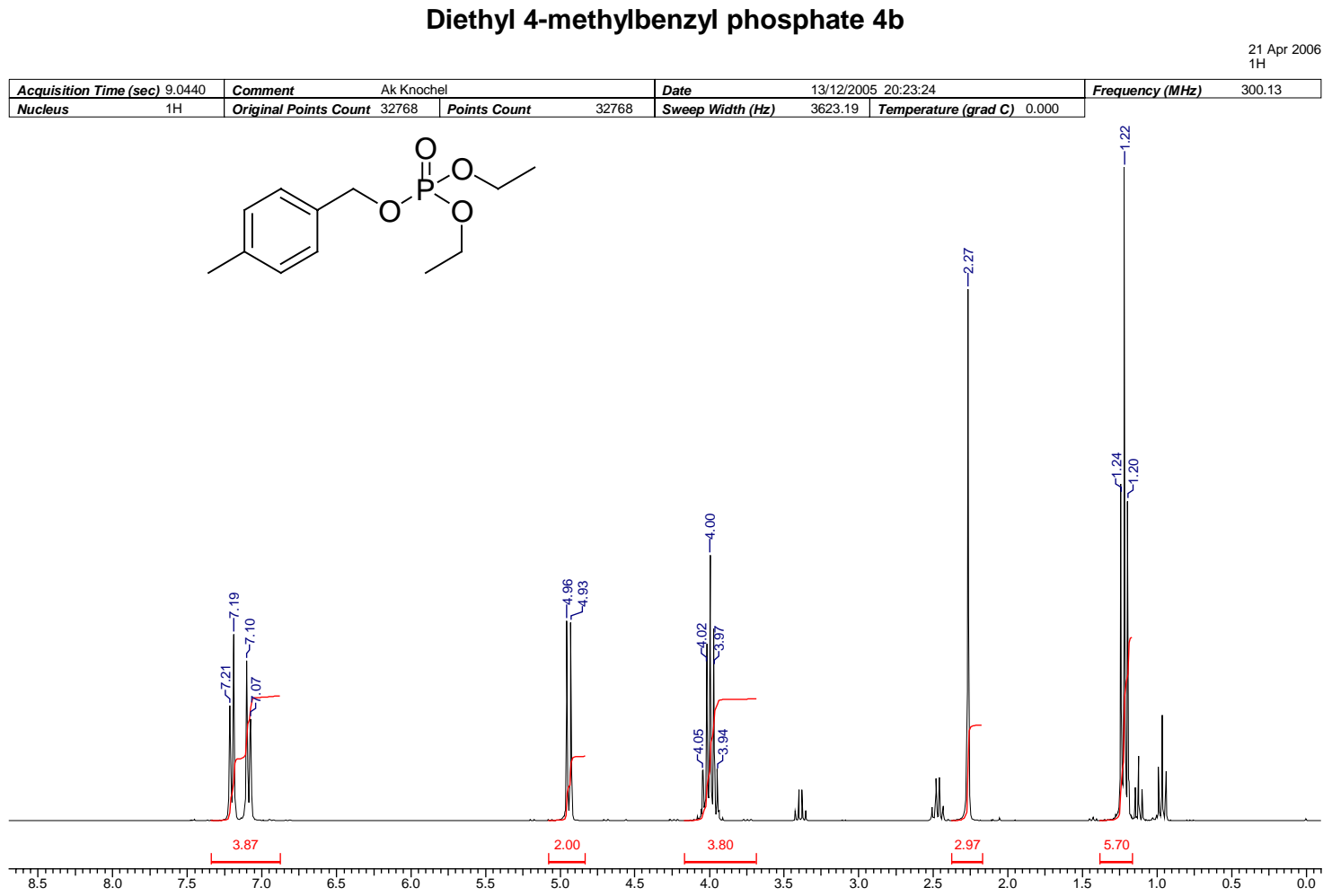

\section{Diethyl 4-methylbenzyl phosphate 4b}

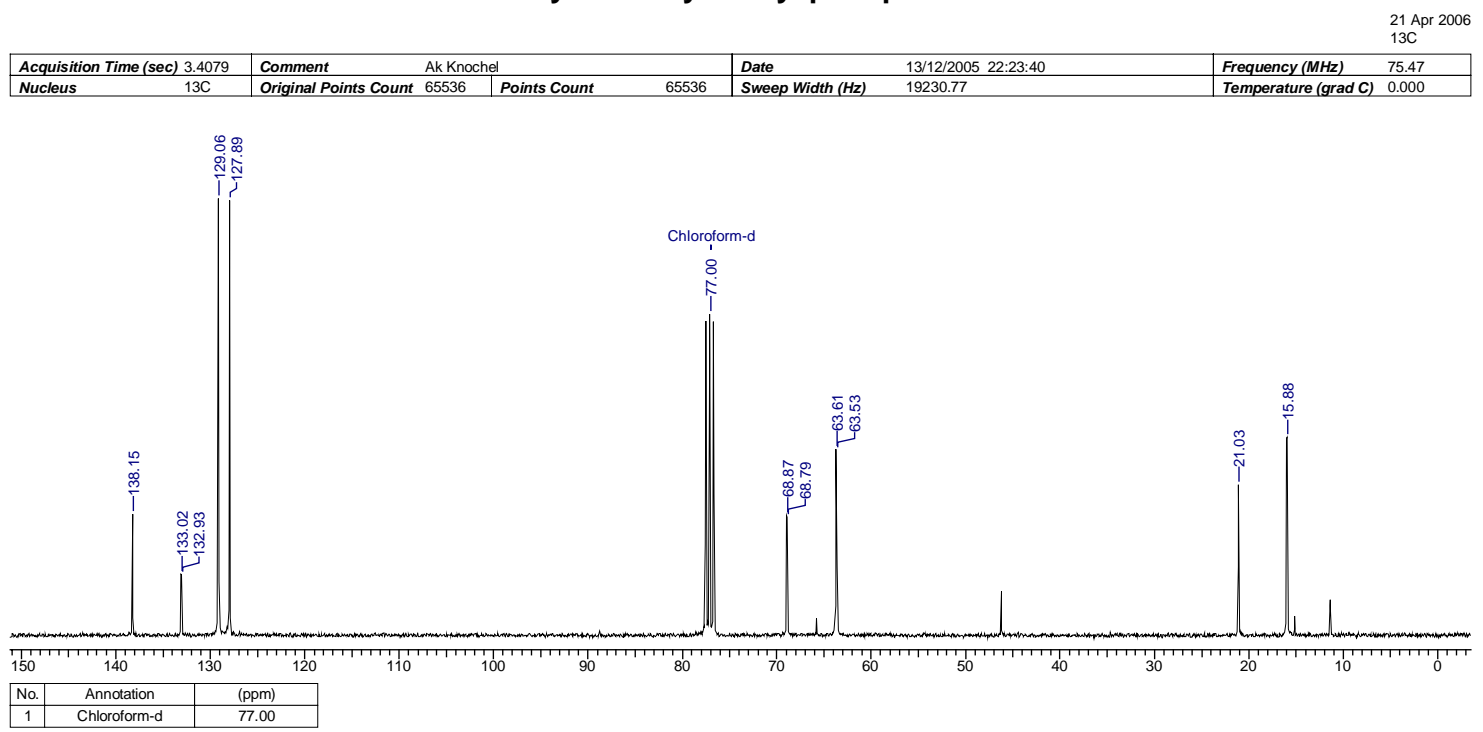




\section{4-Bromobenzyl diethyl phosphate 4c}

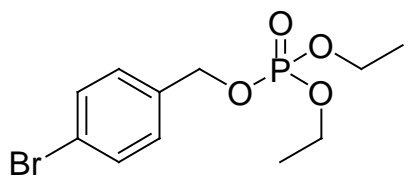

Prepared according to TP 3. (4-Bromophenyl)methanol (5.00 mmol, $935 \mathrm{mg}$ ), $\mathrm{NEt}_{3}$ $(5.5 \mathrm{mmol}, 0.77 \mathrm{~mL})$ and DMAP $(0.10 \mathrm{mmol}, 61 \mathrm{mg})$, are dissolved in THF. This mixture is treated with diethyl chlorophosphate $(5.50 \mathrm{mmol}, 949 \mathrm{mg})$ and stirred at $\mathrm{rt}$ for $4 \mathrm{~h}$. Standard work up and purification yielded $\mathbf{4 c}(1.27 \mathrm{~g}, 78 \%)$ as colourless liquid.

${ }^{1} \mathbf{H}$-NMR $\left(\mathrm{CDCl}_{3}, 300 \mathrm{MHz}, 25{ }^{\circ} \mathrm{C}\right): \delta=7.41(\mathrm{~d}, J=8.5 \mathrm{~Hz}, 2 \mathrm{H}), 7.19(\mathrm{~d}, J=8.4 \mathrm{~Hz}, 2 \mathrm{H})$, $4.93(\mathrm{~d}, J=8.3 \mathrm{~Hz}, 2 \mathrm{H}), 4.06-3.96(\mathrm{~m}, 4 \mathrm{H}), 1.23(\mathrm{t}, J=7.1 \mathrm{~Hz}, 6 \mathrm{H})$.

${ }^{13} \mathrm{C}-\mathrm{NMR}\left(\mathrm{CDCl}_{3}, 75 \mathrm{MHz}, 25{ }^{\circ} \mathrm{C}\right): \delta=134.95(\mathrm{~d}, J(\mathrm{C}, \mathrm{P})=6.8 \mathrm{~Hz}), 131.5,129.3,122.2$, $67.91(\mathrm{~d}, J(\mathrm{C}, \mathrm{P})=5.3 \mathrm{~Hz}), 63.68(\mathrm{~d}, J(\mathrm{C}, \mathrm{P})=5.9 \mathrm{~Hz}), 15.83(\mathrm{~d}, J(\mathrm{C}, \mathrm{P})=6.8 \mathrm{~Hz})$.

${ }^{31} \mathbf{P}-\mathbf{N M R}\left(\mathrm{CDCl}_{3}, 200 \mathrm{MHz}, 25{ }^{\circ} \mathrm{C}\right): \delta=0.107$.

IR (KBr): 2983 (w), 1595 (w), 1489 (m), 1262 (s), 1020 (vs), 1002 (vs), 973 (s), 852 (w), 798 (m).

MS (70 eV, EI), m/z (\%): $321\left(51, \mathrm{M}^{+}\right), 293$ (40), 265 (46), 185 (100), 171 (96), 125 (45), 89 (70), 63 (16).

HRMS $m / z$ : calcd. for $\mathrm{C}_{11} \mathrm{H}_{16} \mathrm{BrO}_{4} \mathrm{P}: 321.9970$; found: 321.9947 . 


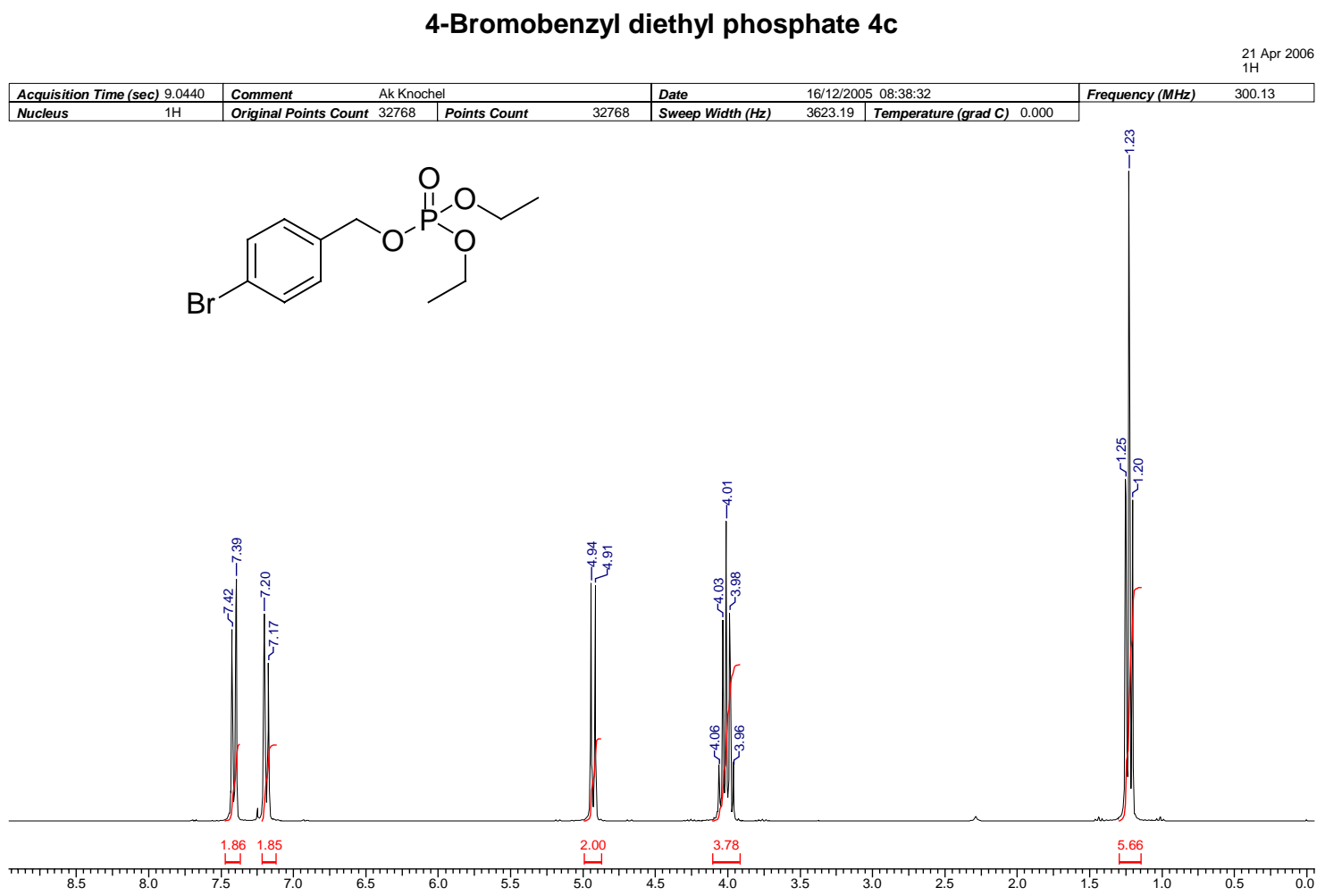

\section{4-Bromobenzyl diethyl phosphate 4c}

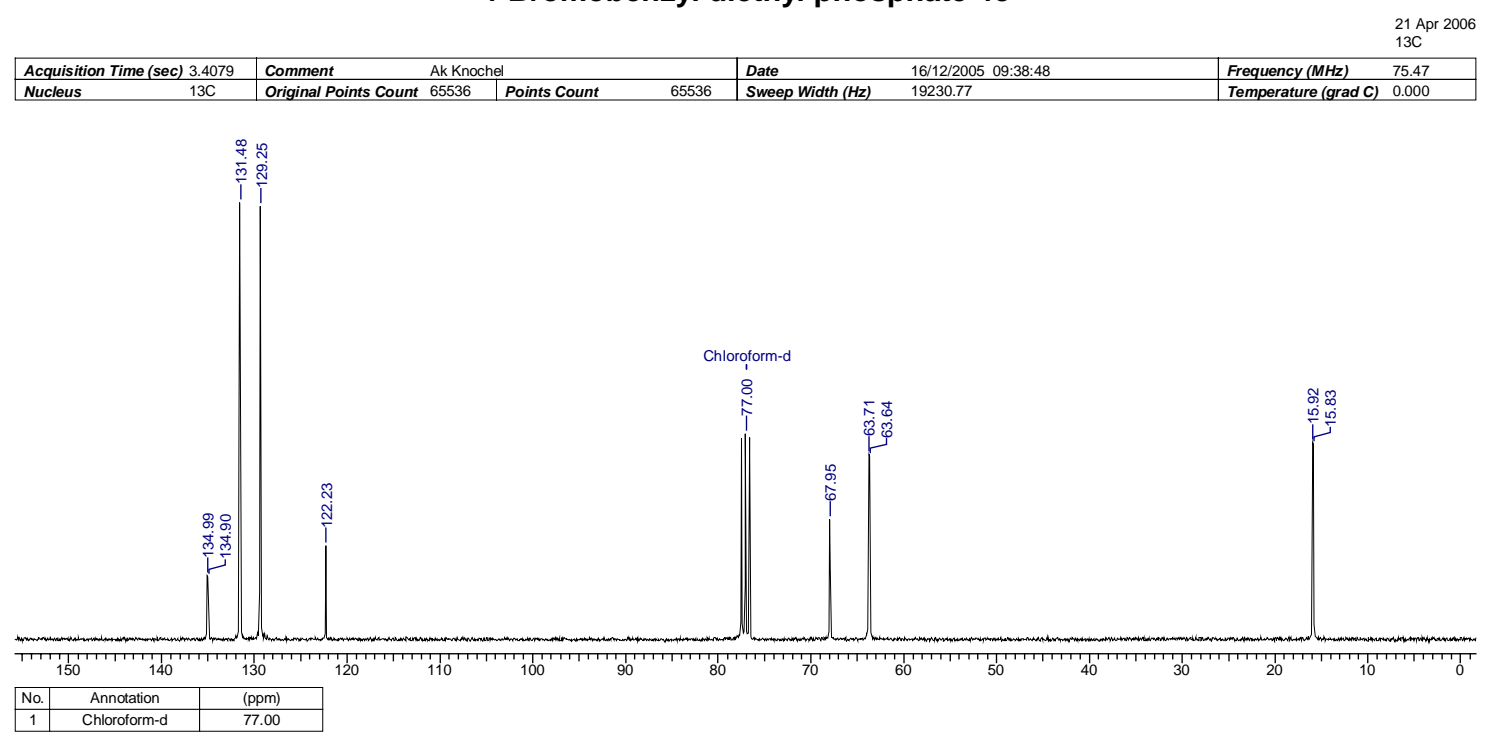




\section{Diethyl 2-methoxybenzyl phosphate 4d}

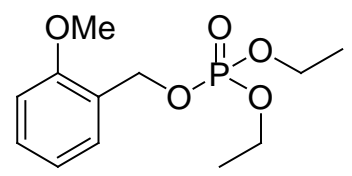

Prepared according to TP 3. (2-Methoxyphenyl)methanol (5.00 mmol, $691 \mathrm{mg}$ ), $\mathrm{NEt}_{3}$ $(5.5 \mathrm{mmol}, 0.77 \mathrm{~mL})$ and DMAP $(0.10 \mathrm{mmol}, 61 \mathrm{mg})$, are dissolved in THF. This mixture is treated with diethyl chlorophosphate $(5.50 \mathrm{mmol}, 949 \mathrm{mg})$ and stirred at $\mathrm{rt}$ for $4 \mathrm{~h}$. Standard work up and purification yielded $\mathbf{4 d}(1.96 \mathrm{~g}, 80 \%)$ as colourless liquid.

${ }^{1} \mathbf{H}$-NMR $\left(\mathrm{CDCl}_{3}, 300 \mathrm{MHz}, 25{ }^{\circ} \mathrm{C}\right): \delta=7.33(\mathrm{~d}, J=7.5 \mathrm{~Hz}, 1 \mathrm{H}), 7.20-7.05(\mathrm{~m}, 1 \mathrm{H}), 6.90$ $6.78(\mathrm{~m}, 2 \mathrm{H}), 5.05(\mathrm{~d}, J=7.2 \mathrm{~Hz}, 2 \mathrm{H}), 4.07-3.98(\mathrm{~m}, 4 \mathrm{H}), 3.75(\mathrm{~s}, 3 \mathrm{H}), 1.23(\mathrm{t}, J=7.1 \mathrm{~Hz}$, $6 \mathrm{H})$.

${ }^{13} \mathrm{C}-\mathrm{NMR}\left(\mathrm{CDCl}_{3}, 75 \mathrm{MHz}, 25{ }^{\circ} \mathrm{C}\right): \delta=156.9,129.5,129.0,124.39(\mathrm{~d}, J(\mathrm{C}, \mathrm{P})=7.6 \mathrm{~Hz})$, $120.3,110.1,64.31(\mathrm{~d}, J(\mathrm{C}, \mathrm{P})=5.0 \mathrm{~Hz}), 63.53(\mathrm{~d}, J(\mathrm{C}, \mathrm{P})=5.9 \mathrm{~Hz}), 55.2,15.93(\mathrm{~d}, J(\mathrm{C}, \mathrm{P})=$ $6.8 \mathrm{~Hz})$.

${ }^{31} \mathbf{P}-\mathrm{NMR}\left(\mathrm{CDCl}_{3}, 200 \mathrm{MHz}, 25^{\circ} \mathrm{C}\right): \delta=0.264$.

IR (KBr): 2983 (w), 1595 (w), 1605 (w), 1495 (m), 1441 (w), 1246 (s), 1021 (vs), 1000 (vs), $964(\mathrm{~s}), 880(\mathrm{w}), 752(\mathrm{~m})$.

MS (70 eV, EI), m/z (\%): 274 (10, M+), 245 (7), 185 (5), 137 (34), 120 (100), 91 (65), 65 (9).

HRMS $m / z$ : calcd. for $\mathrm{C}_{12} \mathrm{H}_{19} \mathrm{O}_{5} \mathrm{P}: 274.097$; found: 274.0985 . 


\section{Diethyl 2-methoxybenzyl phosphate 4d}

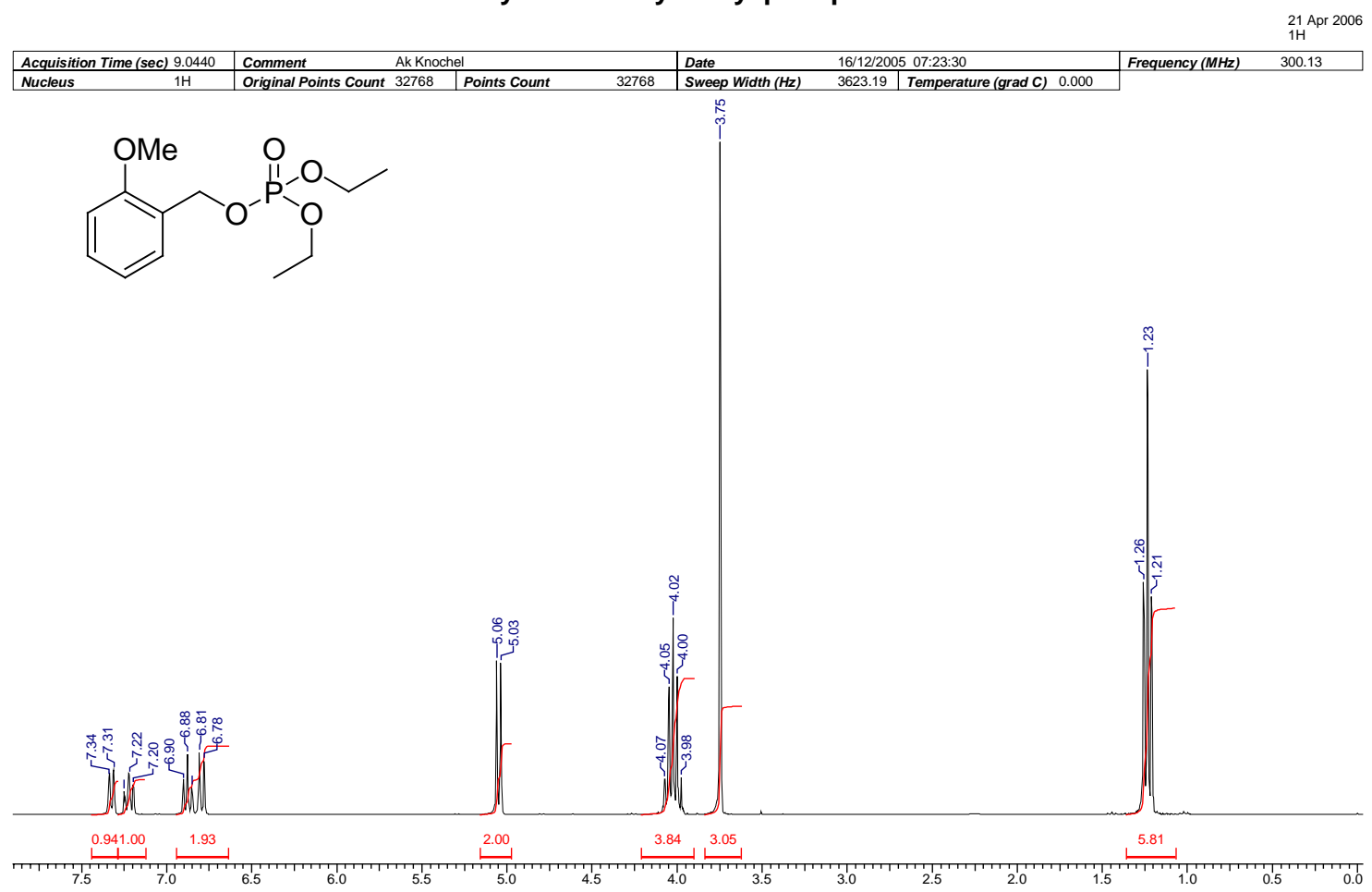

\section{Diethyl 2-methoxybenzyl phosphate 4d}

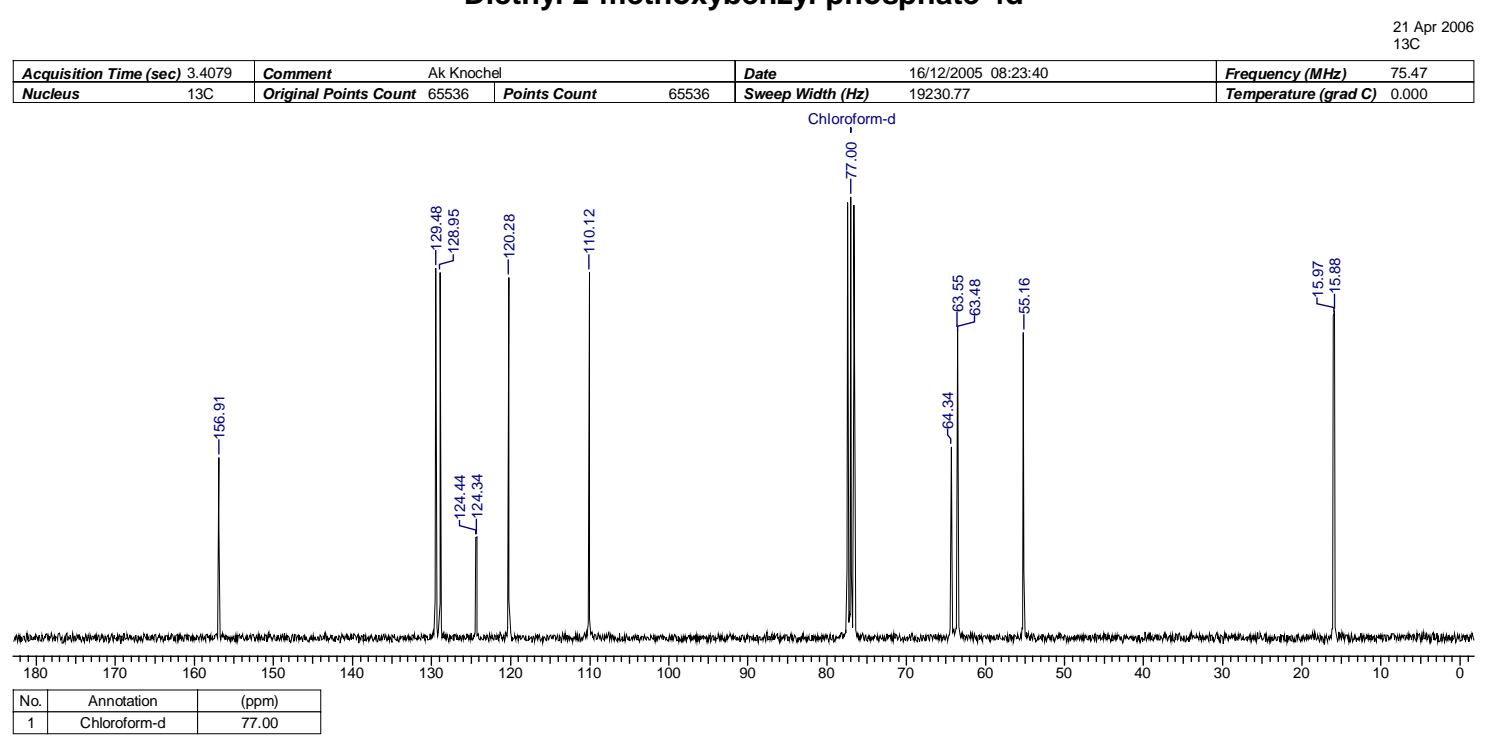




\section{Diethyl 4-methoxybenzyl phosphate 4e}

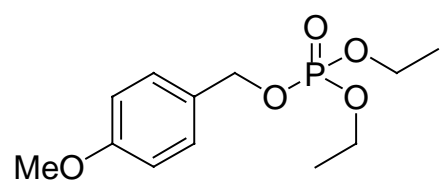

Prepared according to TP 3. (4-Methoxyphenyl)methanol (15.0 mmol, $2.07 \mathrm{~g}$ ), $\mathrm{NEt}_{3}$ (22.5 mmol, $3.16 \mathrm{~mL})$ and DMAP (1.50 mmol, $183 \mathrm{mg})$, are dissolved in THF. This mixture is treated with diethyl chlorophosphate $(16.5 \mathrm{mmol}, 2.83 \mathrm{~g})$ and stirred at $\mathrm{rt}$ for $4 \mathrm{~h}$. Standard work up and purification yielded $\mathbf{4 e}(2.88 \mathrm{~g}, 70 \%)$ as colourless liquid.

${ }^{1}$ H-NMR $\left(\mathrm{CDCl}_{3}, 300 \mathrm{MHz}, 25{ }^{\circ} \mathrm{C}\right): \delta=7.22(\mathrm{~d}, J=8.7 \mathrm{~Hz}, 2 \mathrm{H}), 6.78(\mathrm{~d}, J=8.7 \mathrm{~Hz}, 2 \mathrm{H})$, 4.88 (d, J= 8.4 Hz, 2H), 3.99-3.90 (m, 4H), 3.67 (s, 3H), 1.18 (t, $J=7.1 \mathrm{~Hz}, 6 \mathrm{H})$.

${ }^{13} \mathrm{C}-\mathrm{NMR}\left(\mathrm{CDCl}_{3}, 75 \mathrm{MHz}, 25{ }^{\circ} \mathrm{C}\right): \delta=159.5,129.4,127.76(\mathrm{~d}, J(\mathrm{C}, \mathrm{P})=6.5 \mathrm{~Hz}), 113.5$, $68.51(\mathrm{~d}, J(\mathrm{C}, \mathrm{P})=5.9 \mathrm{~Hz}), 63.26(\mathrm{~d}, J(\mathrm{C}, \mathrm{P})=5.9 \mathrm{~Hz}), 54.8,15.61(\mathrm{~d}, J(\mathrm{C}, \mathrm{P})=6.8 \mathrm{~Hz})$.

${ }^{31} \mathbf{P}$-NMR $\left(\mathrm{CDCl}_{3}, 200 \mathrm{MHz}, 25{ }^{\circ} \mathrm{C}\right): \delta=0.264$.

IR (KBr): 2983 (w), 1595 (w), 1605 (w), 1495 (m), 1441 (w), 1246 (s), 1021 (vs), 1000 (vs), $964(\mathrm{~s}), 880(\mathrm{w}), 752(\mathrm{~m})$.

MS (70 eV , EI), m/z (\%): $274\left(10, \mathrm{M}^{+}\right), 245$ (7), 185 (5), 137 (34), 120 (100), 91 (65), 65 (9).

HRMS $m / z$ : calcd. for $\mathrm{C}_{12} \mathrm{H}_{19} \mathrm{O}_{5} \mathrm{P}:$ : 274.097; found: 274.0985 . 


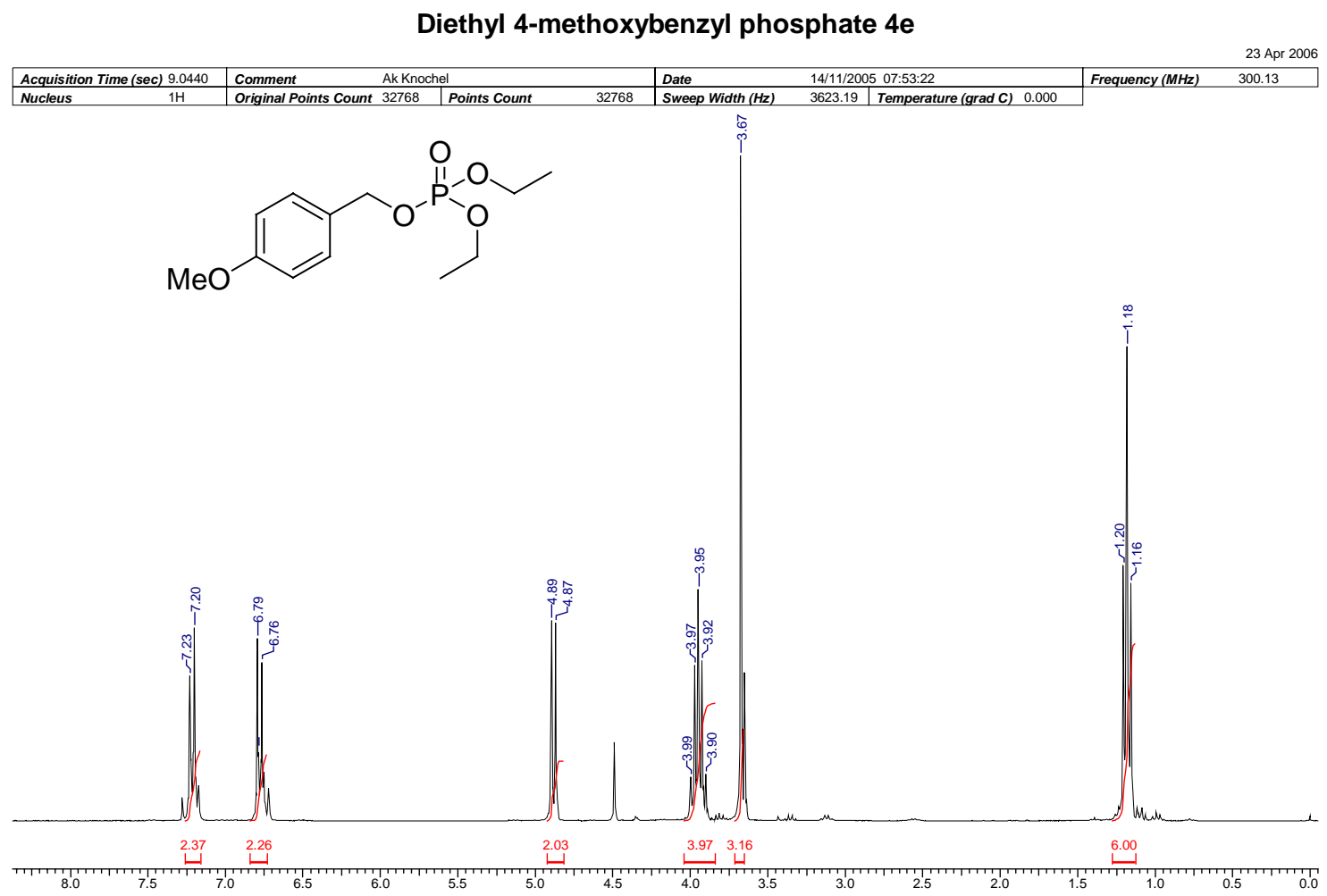

\section{Diethyl 4-methoxybenzyl phosphate 4e}

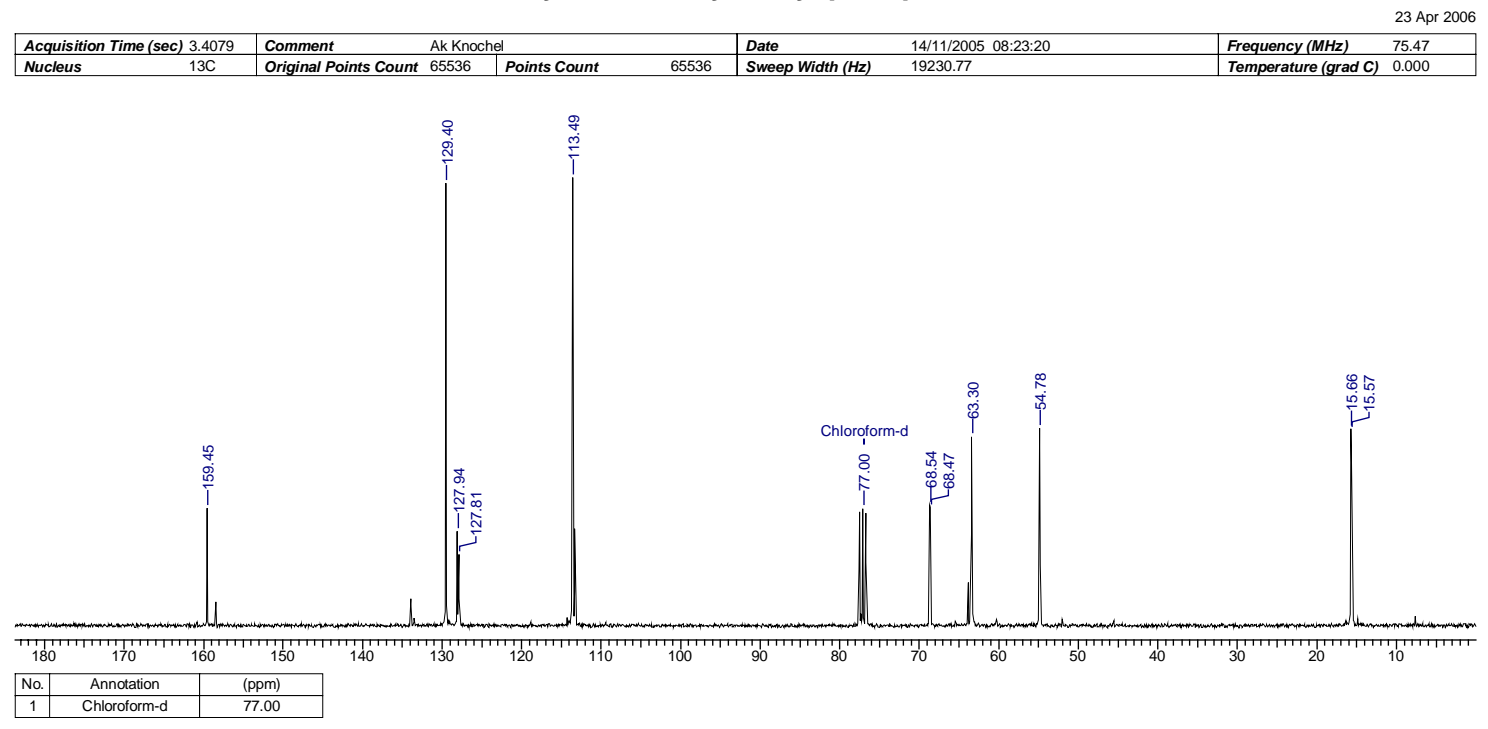




\section{Diethyl furan-3-ylmethyl phosphate $4 f$}

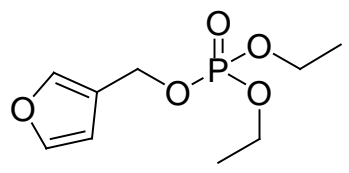

Prepared according to TP 3. Furan-3-ylmethanol (20.0 mmol, $1.96 \mathrm{~g}), \mathrm{NEt}_{3}$ (21.0 mmol, $2.95 \mathrm{~mL}$ ) and DMAP (2.00 mmol, $244 \mathrm{mg}$ ), are dissolved in THF. This mixture is treated with diethyl chlorophosphate (21.0 mmol, $3.62 \mathrm{~g})$ and stirred at $\mathrm{rt}$ for $8 \mathrm{~h}$. Standard work up and purification yielded $\mathbf{4 f}$ (3.69 g, 79\%) as colourless liquid.

${ }^{1} \mathbf{H}-\mathrm{NMR}\left(\mathrm{CDCl}_{3}, 300 \mathrm{MHz}, 25{ }^{\circ} \mathrm{C}\right): \delta=7.49$ (s br, $\left.1 \mathrm{H}\right), 7.40$ (s br, 1H), 6.48 (s br, 1H), 4.96 $(\mathrm{d}, J=8.8 \mathrm{~Hz}, 2 \mathrm{H}), 4.13-4.03(\mathrm{~m}, 4 \mathrm{H}), 1.31(\mathrm{t}, J=7.1 \mathrm{~Hz}, 6 \mathrm{H})$.

${ }^{13} \mathrm{C}-\mathrm{NMR}\left(\mathrm{CDCl}_{3}, 75 \mathrm{MHz}, 25{ }^{\circ} \mathrm{C}\right): \delta=143.5,141.3,120.89(\mathrm{~d}, J(\mathrm{C}, \mathrm{P})=6.1 \mathrm{~Hz}), 110.2$, $63.68(\mathrm{~d}, J(\mathrm{C}, \mathrm{P})=5.5 \mathrm{~Hz}), 60.63(\mathrm{~d}, J(\mathrm{C}, \mathrm{P})=5.5 \mathrm{~Hz}), 15.99(\mathrm{~d}, J(\mathrm{C}, \mathrm{P})=6.6 \mathrm{~Hz})$.

${ }^{31} \mathbf{P}$-NMR $\left(\mathrm{CDCl}_{3}, 200 \mathrm{MHz}, 25{ }^{\circ} \mathrm{C}\right): \delta=0.253$.

IR (KBr): 2984 (w), 1505 (w), 1444 (w), 1259 (s), 1160 (m), 1021 (vs), 972 (vs), 873 (s), 798 (s), $739(\mathrm{~m})$.

MS (70 eV, EI), m/z (\%): 234 (36, M+), 206 (18), 109 (27), 97 (67), 81 (100), 53 (19).

HRMS $m / z$ : calcd. for $\mathrm{C}_{9} \mathrm{H}_{15} \mathrm{O}_{5} \mathrm{P}: 234.0657$; found: 234.0661 . 


\section{Diethyl furan-3-ylmethyl phosphate 4f}

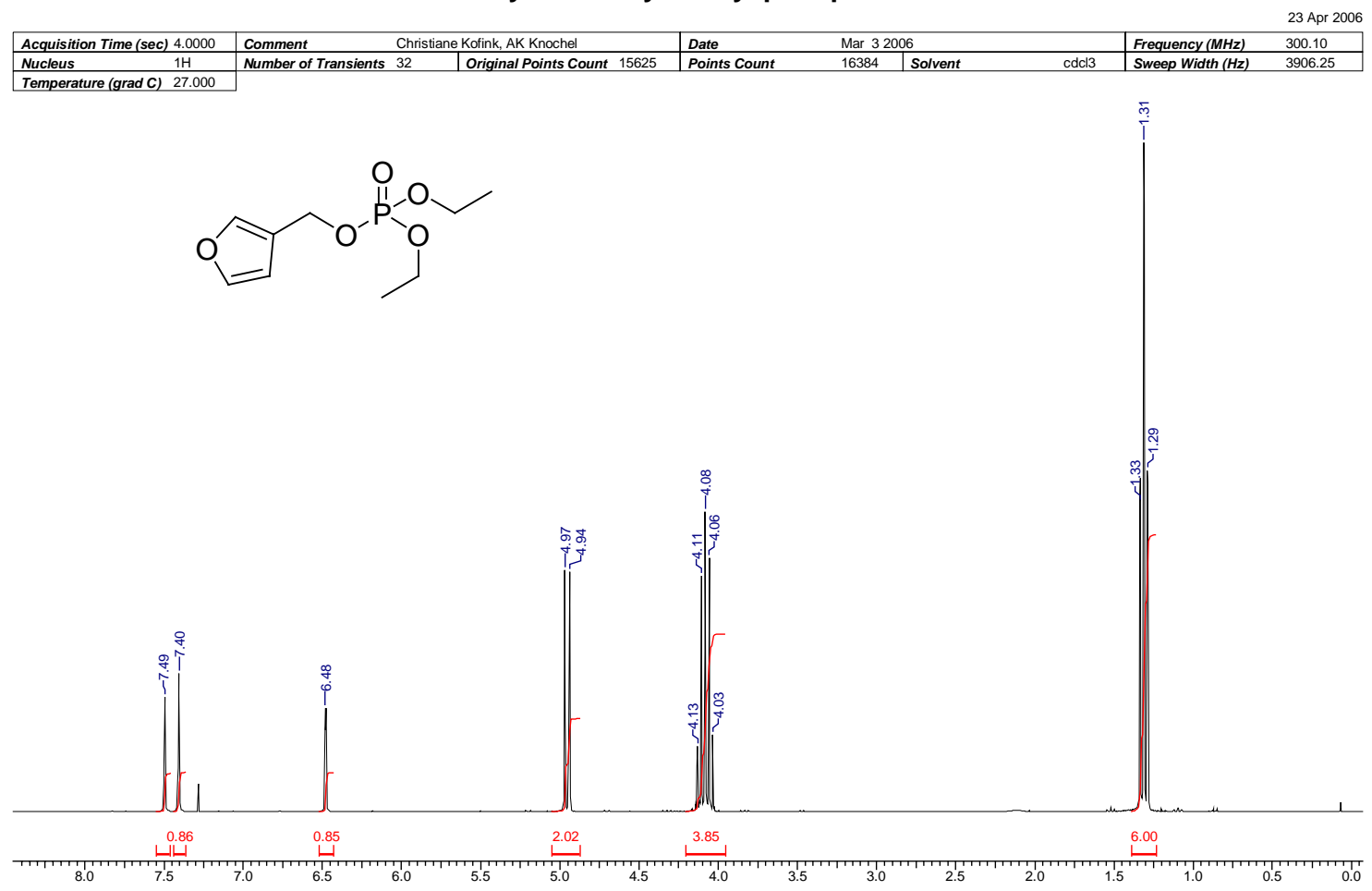

\section{Diethyl furan-3-ylmethyl phosphate $4 f$}

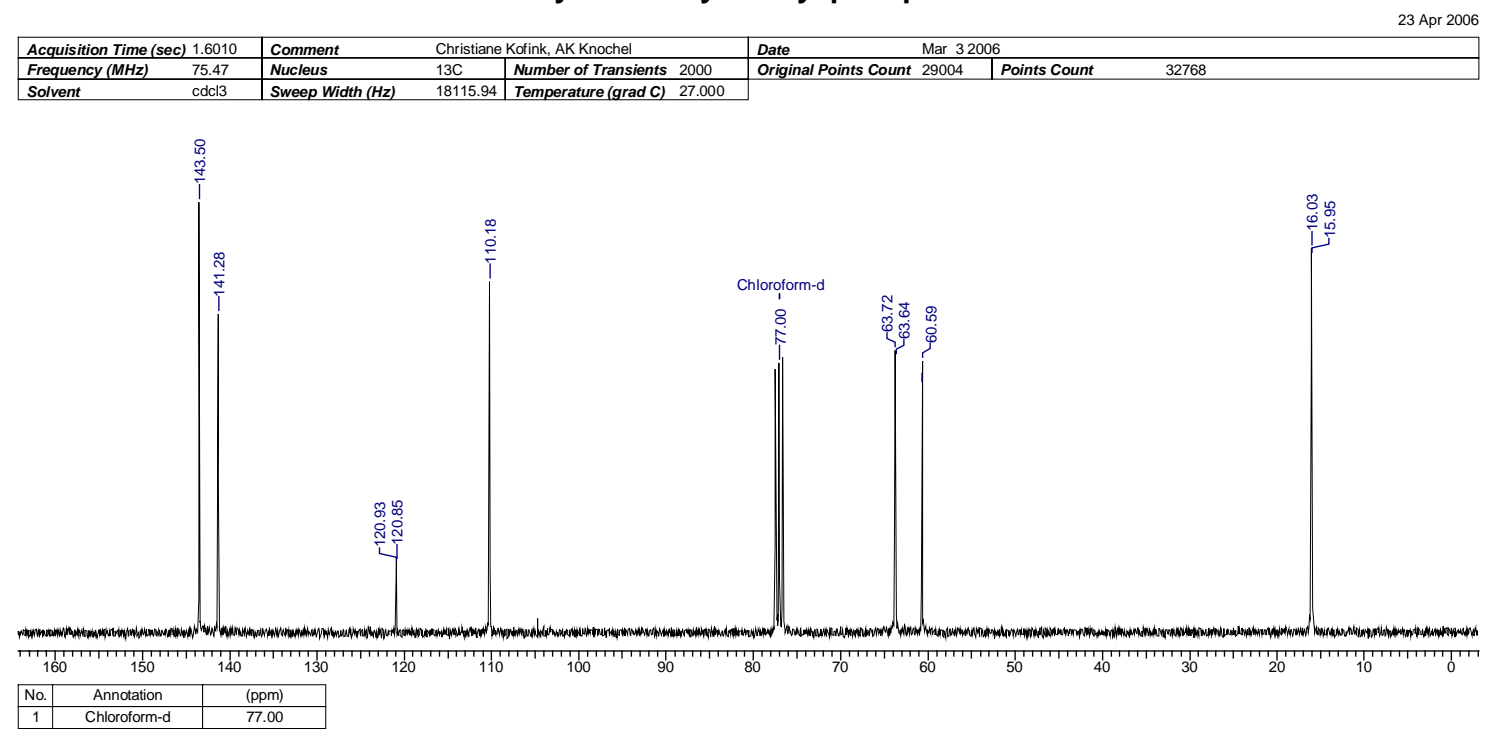




\section{Diethyl thiophen-2-ylmethyl phosphate 4g}

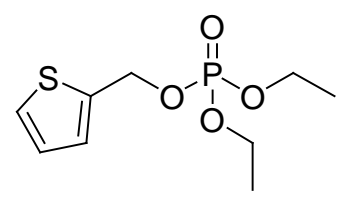

Prepared according to TP 3. Thiophen-2-ylmethanol (20.0 mmol, $2.88 \mathrm{~g}), \mathrm{NEt}_{3}(21.0 \mathrm{mmol}$ $2.95 \mathrm{~mL}$ ) and DMAP (2.00 mmol, $244 \mathrm{mg}$ ), are dissolved in THF. This mixture is treated with diethyl chlorophosphate $(21.0 \mathrm{mmol}, 3.62 \mathrm{~g})$ and stirred at $\mathrm{rt}$ for $8 \mathrm{~h}$. Standard work up and purification yielded $\mathbf{4 g}(2.68 \mathrm{~g}, 54 \%)$ as colourless liquid.

${ }^{1} \mathbf{H}$-NMR $\left(\mathrm{CDCl}_{3}, 300 \mathrm{MHz}, 25^{\circ} \mathrm{C}\right): \delta=7.29$ (s br, $\left.1 \mathrm{H}\right), 7.07$ (s br, 1H), 6.95 (s br, 1H), 5.18 $(\mathrm{d}, J=8.8 \mathrm{~Hz}, 2 \mathrm{H}), 4.09-4.00(\mathrm{~m}, 4 \mathrm{H}), 1.26(\mathrm{t}, J=7.1 \mathrm{~Hz}, 6 \mathrm{H})$.

${ }^{13} \mathrm{C}-\mathrm{NMR}\left(\mathrm{CDCl}_{3}, 75 \mathrm{MHz}, 25{ }^{\circ} \mathrm{C}\right): \delta=138.04(\mathrm{~d}, J(\mathrm{C}, \mathrm{P})=6.6 \mathrm{~Hz}), 127.9,126.9,126.7$, $63.64(\mathrm{~d}, J(\mathrm{C}, \mathrm{P})=5.5 \mathrm{~Hz}), 63.19(\mathrm{~d}, J(\mathrm{C}, \mathrm{P})=5.5 \mathrm{~Hz}), 15.85(\mathrm{~d}, J(\mathrm{C}, \mathrm{P})=6.6 \mathrm{~Hz})$.

${ }^{31} \mathbf{P}-\mathbf{N M R}\left(\mathrm{CDCl}_{3}, 200 \mathrm{MHz}, 25^{\circ} \mathrm{C}\right): \delta=-0.222$.

IR (KBr): 2983 (w), 1505 (w), 1442 (w), 1259 (s), 1167 (m), 994 (vs), 851 (s), 828 (s), 704 (s).

MS (70 eV, EI), m/z (\%): 250 (49, M+), 221 (35), 193 (32), 113 (53), 97 (100), 81 (9), 45 (11).

HRMS $m / z$ : calcd. for $\mathrm{C}_{9} \mathrm{H}_{15} \mathrm{O}_{4} \mathrm{PS}: 250.0429$; found: 250.0424 . 


\section{Diethyl thiophen-2-ylmethyl phosphate $\mathbf{4 g}$}

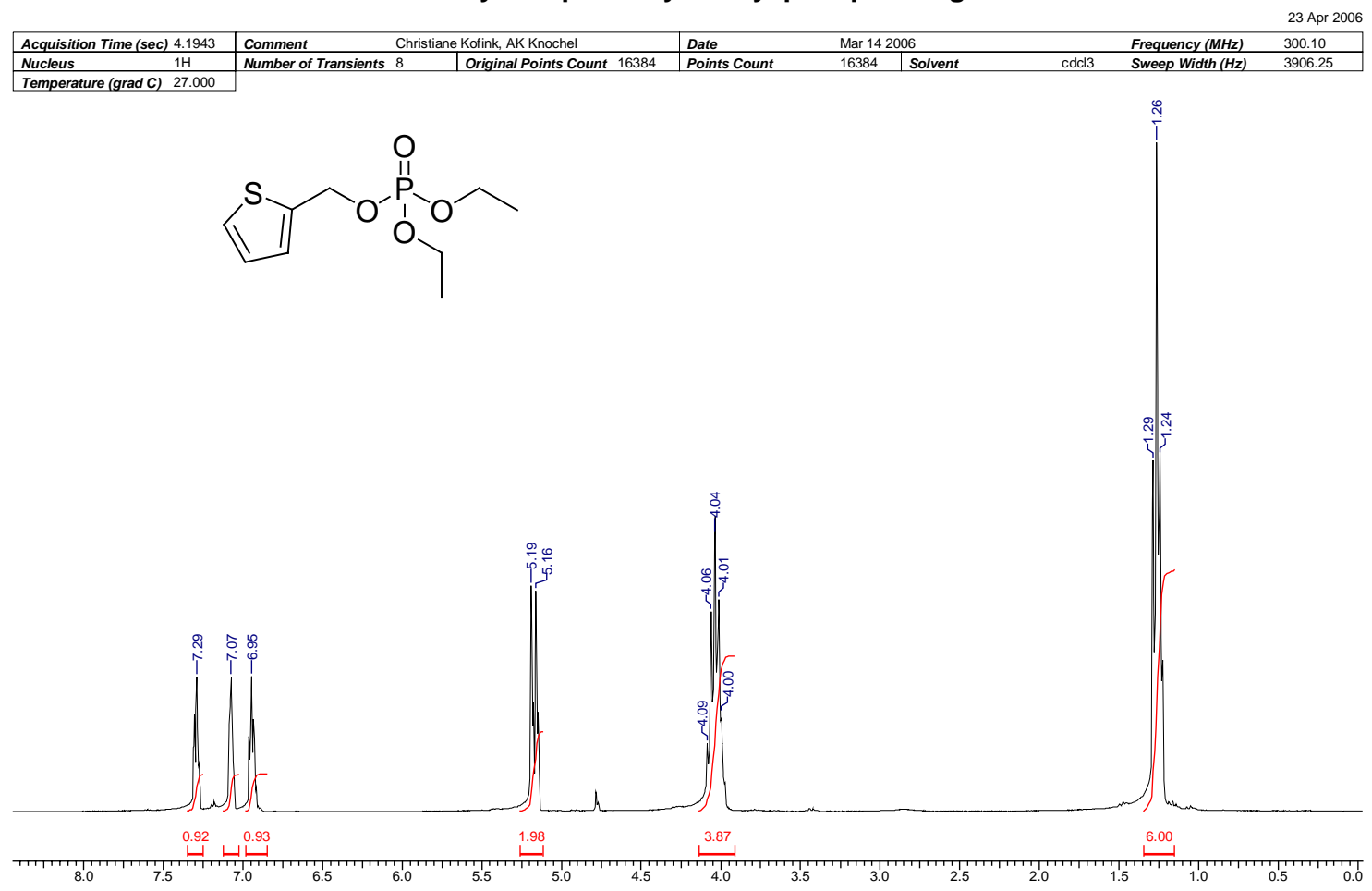

\section{Diethyl thiophen-2-ylmethyl phosphate $\mathbf{4 g}$}

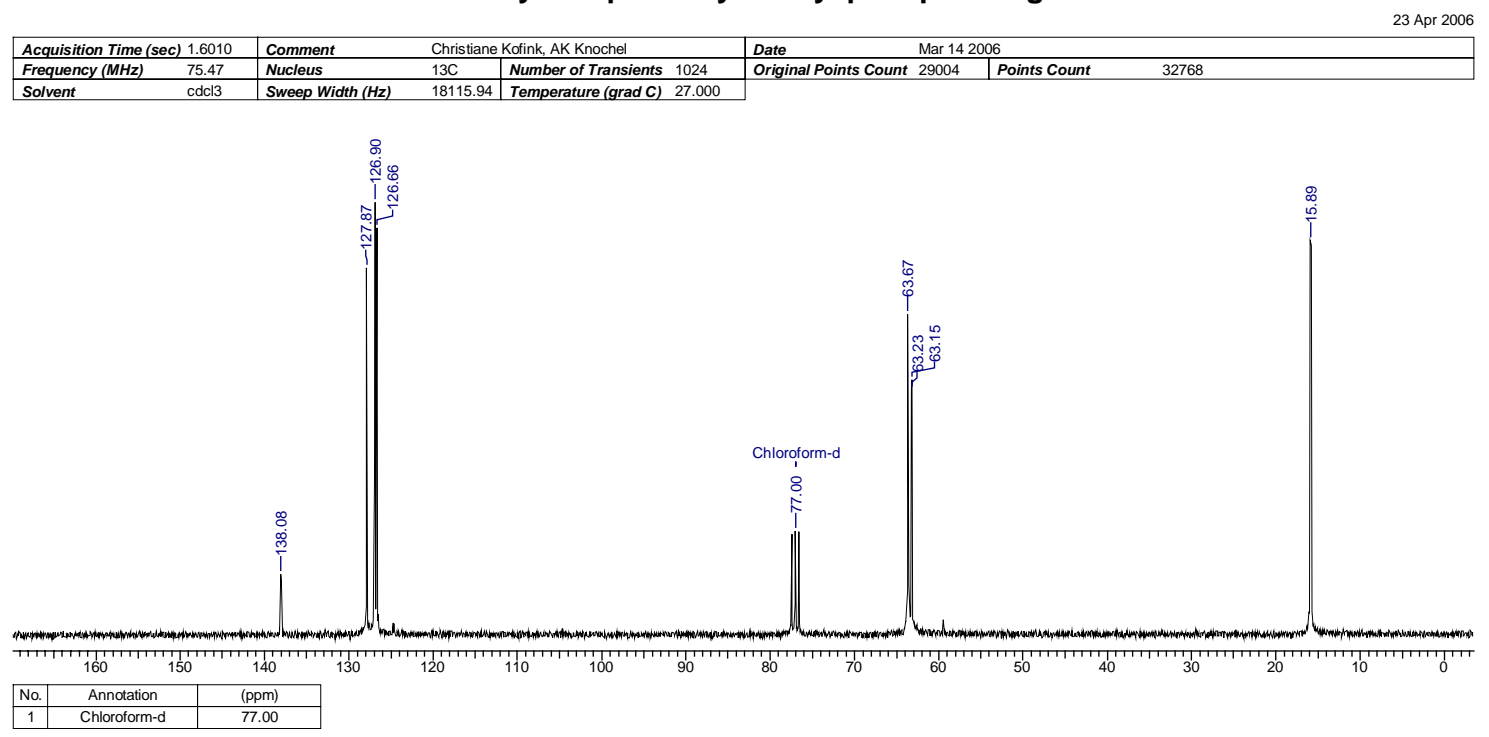




\section{Diethyl thiophen-3-ylmethyl phosphate 4h}

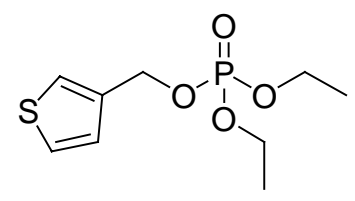

Prepared according to TP 3. Thiophen-3-ylmethanol (20.0 mmol, $2.88 \mathrm{~g}), \mathrm{NEt}_{3}$ (21.0 mmol, $2.95 \mathrm{~mL}$ ) and DMAP (2.00 mmol, $244 \mathrm{mg}$ ), are dissolved in THF. This mixture is treated with diethyl chlorophosphate $(21.0 \mathrm{mmol}, 3.62 \mathrm{~g})$ and stirred at $\mathrm{rt}$ for $8 \mathrm{~h}$. Standard work up and purification yielded $\mathbf{4 h}(2.29 \mathrm{~g}, 49 \%)$ as colourless liquid.

${ }^{1}$ H-NMR $\left(\mathrm{CDCl}_{3}, 300 \mathrm{MHz}, 25{ }^{\circ} \mathrm{C}\right): \delta=7.30-7.27(\mathrm{~m}, 2 \mathrm{H}), 7.11(\mathrm{~s}$ br, $1 \mathrm{H}), 5.05(\mathrm{~d}, J=$ $8.6 \mathrm{~Hz}, 2 \mathrm{H}), 4.09-3.99$ (m, 4H), 1.27 (t, $J=7.2 \mathrm{~Hz}, 6 \mathrm{H})$.

${ }^{13} \mathrm{C}-\mathrm{NMR}\left(\mathrm{CDCl}_{3}, 75 \mathrm{MHz}, 25{ }^{\circ} \mathrm{C}\right): \delta=136.98(\mathrm{~d}, J(\mathrm{C}, \mathrm{P})=6.6 \mathrm{~Hz}), 127.1,126.2,124.1$, $63.97(\mathrm{~d}, J(\mathrm{C}, \mathrm{P})=5.5 \mathrm{~Hz}), 63.59(\mathrm{~d}, J(\mathrm{C}, \mathrm{P})=5.5 \mathrm{~Hz}), 15.90(\mathrm{~d}, J(\mathrm{C}, \mathrm{P})=7.2 \mathrm{~Hz})$.

${ }^{31} \mathbf{P}-\mathbf{N M R}\left(\mathrm{CDCl}_{3}, 200 \mathrm{MHz}, 25^{\circ} \mathrm{C}\right): \delta=0.160$.

IR (KBr): 2983 (w), 1505 (w), 1442 (w), 1260 (s), 1162 (m), 1011 (vs), 967 (vs), 859 (m), $785(\mathrm{~s}), 750(\mathrm{~m}), 689(\mathrm{~m})$.

MS (70 eV, EI), m/z (\%): $250\left(66, \mathrm{M}^{+}\right), 221$ (28), 193 (36), 113 (76), 97 (100), 81 (11), 45 (16).

HRMS $m / z$ : calcd. for $\mathrm{C}_{9} \mathrm{H}_{15} \mathrm{O}_{4} \mathrm{PS}: 250.0429$; found: 250.0426 . 


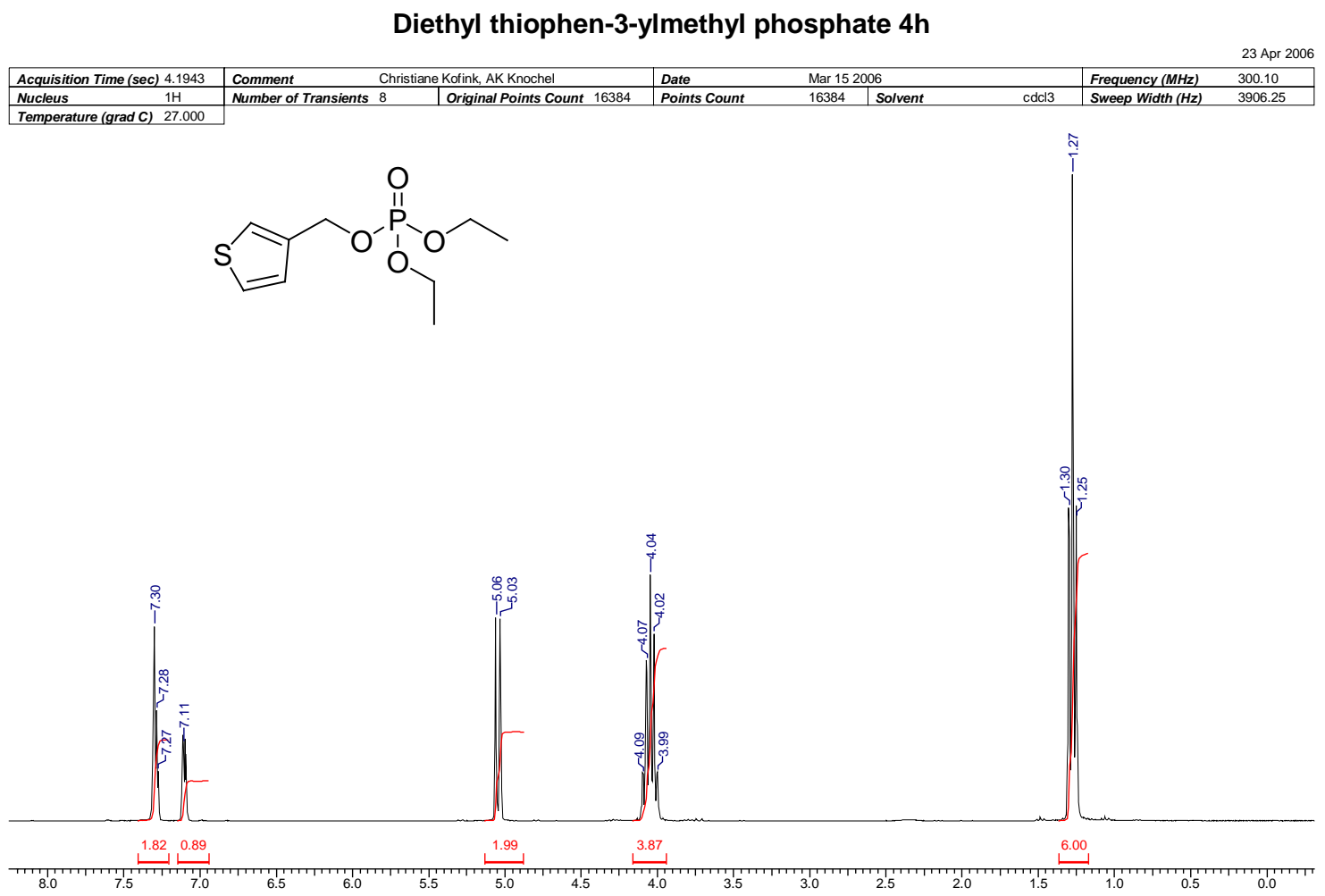

\section{Diethyl thiophen-3-ylmethyl phosphate $4 \mathrm{~h}$}

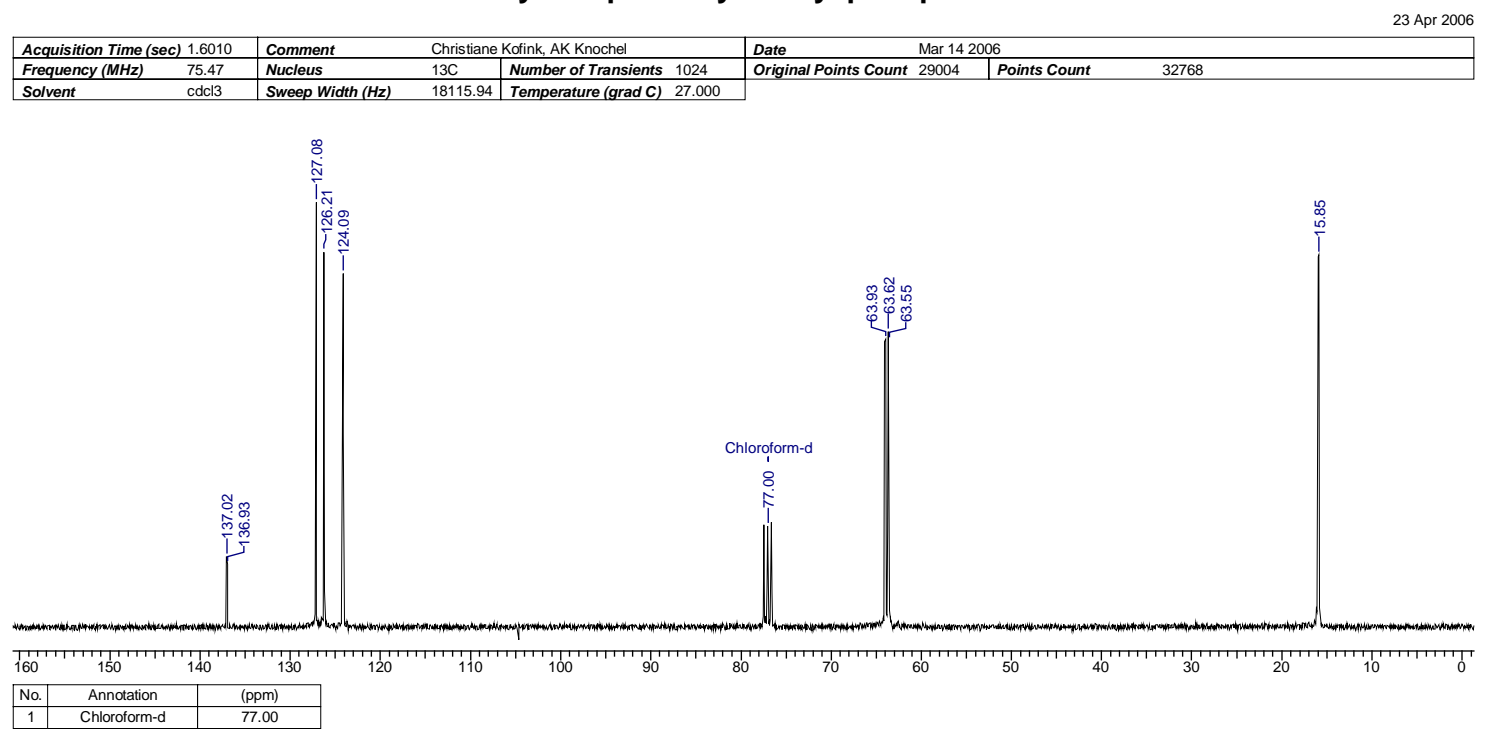




\section{2-Bromobenzyl diethyl phosphate 4i}

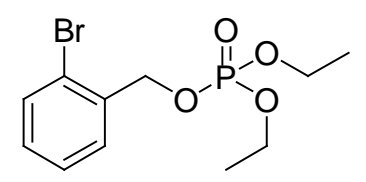

Prepared according to TP 3. (2-Bromophenyl)methanol (5.00 mmol, $935 \mathrm{mg}$ ), $\mathrm{NEt}_{3}$ $(5.5 \mathrm{mmol}, 0.77 \mathrm{~mL})$ and DMAP $(0.10 \mathrm{mmol}, 61 \mathrm{mg})$, are dissolved in THF. This mixture is treated with diethyl chlorophosphate $(5.50 \mathrm{mmol}, 949 \mathrm{mg})$ and stirred at $\mathrm{rt}$ for $4 \mathrm{~h}$. Standard work up and purification yielded $4 \mathbf{i}(1.26 \mathrm{~g}, 75 \%)$ as colourless liquid.

${ }^{1} \mathbf{H}-\mathbf{N M R}\left(\mathrm{CDCl}_{3}, 300 \mathrm{MHz}, 2{ }^{\circ} \mathrm{C}\right): \delta=7.49-7.43(\mathrm{~m}, 2 \mathrm{H})$, 7.29-7.24 (m, 1H), 7.14-7.09 (m, 1H), 5.07 (d, $J=7.2 \mathrm{~Hz}, 2 \mathrm{H}), 4.11-4.01(\mathrm{~m}, 4 \mathrm{H}), 1.25$ (t, $J=7.1 \mathrm{~Hz}, 6 \mathrm{H})$.

${ }^{13} \mathrm{C}-\mathrm{NMR}\left(\mathrm{CDCl}_{3}, 75 \mathrm{MHz}, 25{ }^{\circ} \mathrm{C}\right): \delta=135.38(\mathrm{~d}, J(\mathrm{C}, \mathrm{P})=7.9 \mathrm{~Hz}), 132.5,129.6,129.1$, 127.5, 122.3, $68.11(\mathrm{~d}, J(\mathrm{C}, \mathrm{P})=5.0 \mathrm{~Hz}), 63.85(\mathrm{~d}, J(\mathrm{C}, \mathrm{P})=5.9 \mathrm{~Hz}), 15.99(\mathrm{~d}, J(\mathrm{C}, \mathrm{P})=6.5 \mathrm{~Hz})$. ${ }^{31} \mathbf{P}-\mathbf{N M R}\left(\mathrm{CDCl}_{3}, 200 \mathrm{MHz}, 2{ }^{\circ} \mathrm{C}\right): \delta=-0.043$.

IR (KBr): 2983 (w), 1595 (w), 1442 (m), 1264 (s), 1164 (w), 1019 (vs), 976 (vs), 882 (m), $750(\mathrm{~m}), 659(\mathrm{~m})$.

MS (70 eV, EI), m/z (\%): 321 (51, M+), 293 (45), 265 (43), 185 (100), 171 (20), 125 (45), 89 (16), 63 (5).

HRMS $m / z$ : calcd. for $\mathrm{C}_{11} \mathrm{H}_{16} \mathrm{BrO}_{4} \mathrm{P}: 321.9970$; found: 321.9940 . 


\section{2-Bromobenzyl diethyl phosphate 4i}

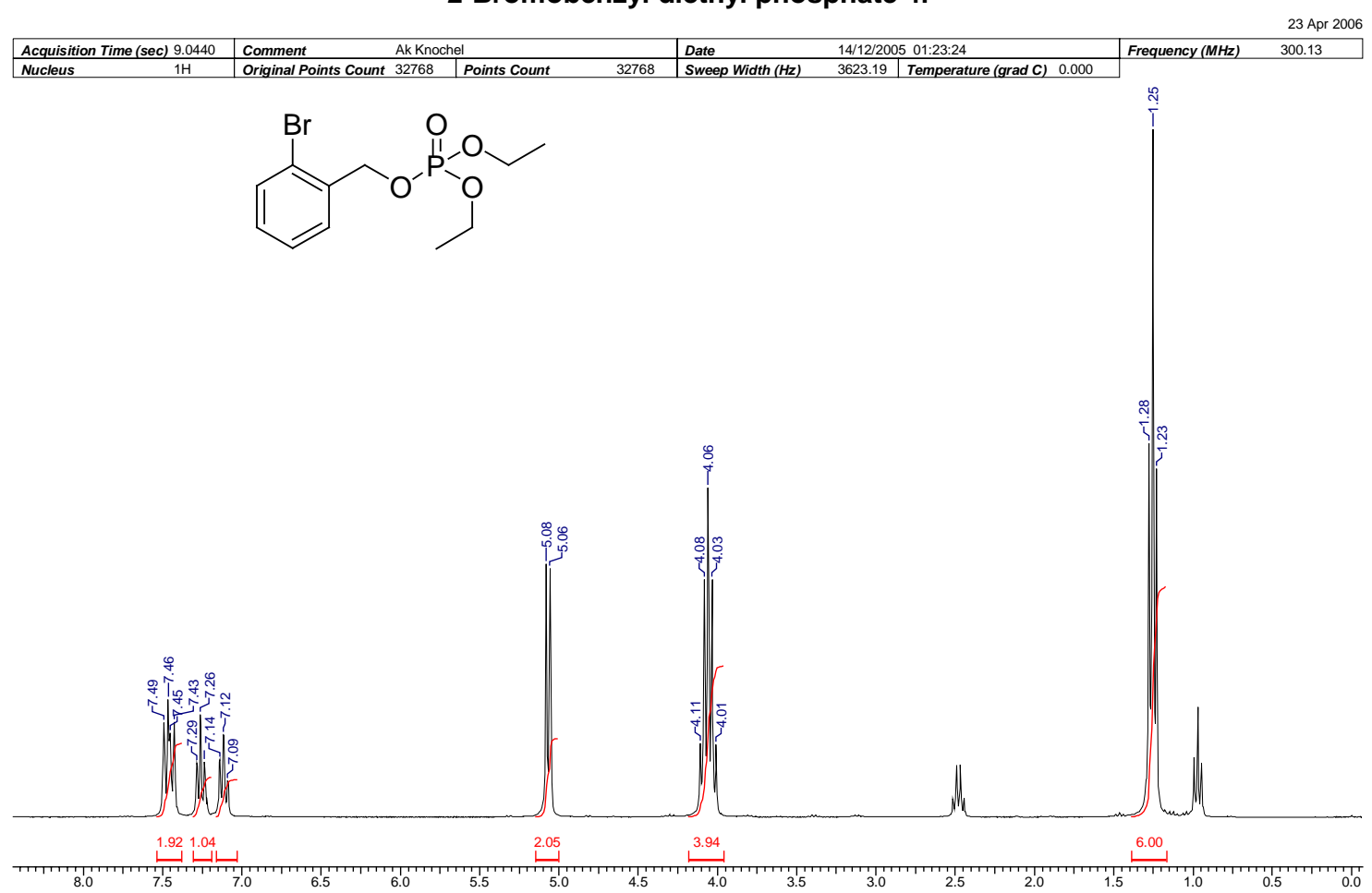

\section{2-Bromobenzyl diethyl phosphate 4i}

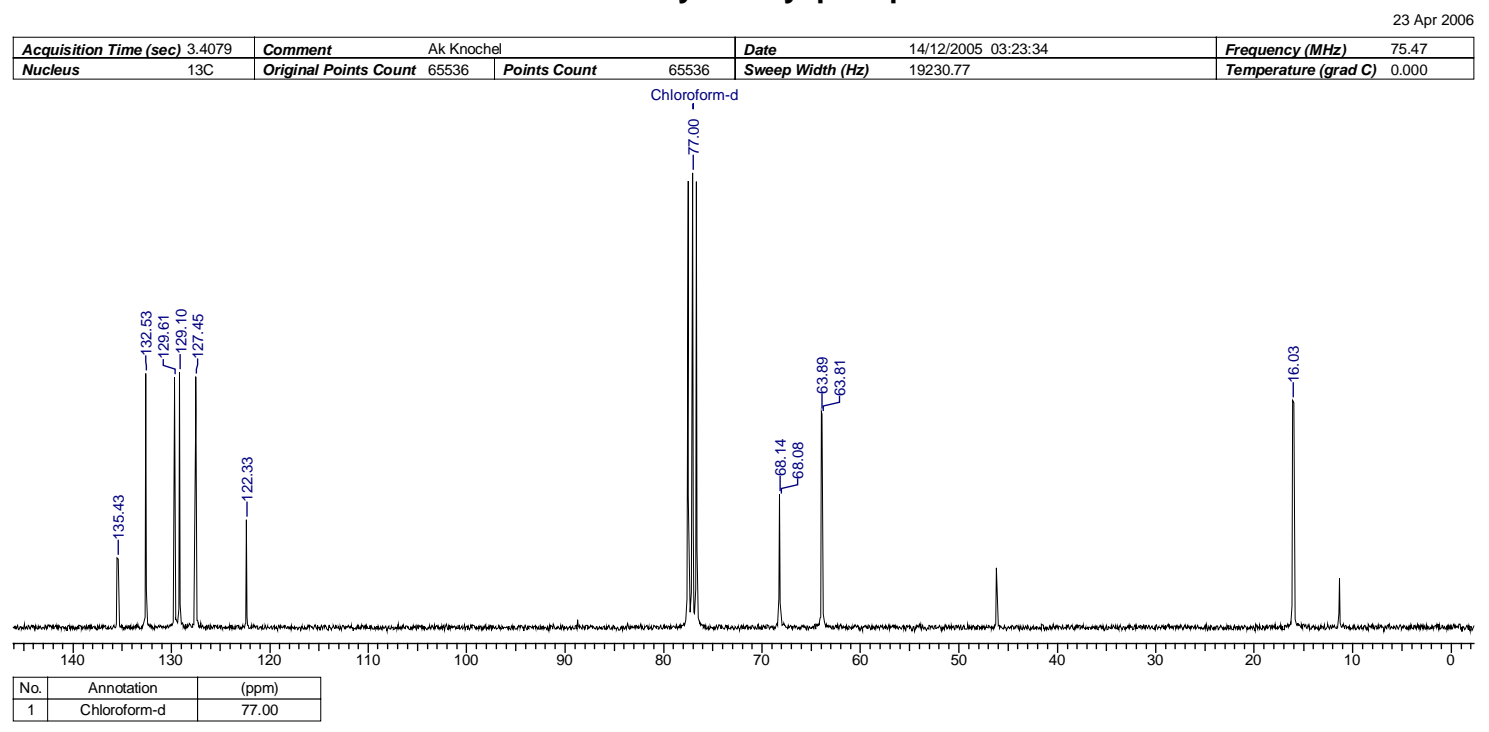




\section{Methyl 4-((diethoxyphosphoryloxy)methyl)benzoate 4j}

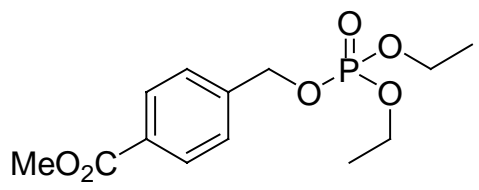

Prepared according to TP 3. Methyl 4-(hydroxymethyl)benzoate (9.50 mmol, $1.58 \mathrm{~g}), \mathrm{NEt}_{3}$ $(10.0 \mathrm{mmol}, 1.50 \mathrm{~mL})$ and DMAP $(1.00 \mathrm{mmol}, 122 \mathrm{mg})$, are dissolved in THF. This mixture is treated with diethyl chlorophosphate $(10.0 \mathrm{mmol}, 1.78 \mathrm{~g})$ and stirred at $\mathrm{rt}$ for $8 \mathrm{~h}$. Standard work up and purification yielded $\mathbf{4 j}(2.27 \mathrm{~g}, 79 \%)$ as colourless liquid.

${ }^{1} \mathbf{H}-\mathbf{N M R}\left(\mathrm{CDCl}_{3}, 300 \mathrm{MHz}, 25^{\circ} \mathrm{C}\right): \delta=7.89-7.87$ (m, 2H), 7.32-7.30 (m, 2H), 4.97-4.95 (m 2H), 3.96 (s br, 4H), 3.75 (s, 3H), 1.15 (s, 6H).

${ }^{13} \mathrm{C}-\mathrm{NMR}\left(\mathrm{CDCl}_{3}, 75 \mathrm{MHz}, 25{ }^{\circ} \mathrm{C}\right): \delta=166.2,140.77(\mathrm{~d}, J(\mathrm{C}, \mathrm{P})=6.7 \mathrm{~Hz}), 129.7,129.4$, $126.9,67.75(\mathrm{~d}, J(\mathrm{C}, \mathrm{P})=5.1 \mathrm{~Hz}), 63.57(\mathrm{~d}, J(\mathrm{C}, \mathrm{P})=5.6 \mathrm{~Hz}), 51.7,15.71(\mathrm{~d}, J(\mathrm{C}, \mathrm{P})=6.7 \mathrm{~Hz})$.

${ }^{31} \mathbf{P}$-NMR $\left(\mathrm{CDCl}_{3}, 200 \mathrm{MHz}, 25{ }^{\circ} \mathrm{C}\right): \delta=0.104$.

IR (KBr): 2985 (w), 1720 (s), 1616 (w), 1436 (m), 1272 (vs), 1178 (m), 1107 (m), 1010 (vs), 964 (vs), 872 (m), 841 (m), 754 (s), 700 (m).

MS (70 eV, EI), m/z (\%): $302\left(59, \mathrm{M}^{+}\right), 273$ (20 ), 245 (35), 231 (16), 165 (100 ), 149 (54), 125 (17), 89 (14).

HRMS $m / z$ : calcd. for $\mathrm{C}_{13} \mathrm{H}_{19} \mathrm{O}_{6} \mathrm{P}: 302.0919$; found: 302.0934 . 
Phosphate 4j

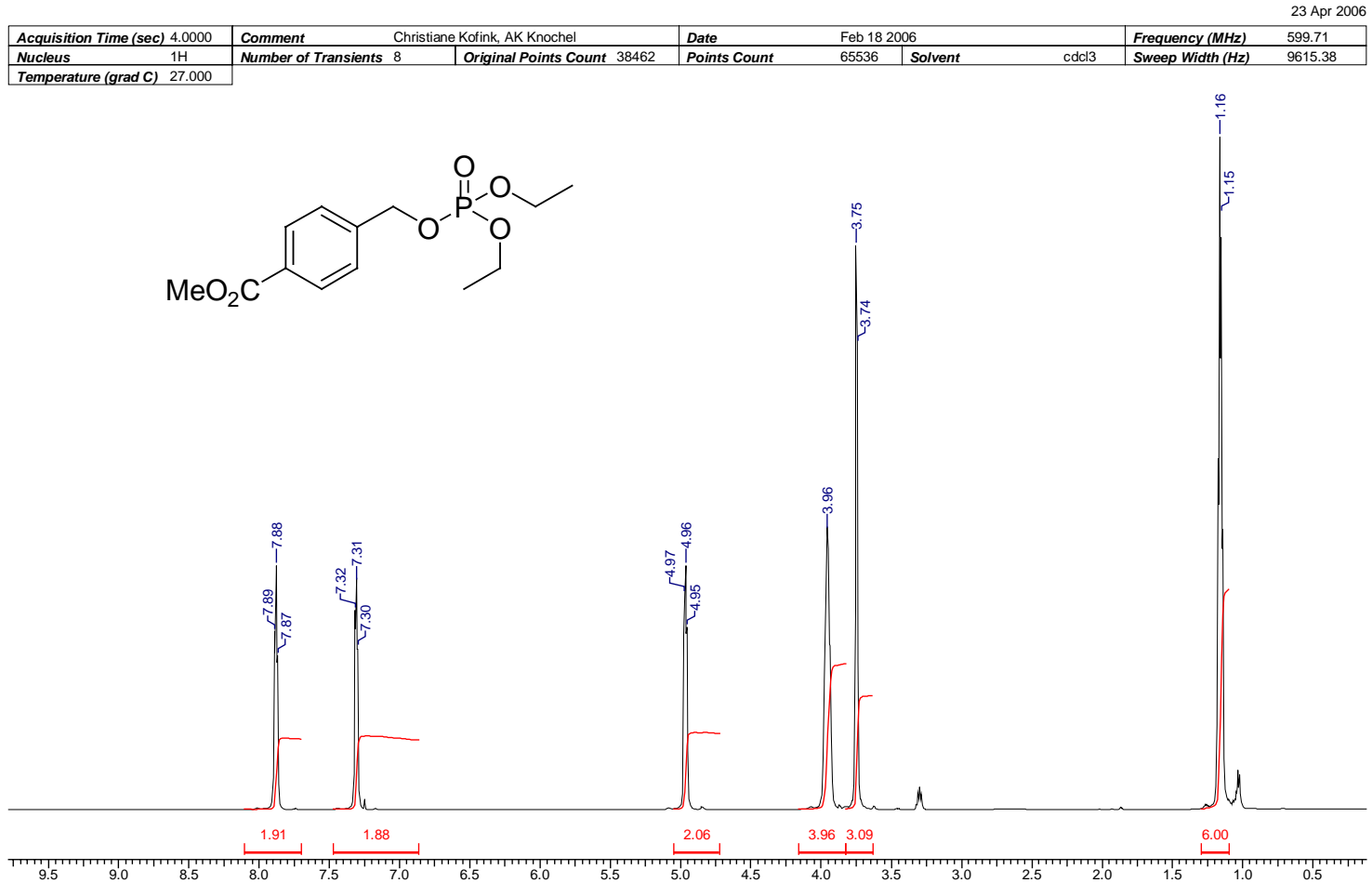

\section{Phosphate 4j}

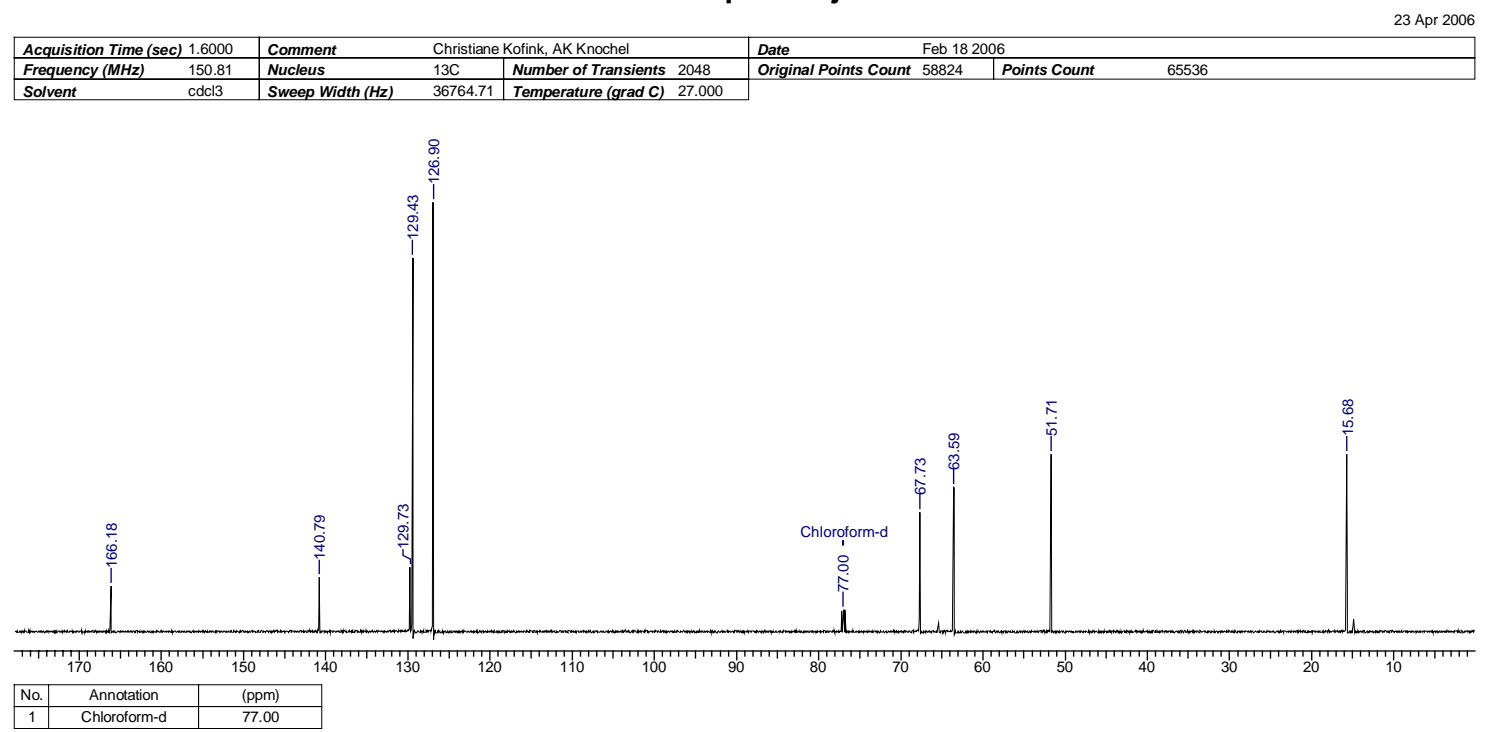




\section{Synthesis of Trimethoprim 1:}

\section{5-Bromo-2,4-di-tert-butoxypyrimidine 5}

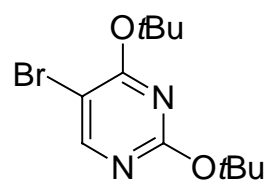

5-Bromo-2,4-dichloropyrimidine (10.0 mmol, $2.28 \mathrm{~g})$ is dissolved in dry THF (50 mL). Then a solution of sodium tert-butoxide $(25.0 \mathrm{mmol}, 2.40 \mathrm{~g})$ in THF $(100 \mathrm{~mL})$ is added dropwise at $\mathrm{rt}$ and the reaction mixture stirred for $5 \mathrm{~h}$. The reaction is quenched with water $(50 \mathrm{~mL})$ and extracted with EtOAc $(3 \times 50 \mathrm{~mL})$. Purification by flash chromatography yielded the desired product 5 as colorless solid $(2.79 \mathrm{~g}, 92 \%)$.

Mp.: $61^{\circ} \mathrm{C}$.

${ }^{1} \mathrm{H}-\mathrm{NMR}\left(\mathrm{CDCl}_{3}, 300 \mathrm{MHz}, 25^{\circ} \mathrm{C}\right): \delta=8.25(\mathrm{~s}, 1 \mathrm{H}), 1.65(\mathrm{~s}, 9 \mathrm{H}), 1.60(\mathrm{~s}, 9 \mathrm{H})$.

${ }^{13} \mathrm{C}-\mathrm{NMR}\left(\mathrm{CDCl}_{3}, 75 \mathrm{MHz}, 25{ }^{\circ} \mathrm{C}\right): \delta=165.6,162.9,158.9,99.5,83.2,80.7,28.3$.

IR (KBr): 2974 (w), 2930 (w), 1567 (s), 1538 (s), 1438 (m), 1389 (vs), 1361 (vs), 1287 (m), 1228 (m), 1151 (vs), 1088 (vs), 1014 (s), 938 (m), 900 (s), 846 (s), 782 (s), 750 (m), 681 (m). MS (70 eV, EI), m/z (\%):302 (2100, M+), 246 (8), 191 (100), 147 (16), 57 (54).

HRMS $m / z$ : calcd. for $\mathrm{C}_{12} \mathrm{H}_{19} \mathrm{BrN}_{2} \mathrm{O}_{2}: 302.0630$; found: 302.0643 . 


\section{5-Bromo-2,4-di-tert-butoxypyrimidine 5}

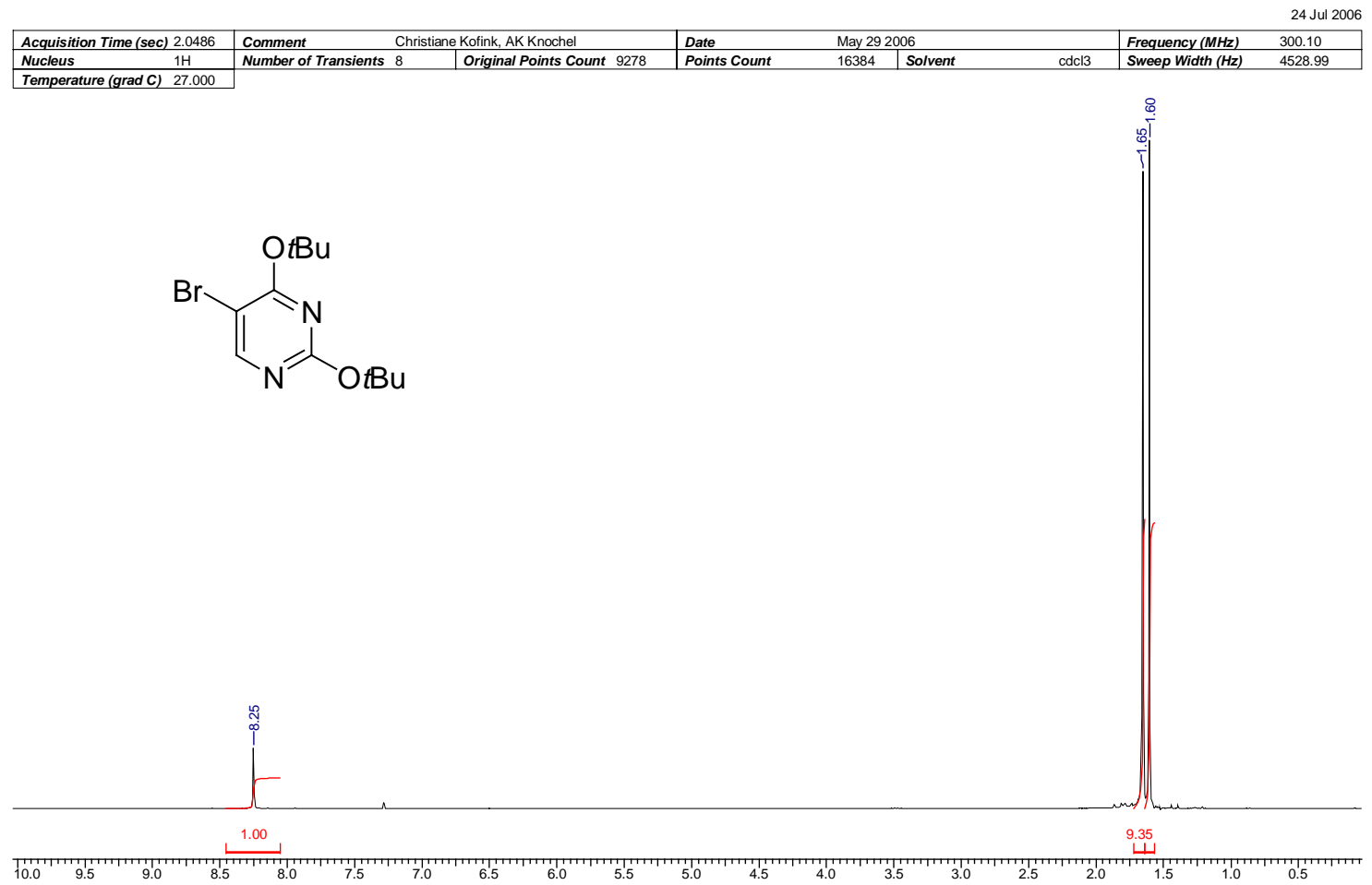

\section{5-Bromo-2,4-di-tert-butoxypyrimidine 5}

\begin{tabular}{|ll|ll|l|l|l|l|}
\hline Acquisition Time (sec) & 1.6010 & Comment & Christiane Kofink, AK Knochel & Date & \multicolumn{2}{c|}{ May 292006} \\
\hline Frequency $($ MHz) & 75.47 & Nucleus & 13C & Number of Transients 2048 & Original Points Count 29004 & Points Count \\
\hline
\end{tabular}

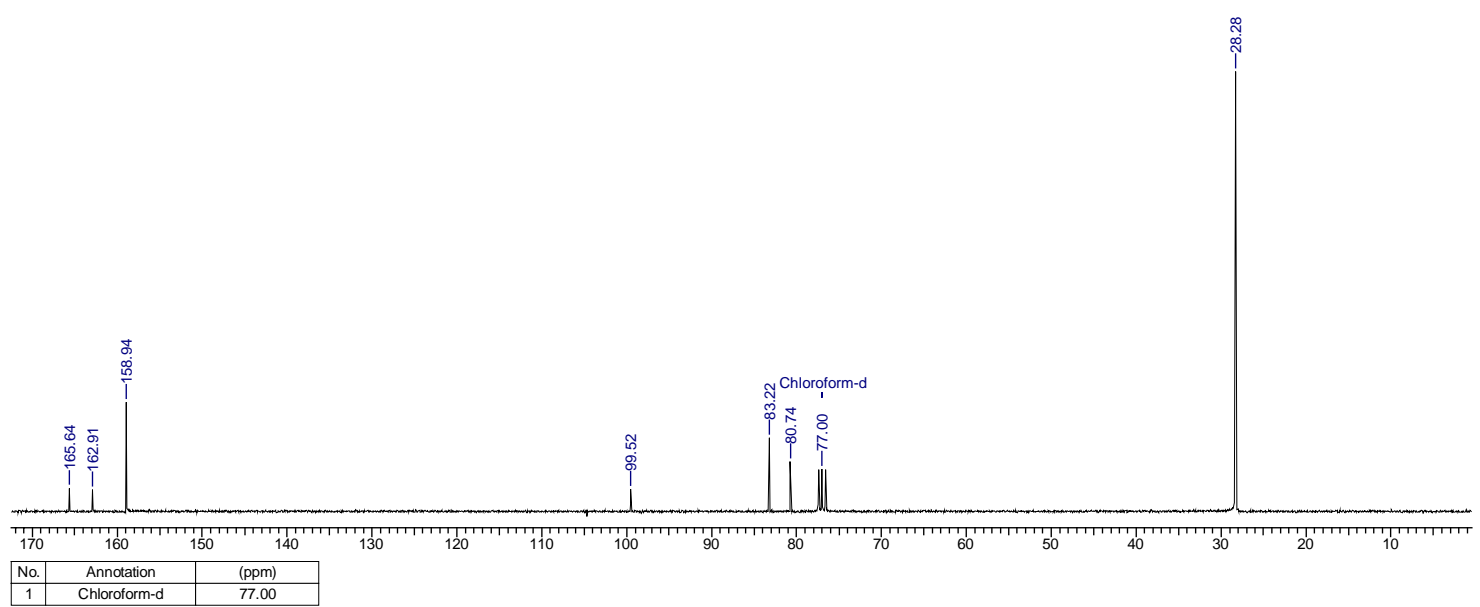




\section{Diethyl 3,4,5-trimethoxybenzyl phosphate 6}

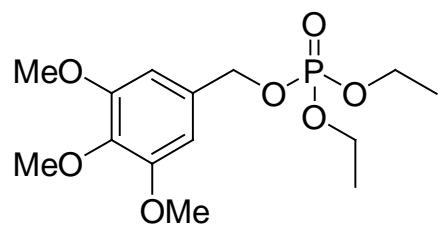

Prepared according to TP 3. (3,4,5-Trimethoxyphenyl)methanol (15.0 mmol, $2.97 \mathrm{~g}), \mathrm{NEt}_{3}$ (16.0 mmol, $2.24 \mathrm{~mL})$ and DMAP (1.50 mmol, $183 \mathrm{mg})$, are dissolved in THF. This mixture is treated with diethyl chlorophosphate $(16.0 \mathrm{mmol}, 2.76 \mathrm{~g})$ and stirred at $\mathrm{rt}$ for $4 \mathrm{~h}$. Standard work up and purification yielded $6(4.17 \mathrm{~g}, 83 \%)$ as colourless liquid.

${ }^{1} \mathbf{H}-\mathbf{N M R}\left(\mathrm{CDCl}_{3}, 300 \mathrm{MHz}, 25{ }^{\circ} \mathrm{C}\right): \delta=6.58-6.55(\mathrm{~m}, 2 \mathrm{H}), 4.96-4.91(\mathrm{~m}, 2 \mathrm{H}), 4.07-4.01(\mathrm{~m}$, $4 \mathrm{H}), 3.82-3.76(\mathrm{~m}, 9 \mathrm{H}), 1.27-1.24(\mathrm{~m}, 6 \mathrm{H})$.

${ }^{13} \mathrm{C}-\mathrm{NMR}\left(\mathrm{CDCl}_{3}, 75 \mathrm{MHz}, 25{ }^{\circ} \mathrm{C}\right): \delta=153.1,137.8,131.45(\mathrm{~d}, J(\mathrm{C}, \mathrm{P})=6.6 \mathrm{~Hz}), 104.8$, $69.05(\mathrm{~d}, J(\mathrm{C}, \mathrm{P})=5.5 \mathrm{~Hz}), 63.63(\mathrm{~d}, J(\mathrm{C}, \mathrm{P})=5.9 \mathrm{~Hz}), 60.6,55.9,15.92(\mathrm{~d}, J(\mathrm{C}, \mathrm{P})=6.6 \mathrm{~Hz})$.

${ }^{31} \mathbf{P}-\mathbf{N M R}\left(\mathrm{CDCl}_{3}, 200 \mathrm{MHz}, 25^{\circ} \mathrm{C}\right): \delta=0.230$.

IR (KBr): 2983 (w), 1593 (m), 1508 (m), 1462 (m), 1334 (m), 1261 (s), 1249 (s), 1227 (vs), 1022 (vs), 969 (vs), 822 (w).

MS (70 eV, EI), m/z (\%):334 (100, M+), 305 (17), 277 (8), 181 (69), 165 (40), 137 (5).

HRMS $m / z$ : calcd. for $\mathrm{C}_{14} \mathrm{H}_{23} \mathrm{O}_{7} \mathrm{P}: 334.1181$; found: 334.1164 . 
Diethyl 3,4,5-trimethoxybenzyl phosphate 6

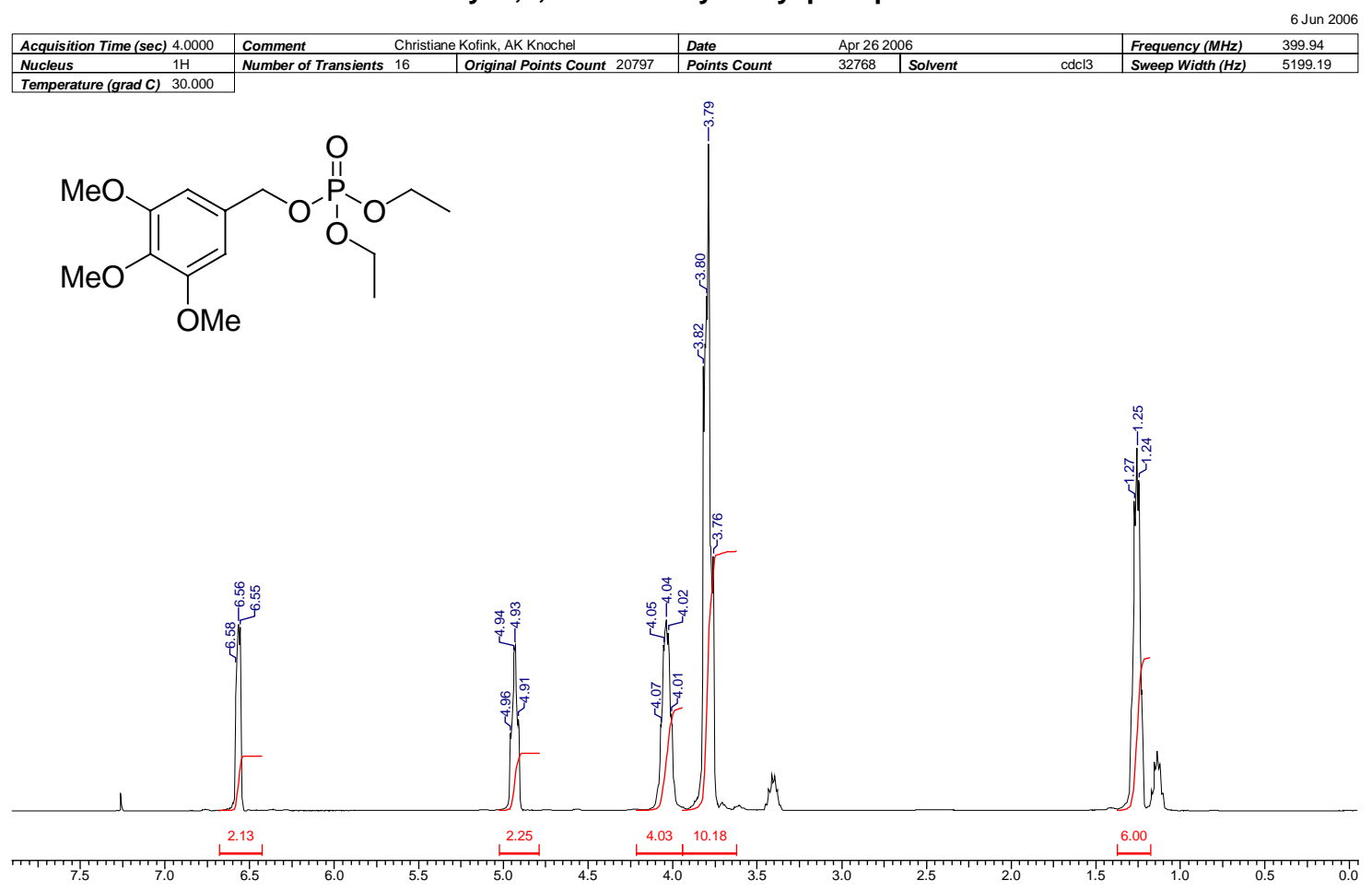

Diethyl 3,4,5-trimethoxybenzyl phosphate 6
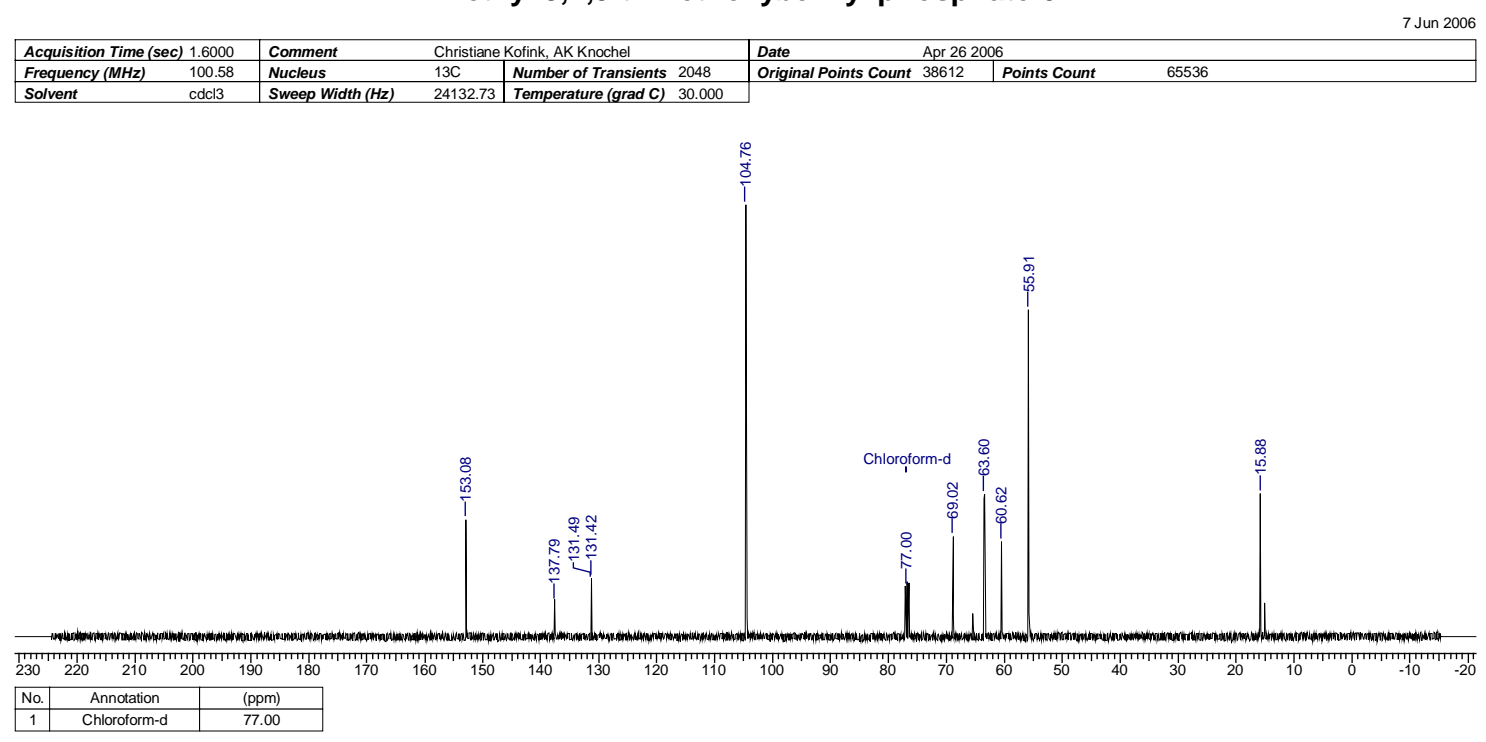


\section{2,4-Di-tert-butoxy-5-(3,4,5-trimethoxybenzyl)pyrimidine 7}

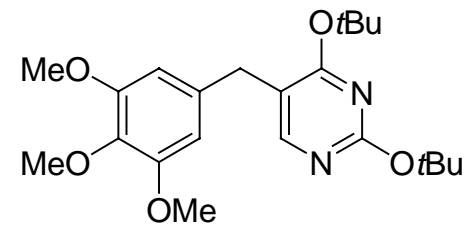

Prepared according to the TP 2. To a solution of 5-bromo-2,4-di-tert-butoxypyrimidine $(2.20 \mathrm{~g}, 7.25 \mathrm{mmol})$ in $5.0 \mathrm{~mL} \mathrm{DME}, i \mathrm{PrMgCl} \cdot \mathrm{LiCl}(5.95 \mathrm{~mL}, 7.73 \mathrm{mmol}, 1.3 \mathrm{M}$ in THF) is added dropwise via syringe at $0{ }^{\circ} \mathrm{C}$. The solution is stirred for $4 \mathrm{~h}$, than $\mathrm{CuBr}(69 \mathrm{mg}$, $10 \mathrm{~mol} \%)$ and $\mathrm{P}(\mathrm{OEt})_{3}(160 \mathrm{mg}, 20 \mathrm{~mol} \%)$ is added. This mixture is added over $30 \mathrm{~min}$ via canula to a prewarmed mixture of diethyl 3,4,5-trimethoxybenzyl phosphate $(1.62 \mathrm{~g}, 4.83$ mmol) and TBAI (177 mg, $10 \mathrm{~mol} \%)$. The reaction mixture is heated at $60{ }^{\circ} \mathrm{C}$ for $1 \mathrm{~h}$. The usual workup and purification by flash chromatography (pentane/ether 1:1) yielded 7 as colourless solid $(1.56 \mathrm{~g}, 81 \%)$.

Mp:. $78^{\circ} \mathrm{C}$.

${ }^{1} \mathrm{H}-\mathrm{NMR}\left(\mathrm{CDCl}_{3}, 600 \mathrm{MHz}, 25^{\circ} \mathrm{C}\right): \delta=7.97(\mathrm{~s}, 1 \mathrm{H}), 6.40(\mathrm{~s}, 2 \mathrm{H}), 3.81(\mathrm{~s}, 9 \mathrm{H}), 3.64(\mathrm{~s}, 2 \mathrm{H})$, $1.60(\mathrm{~s}, 9 \mathrm{H}), 1.56(\mathrm{~s}, 9 \mathrm{H})$.

${ }^{13} \mathrm{C}-\mathrm{NMR}\left(\mathrm{CDCl}_{3}, 75 \mathrm{MHz}, 25{ }^{\circ} \mathrm{C}\right): \delta=168.1,163.0,157.4,153.1,135.7,115.2,105.7,81.3$, $80.0,60.9,56.1,33.7,28.4$.

IR (KBr): 2975 (w), 2932 (w), 1591 (s), 1554 (s), 1506 (m), 1456 (m), 1405 (vs), 1363 (s), 1332 (m), 1236 (m), 1150 (s), 1123 (vs), 1053 (s), 1011 (s), 936 (m), 805 (m).

MS (70 eV , EI), m/z (\%): 404 (36, M+ $), 348$ (26), 292 (100), 261 (46), 41 (14).

HRMS $m / z$ : calcd. for $\mathrm{C}_{22} \mathrm{H}_{32} \mathrm{~N}_{2} \mathrm{O}_{5}$ : 404.2311; found: 404.2321 . 


\section{Diarylmethane 7}

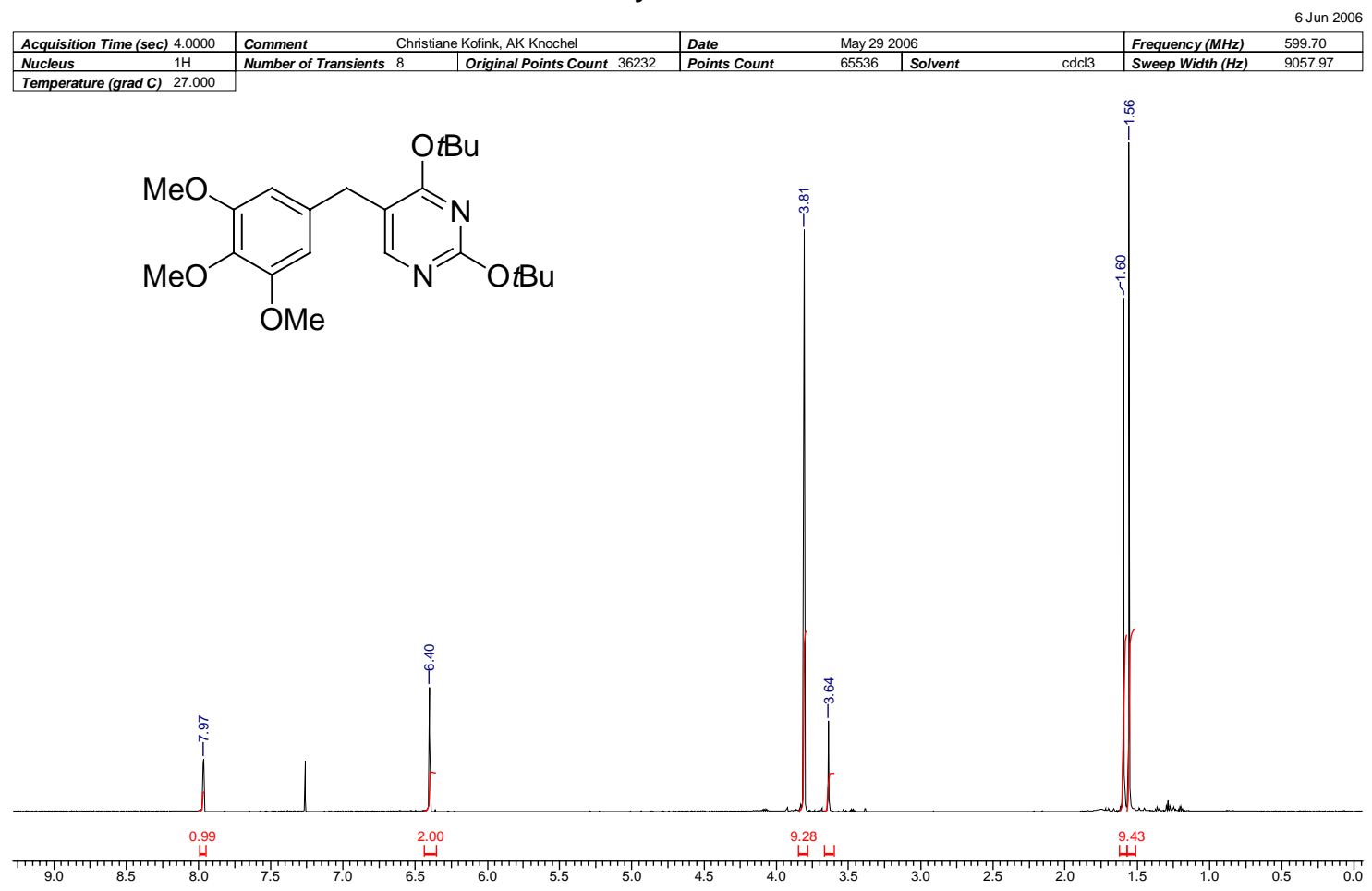

Diarylmethane 7
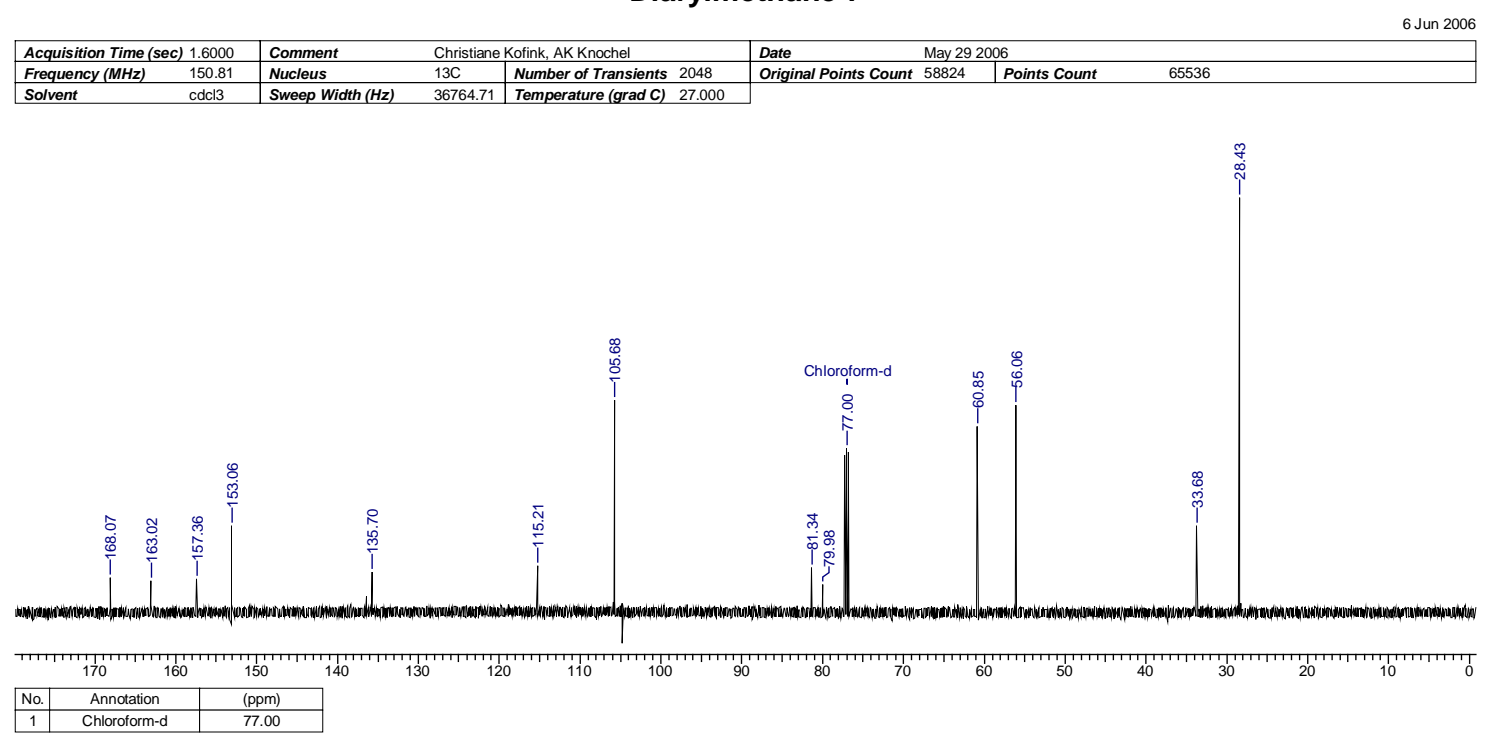


\section{5-(3,4,5-Trimethoxybenzyl)pyrimidine-2,4(1H,3H)-dione}

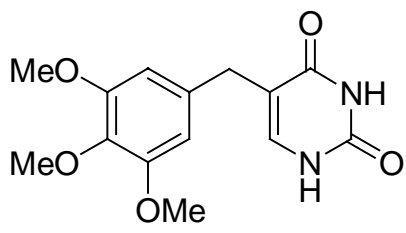

2,4-Di-tert-butoxy-5-(3,4,5-trimethoxybenzyl)pyrimidine 6 was dissolved in $\mathrm{MeOH}(5.0 \mathrm{~mL})$. Than conc. $\mathrm{HCl}(2.0 \mathrm{~mL})$ was added dropwise and immediately a precipitate was formed. The reaction mixture was stirred $15 \mathrm{~min}$ at $\mathrm{rt}$, than the precipitate was filtered off and washed with cold $\mathrm{MeOH}$ and dried in vacuo to yield the desired product as colourless solid (87\%, $1.04 \mathrm{~g})$. The compound was used for the next step to prepare 2,4-Dichloro-5-(3,4,5trimethoxybenzyl)pyrimidine 7 without further pruification.

Mp:. $216^{\circ} \mathrm{C}$.

${ }^{1}$ H-NMR (DMSO- $d^{6}, 400 \mathrm{MHz}, 25{ }^{\circ} \mathrm{C}$ ): $\delta=11.01$ (s br, 1H), 11.63 (s br, 1H), 7.17 (d, $J=$ $5.9 \mathrm{~Hz}, 1 \mathrm{H}), 6.51(\mathrm{~s}, 2 \mathrm{H}), 3.69$ (s, 6H), 3.58 (s, 3H), 3.39 (s, 2H).

${ }^{13}$ C-NMR (DMSO- $d^{6}, 100 \mathrm{MHz}, 25{ }^{\circ} \mathrm{C}$ ): $\delta=164.3,152.7,151.3,138.7,135.8,135.6,111.8$, $105.9,59.9,55.8,31.9$.

IR (KBr): 3130 (w), 1712 (m), 1667 (vs), 1585 (s), 1508 (m), 1422 (vs), 1241 (s), 1224 (s); 1129 (vs), 993 (m), 844 (m), 761 (m), $686(\mathrm{~m})$.

MS (70 eV, EI), m/z (\%): 292 (100, M+), 261 (32), 234 (8), 191 (6), 146 (4), 77 (3).

HRMS $m / z$ : calcd. for $\mathrm{C}_{14} \mathrm{H}_{16} \mathrm{~N}_{2} \mathrm{O}_{5}$ : : 292.1059; found: 292.1048 . 


\section{Uracilderivative to compound 8}

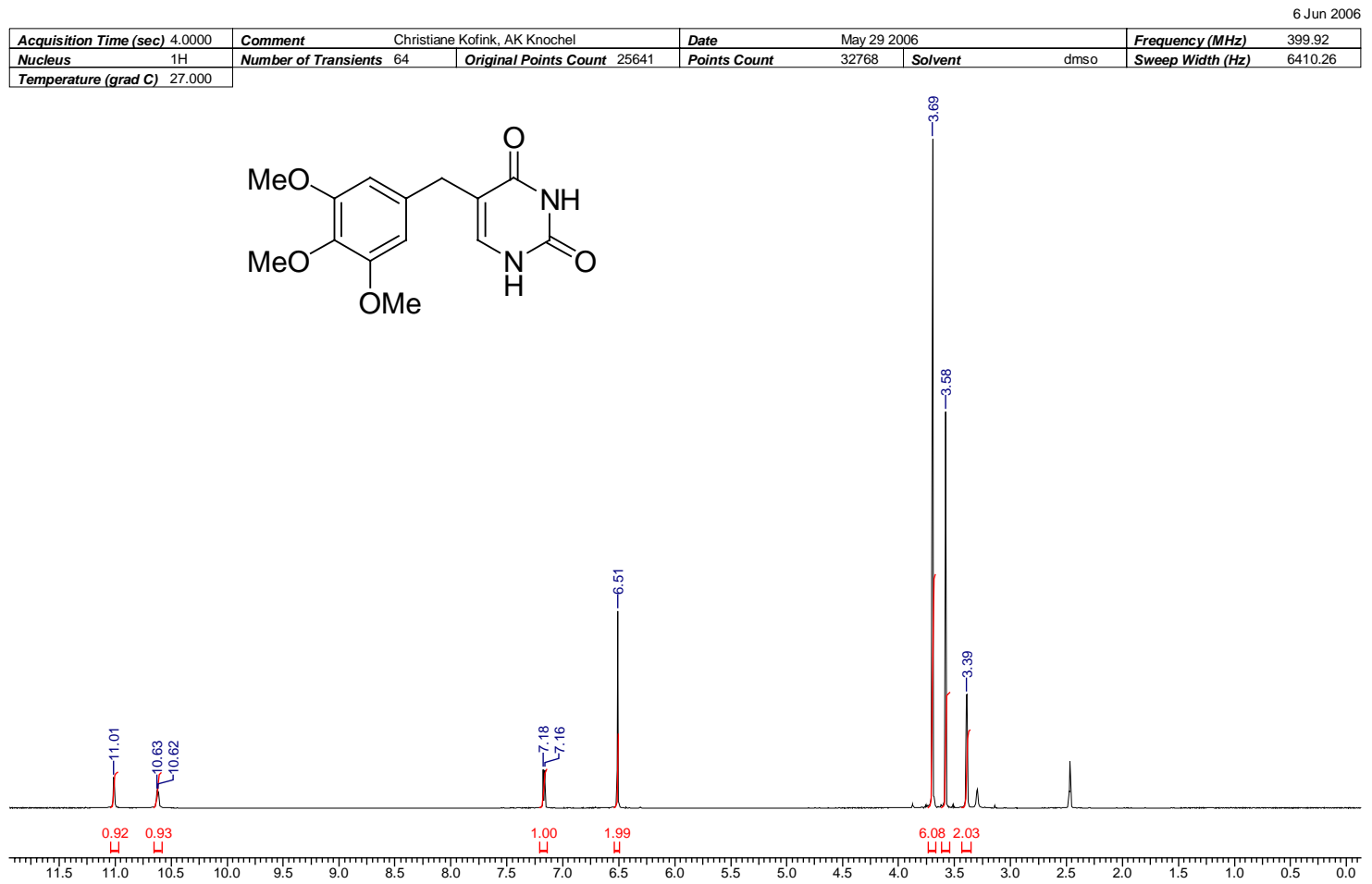

\section{Uracil derivative to compound 8}
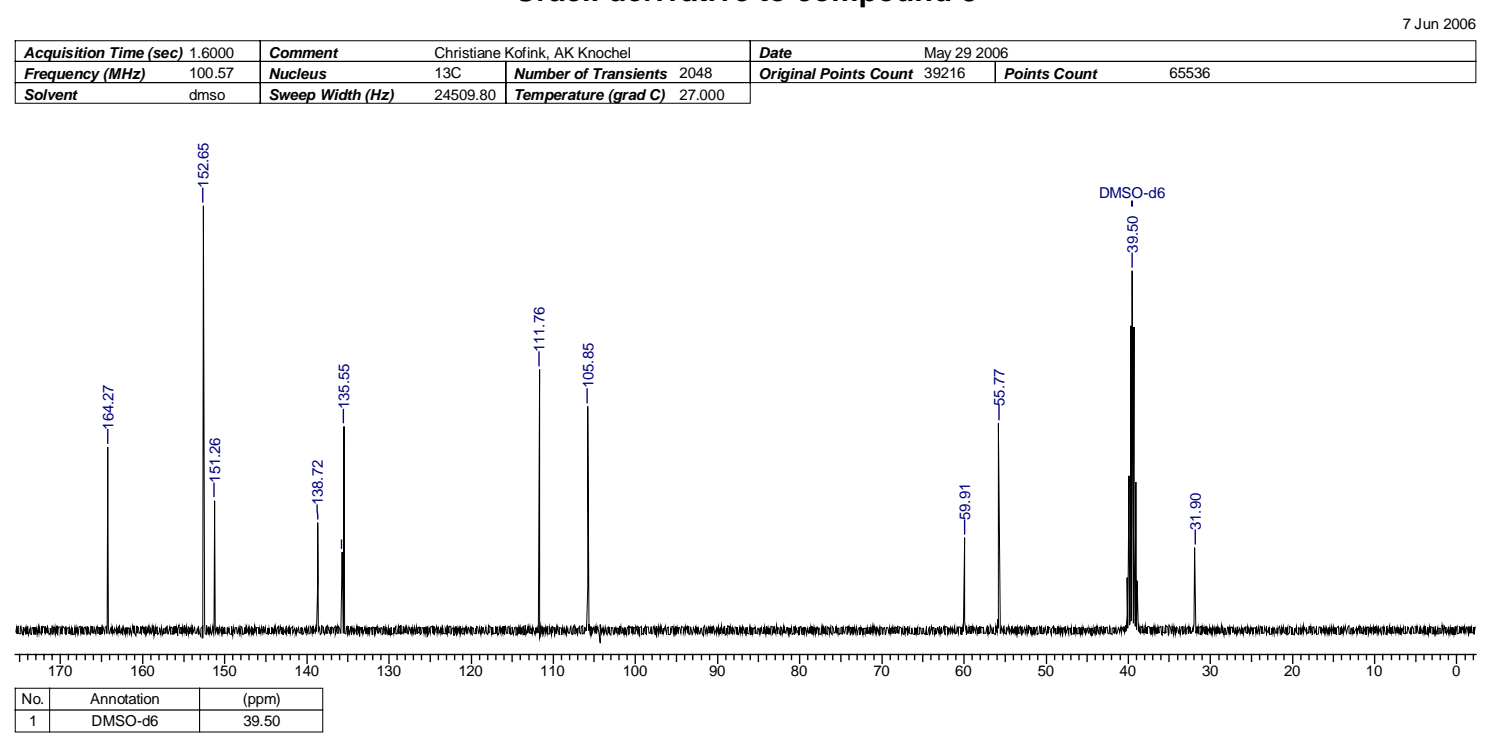


\section{2,4-Dichloro-5-(3,4,5-trimethoxybenzyl)pyrimidine 8}

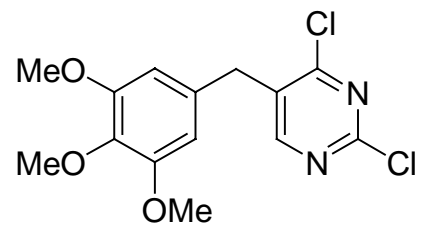

To a stirred mixture of $\mathrm{POCl}_{3}(1.02 \mathrm{~g}, 8.75 \mathrm{mmol})$ and $N, N$-dimethylbenzenamine $(640 \mathrm{mg}$, $5.25 \mathrm{mmol})$ in a $25 \mathrm{~mL}$ schlenk flask with reflux condenser the 5-(3,4,5trimethoxybenzyl)pyrimidine-2,4(1H,3H)-dione was added portion wise over $30 \mathrm{~min}$. Than the reaction mixture was refluxed for $1 \mathrm{~h}$ at $110{ }^{\circ} \mathrm{C}$. Afterwards the reaction mixture was pured on ice, extracted with diethyl ether, and the organic layer dried over $\mathrm{Na}_{2} \mathrm{SO}_{4}$. Purification by flash chromatography (ether) yielded $\mathbf{8}$ as colourless solid (990 mg, 86\%).

Mp:. $131^{\circ} \mathrm{C}$.

${ }^{1} \mathbf{H}-\mathrm{NMR}\left(\mathrm{CDCl}_{3}, 600 \mathrm{MHz}, 25^{\circ} \mathrm{C}\right): \delta=8.27(\mathrm{~s}, 1 \mathrm{H}), 6.37(\mathrm{~s}, 2 \mathrm{H}), 3.96(\mathrm{~s}, 2 \mathrm{H}), 3.82(\mathrm{~s}, 3 \mathrm{H})$, $3.81(\mathrm{~s}, 6 \mathrm{H})$.

${ }^{13} \mathrm{C}-\mathrm{NMR}\left(\mathrm{CDCl}_{3}, 150 \mathrm{MHz}, 25{ }^{\circ} \mathrm{C}\right): \delta=162.0,160.2,158.4,153.6,137.3,131.8,131.5$, $105.9,60.8,56.1,35.8$.

IR (KBr): 2961 (w), 2940 (w), 2840 (w), 1593 (w), 15008 (m), 1456 (m), 1421 (m), 1388 (m), 1338 (m), 1293 (w), 1251 (m), 1175 (m), 1125 (vs), 1080 (m), 1005 (s), 855 (s), 836 (s), $737(\mathrm{~m}), 686(\mathrm{~m})$.

MS (70 eV, EI), m/z (\%): 328 (100, M+), 313 (66), 249 (6), 234 (15), 161 (9), 124 (6).

HRMS $m / z$ : calcd. for $\mathrm{C}_{14} \mathrm{H}_{14} \mathrm{Cl}_{2} \mathrm{~N}_{2} \mathrm{O}_{3}: 328.0381$; found: 328.0383 . 


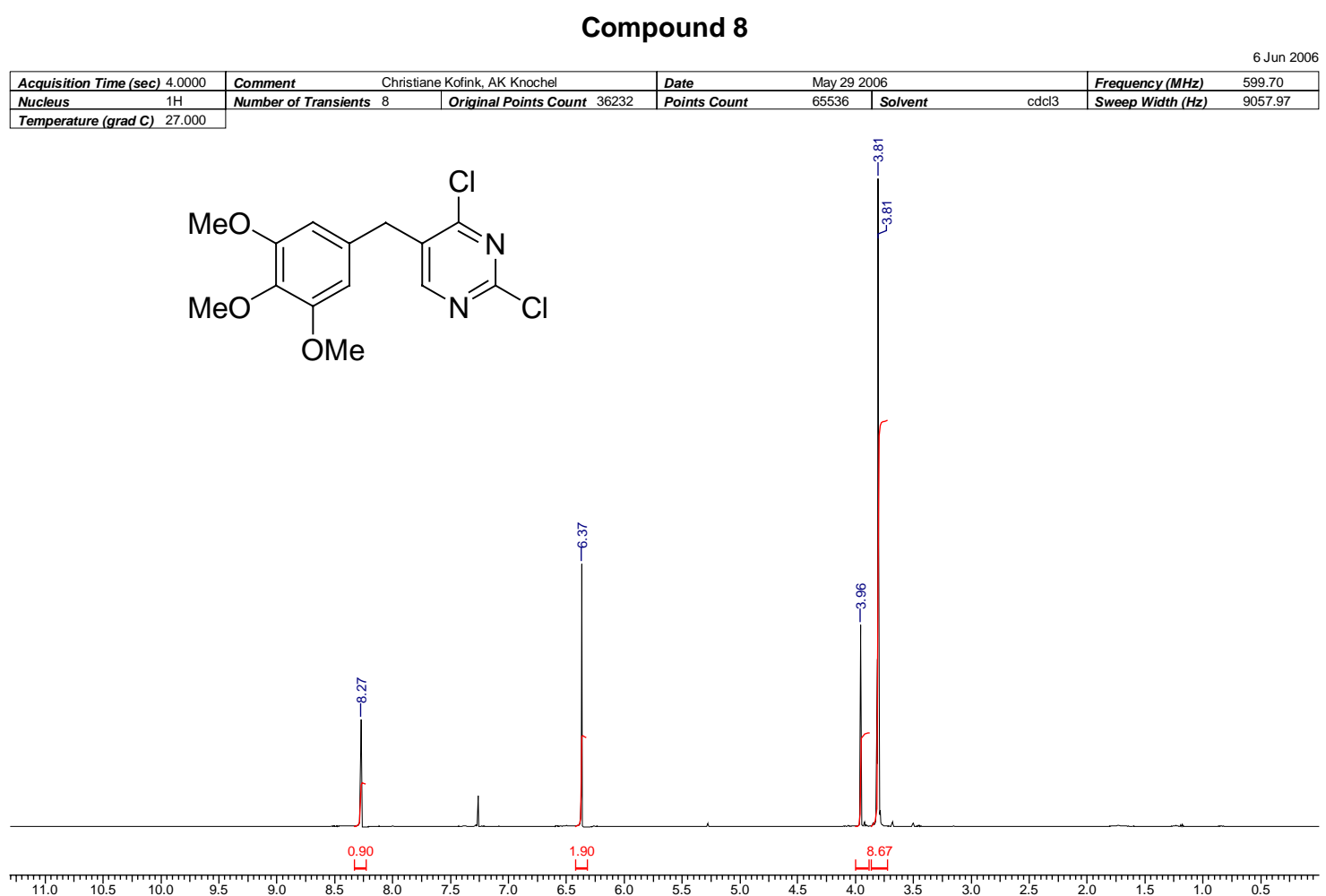

\section{Compound 8}
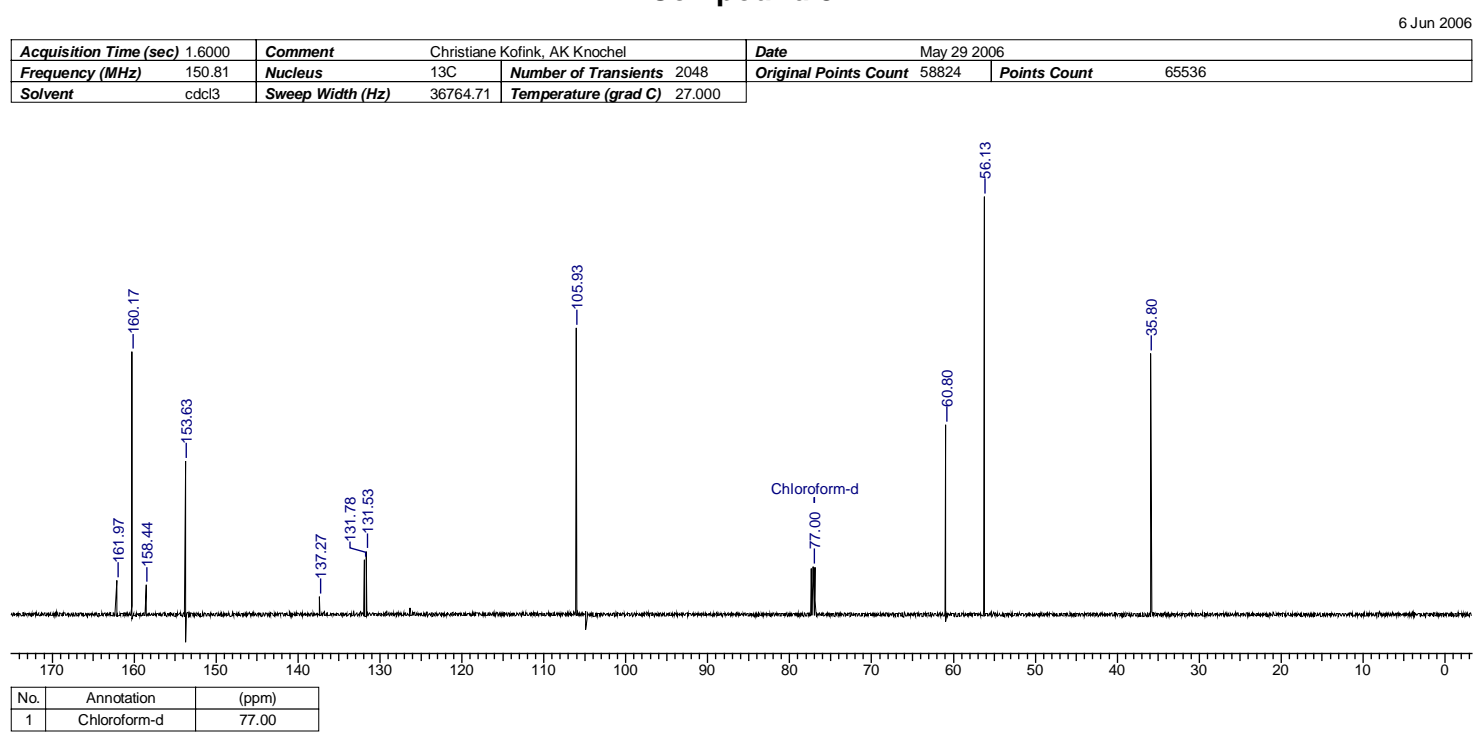


\section{5-(3,4,5-Trimethoxybenzyl)pyrimidine-2,4-diamine 1 (Trimethoprim)}

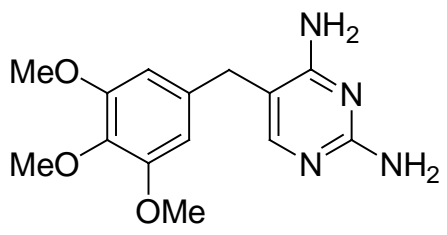

2,4-Dichloro-5-(3,4,5-trimethoxybenzyl)pyrimidine 7 was suspensioned in a solution of $\mathrm{NH}_{3}$ in $\mathrm{MeOH}(10 \mathrm{~mL}, 7 \mathrm{~N})$ in an autoclave and heated in the oven at $175^{\circ} \mathrm{C}$ for $6 \mathrm{~h}$. Than the solvent is removed in vacuo. Recrystallization in $\mathrm{MeOH}$, yielded the desired product $\mathbf{1}$ as a colourless solid $(85 \%, 369 \mathrm{mg})$.

Mp:. $285^{\circ} \mathrm{C}$ (decomp.).

${ }^{1}$ H-NMR (DMSO-d6, $400 \mathrm{MHz}, 25^{\circ} \mathrm{C}$ ): $\delta=7.96$ (s br, 2H), 7.59 (s br, 2H), 7.49 (s, 1H), $6.62(\mathrm{~s}, 2 \mathrm{H}), 3.73(\mathrm{~s}, 6 \mathrm{H}), 3.62(\mathrm{~s}, 3 \mathrm{H}), 3.59(\mathrm{~s}, 2 \mathrm{H})$.

${ }^{13}$ C-NMR (DMSO- $d 6,100 \mathrm{MHz}, 25{ }^{\circ} \mathrm{C}$ ): $\delta=163.9,154.5,152.8,139.8,136.1,133.1,108.8$, $106.3,59.9,55.9,32.1$.

IR (KBr): 3162 (w), 2724 (w), 1670 (m), 1640 (s), 1590 (m), 1499 (m), 1418 (m), 1341 (m), 1321 (m), 1238 (vs), 1129 (vs), 993 (m), 884 (m), 765 (m), 630 (m).

MS (70 eV, EI), m/z (\%): 290 (100, M+), 259 (21), 243 (10), 200 (4), 123 (5), 81 (2).

HRMS $m / z$ : calcd. for $\mathrm{C}_{14} \mathrm{H}_{18} \mathrm{~N}_{4} \mathrm{O}_{3}: 290.1379$; found: 290.1359 . 


\section{Trimethoprim 1}

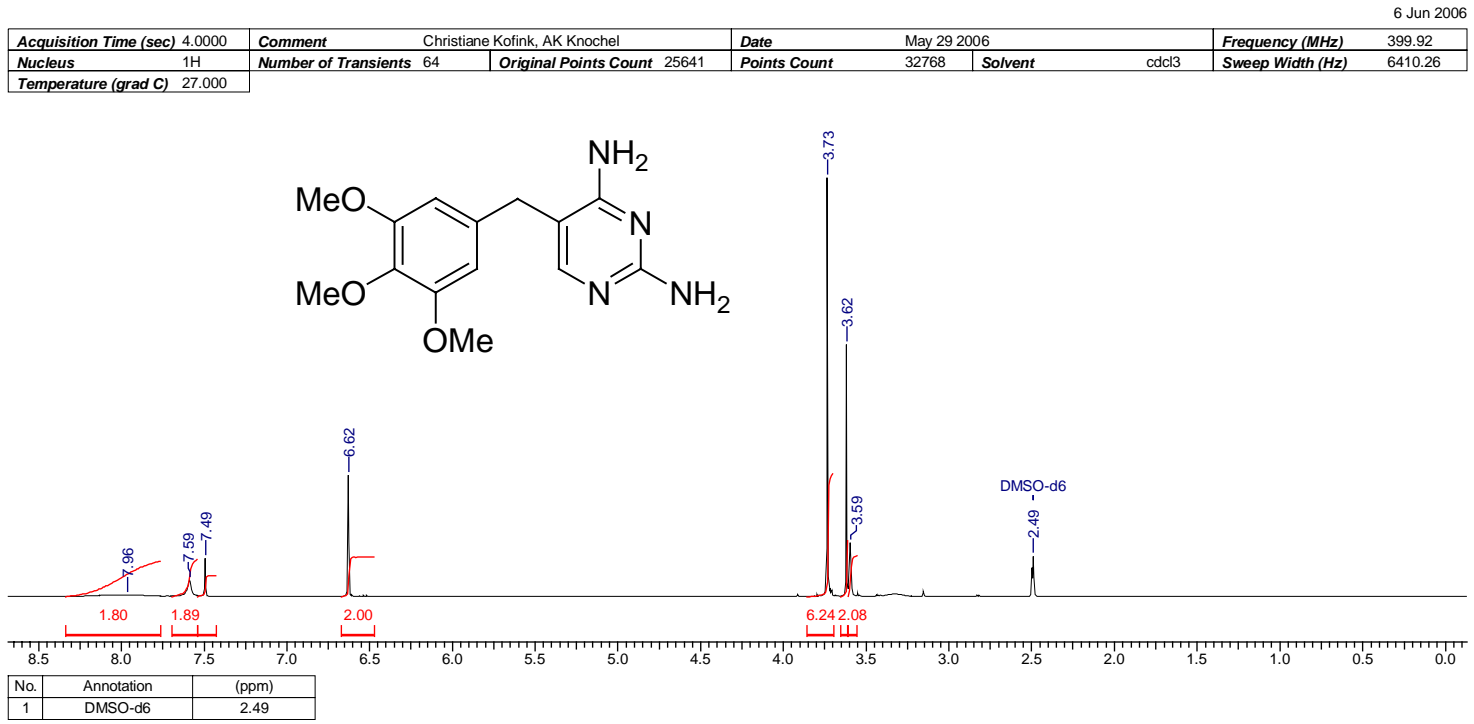

\section{Trimethoprim 1}

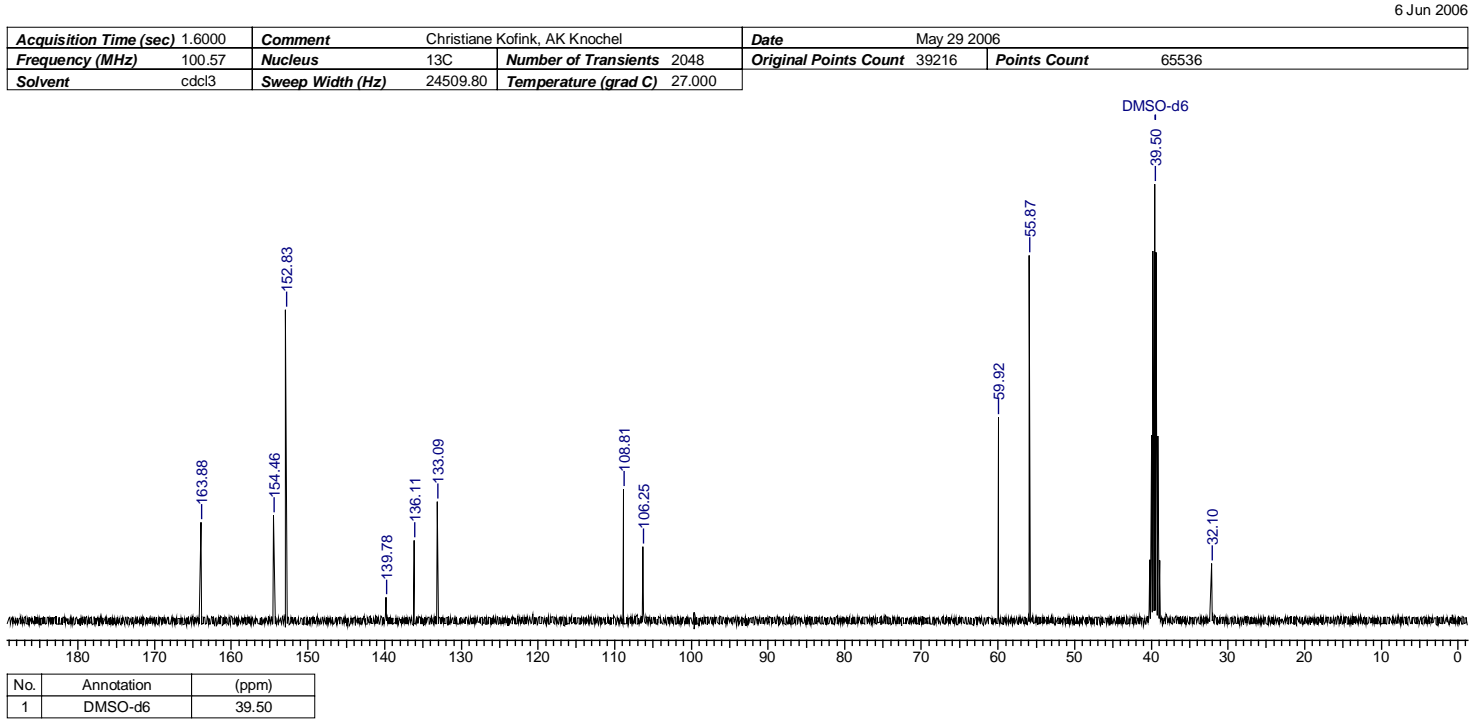

